\title{
Correlated Materials Design: Prospects and Challenges
}

\author{
Ran Adler ${ }^{1}$, Chang-Jong Kang ${ }^{1}$, Chuck-Hou Yee ${ }^{1}$, and Gabriel Kotliar ${ }^{1,2}$ \\ ${ }^{1}$ Dept. of Physics 85 Astronomy, Rutgers, The State University of New Jersey, Piscataway, NJ 08854, USA \\ ${ }^{2}$ Condensed Matter Physics and Materials Science Department, \\ Brookhaven National Laboratory, Upton, New York 11973, USA
}

(Dated: February 5, 2019)

\begin{abstract}
The design of correlated materials challenges researchers to combine the maturing, high throughput framework of DFT-based materials design with the rapidly-developing first-principles theory for correlated electron systems. We review the field of correlated materials, distinguishing two broad classes of correlation effects, static and dynamics, and describe methodologies to take them into account. We introduce a material design workflow, and illustrate it via examples in several materials classes, including superconductors, charge ordering materials and systems near an electronically driven metal to insulator transition, highlighting the interplay between theory and experiment with a view towards finding new materials. We review the statistical formulation of the errors of currently available methods to estimate formation energies. We formulate an approach for estimating a lowerbound for the probability of a new compound to form. Correlation effects have to be considered in all the material design steps. These include bridging between structure and property, obtaining the correct structure and predicting material stability. We introduce a post-processing strategy to take them into account.
\end{abstract}

\section{INTRODUCTION}

The ability to design new materials with desired properties is crucial to the development of new technology. The design of Silicon and Lithium-ion based materials are well known examples which led to the proliferation of consumer hand-held devices today. Materials discovery has historically proceeded via trial and error, with a mixture of serendipity and intuition being the most fruitful path. For example, all major classes of superconductors-from elemental Mercury in 1911, to the heavy Fermions, Cuprates and most recently, the ironbased superconductors-have been discovered largely by chance $^{1}$.

The dream of materials design is to create an effective workflow for discovering new materials by combining our theories of electronic structure, chemistry and computation. It is an inverse problem: start with the materials properties desired, and work to back out the chemical compositions and crystal structures which would lead to desirable properties. It requires a conceptual framework for thinking about the physical properties of materials, and sufficiently accurate methods for computing them. In addition it requires algorithms for predicting crystal structures and testing them for stability against decomposition, efficient codes implementing them and broadly accessible databases of known materials and their properties.

For weakly correlated materials, systems for which band theory works, significant progress in all these fronts has been made. Fermi liquid theory justifies our thinking of the excitations of a solid in terms of quasiparticles. Kohn Sham density functional theory (DFT) is a good tool for computing total energies and a good starting point for computing those quasiparticle properties in perturbation theory in the screened Coulomb interactions. Practical implementations of DFT such as LDA and GGA have become the underlying workhorse of the scientific community. Extensive benchmarks of software implementations $^{2}$ have shown that DFT reliably produces the total energy of a given configuration of atoms, enabling comparisons of stability between different chemical polymorphs. The maturity of DFT, combined with searchable repositories of experimental and calculated data (Materials Project ${ }^{3}$, OQMD ${ }^{4}$, AFLOWlib ${ }^{5}$ and NIMS $^{6}$ ), has fostered the growth of databases of computed materials properties to the point where one can successfully design materials (see for example Refs. 7-9).

Indeed these advances are beginning to pan out. The search for new topological materials such as topological insulators or Weyl semimetals is now greatly aided by electronic structure calculations (for a recent review see Ref. 10). Another clear example of this coming of age is the recent prediction of superconductivity in $\mathrm{H}_{3} \mathrm{~S}$ under high pressure near $190 \mathrm{~K}^{11}$. Subsequently, hydrogen sulfide was observed to superconduct near $200 \mathrm{~K}$, the highest temperature superconductor discovered so far ${ }^{12}$.

The situation is different for strongly correlated materials. Many aspects of the physics of correlated electron materials are still not well understood. Correlated systems exhibit novel phenomena not observed in weaklycorrelated materials: metal-insulator transitions, magnetic order and unconventional superconductivity are salient examples. Designing and optimizing materials with these properties would advance both technology and our understanding of the underlying physics.

Furthermore, material specific predictive theory for this class of materials is not fully developed, so even the direct problem of predicting properties of correlated materials with known atomic coordinates is very challenging. It requires going beyond perturbative approaches, and we currently lack methods for reliably modeling materials properties which scale up to the massive number of calculations necessary for material design purposes. 
In this article, we examine the challenges of material design projects involving strongly correlated electron systems. Our goal is to present the state of the art in the field stressing the outstanding challenges as it pertains to correlated materials, and propose strategies to solve them. We begin by providing a clear definition of correlations (Sec. II), distinguishing two important types, static and dynamic, and some available tools to treat them. Next we introduce the material design workflow (Sec. IV). Then we give five examples of materials design in correlated systems to illustrate the application of our ideas (Sec. VI A-VIE) and conclude with a brief outlook.

\section{WHAT ARE CORRELATED MATERIALS. STATIC AND DYNAMIC CORRELATIONS}

The standard model of periodic solids views the electrons in a crystal as freely propagating waves with well defined quantum numbers, crystal momentum and band index. Dating back to Sommerfeld and Bloch, it now has a firm foundation based on the Fermi liquid theory and the renormalization group, which explains why the effects of Coulomb interactions disappear or "renormalize away" at low energies, and provides an exact description of the excitation spectra in terms of quasiparticles. Another route to the band theory of solid, is provided by the density functional theory in the Kohn-Sham implementation, where a system of non-interacting quasiparticles is designed so as to provide the exact density of a solid. While this wave picture of a solid has been extraordinarily successful and is the foundation for the description of numerous materials, it fails dramatically for a class of materials, which we will denote strongly correlated electron systems.

The basic feature of correlated materials is their electrons cannot be described as non-interacting particles. Since the constituent electrons are strongly coupled to one another, studying the behavior of individual particles generally provides little insight into the macroscopic properties of a correlated material. Often, correlated materials arise when electrons are subjected to two competing tendencies: the kinetic energy of hopping between atomic orbitals promotes band behavior, while the potential energy of electron-electron repulsion prefers atomic behavior. When a system is tuned so that the two energy scales are comparable, neither the itinerant nor atomic viewpoint is sufficient to capture the physics. The most interesting phases generally occur in this correlated and difficult-to-describe regime, as we shall see in subsequent sections.

These ideas have to be sharpened in order to quantify correlation strength, as there is no sharp boundary between weakly and strongly correlated materials. Ultimately one would like to have a methodology which can explain the properties of any solid and which seeks to make predictions for comparison with experimental observations. To arrive at an operational definition of a correlated material, we examine DFT and how it relates to the observed electronic spectra.

The key idea behind DFT is that the free energy of a solid can be expressed as a functional of the electron density $\rho(\vec{r})$. Extremizing the free energy functional one obtains the electronic density of the solid, and the value of the functional at the extremum gives the total free energy of the material. The functional has the form $\Gamma[\rho]=\Gamma_{\text {univ }}[\rho]+\int d^{3} r V_{\text {cryst }}(r) \rho(r)$ where $\Gamma_{\text {univ }}[\rho]$ is the same for all materials, and the material-specific information is contained in the second term through the crystalline potential. The universal functional is written as a sum of $T[\rho]$ the kinetic energy, $E_{H}$, a Hartree Coulomb energy and a rest which is denoted as $F_{x c}$ the exchange correlation free energy. This term needs to be approximated since it is not exactly known, and the simplest approximation is to use the free energy of the electron gas at a given density. This is called the Local Density Approximation (LDA).

The extremization of the functional was recast by Kohn and Sham ${ }^{13}$ in the form of a single particle Schrödinger equation with the Hartree atomic units $\left(m_{e}=e=\hbar=\frac{1}{4 \pi \varepsilon_{0}}=1\right)$

$$
\begin{aligned}
{\left[-\frac{1}{2} \nabla^{2}+V_{K S}(\vec{r})\right] \psi_{\vec{k} j}(\vec{r}) } & =\epsilon_{\vec{k} j} \psi_{\vec{k} j}(\vec{r}) . \\
\sum_{\vec{k} j}\left|\psi_{\vec{k} j}(\vec{r})\right|^{2} f\left(\epsilon_{\vec{k} j}\right) & =\rho(\vec{r})
\end{aligned}
$$

reproduces the density of the solid. It is useful to divide the Kohn-Sham potential into several parts: $V_{K S}=$ $V_{H}+V_{c r y s t}+V_{x c}$, where one lumps into $V_{x c}$ exchange and correlation effects beyond Hartree. In practice, the exchange-correlation term is difficult to capture, and is generally modeled by approximations known as the local density approximation (LDA) or generalized gradient approximation (GGA). Density functional calculations using the LDA/GGA approximation have become very precise so that the uncertainties are almost entirely systematic. To get a feel for the numbers, convergence criteria of $10^{-1}$ to $10^{-4} \mathrm{meV} /$ atom are routinely used whereas differences between experimental and theoretical heats of formation routinely differ by over $100 \mathrm{meV} /$ atom $^{14}$.

The eigenvalues $\epsilon_{\vec{k} j}$ of the solution of the self-consistent set of Eqs. (1) and (2) are not to be interpreted as excitation energies. Konh Sham excitations are not Landau quasiparticle excitations. The latter represent the excitation spectra, which are the experimental observable in angle resolved photoemission and inverse photoemission experiments and should be extracted from the poles of the one particle Green's function:

$$
G\left(\vec{r}, \overrightarrow{r^{\prime}}, \omega\right)=\frac{1}{\omega+\frac{1}{2} \nabla^{2}+\mu-V_{H}-V_{\text {cryst }}-\Sigma\left(\vec{r}, \overrightarrow{r^{\prime}}, \omega\right)}
$$



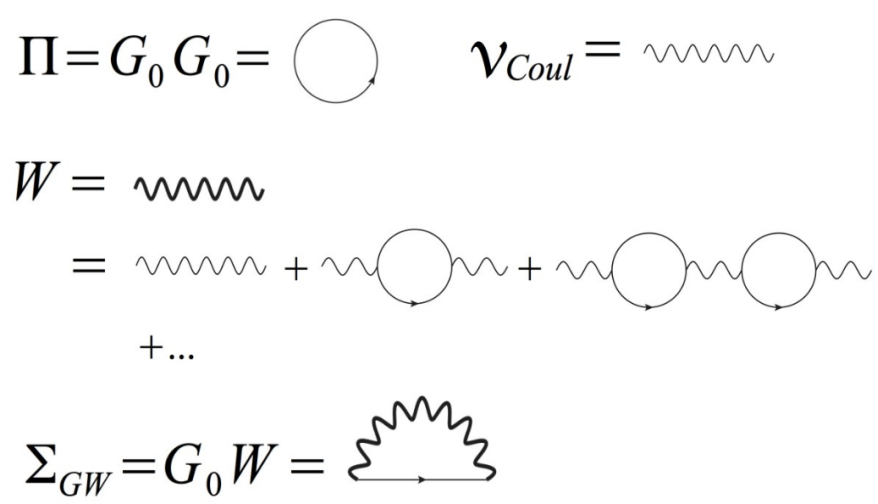

FIG. 1. Schematic diagrams for the GW method. Starting from some $G_{0}$ a polarization bubble is constructed, which is used to screen the Coulomb interactions resulting in an interaction $\mathrm{W}$. This $\mathrm{W}$ is then used to compute a self-energy $\Sigma_{G W}$ using W and $G_{0}$. To obtain the full Green's function $\mathrm{G}$ in Eq. (3), one goes from $\Sigma_{G W}$ to $\Sigma$ by subtracting the necessary single particle potential and uses the Dyson equation $G^{-1}=G_{0}^{-1}-\Sigma$ as discussed in the text. Adapted from 15 .

Here $\mu$ is the chemical potential and we have singled out in Eq. (3) the Hartree potential $V_{H}(\vec{r})=\int \frac{\rho\left(\vec{r}^{\prime}\right)}{\left|\vec{r}-\vec{r}^{\prime}\right|} d^{3} r^{\prime}$ expressed in terms of the exact density, $V_{\text {cryst }}$ is the crystal potential and we lumped the rest of the effects of the correlation in the self-energy operator $\Sigma\left(\vec{r}, r^{\prime}, \omega\right)$ which depends on frequency as well as on two space variables.

Since taking $\Sigma=V_{x c}$ generates the Kohn-Sham spectra, we define weakly correlated materials as ones where

$$
\left|\Sigma(\omega)-V_{\mathrm{xc}}\right|
$$

is small for low energies, so our definition of weakly correlated materials are those for which the Kohn-Sham spectra is sufficiently close to experimental results.

We can refine this definition, by taking into account first order perturbation theory in the screened Coulomb interactions, taking LDA as a starting point. This is the $G_{0} W_{0}$ method, which we now describe using diagrams in Fig. 1. This figure first describes the evaluation of the polarization bubble $\Pi$

$$
\Pi\left(t, t^{\prime}\right)=G_{0}\left(t, t^{\prime}\right) G_{0}\left(t^{\prime}, t\right)
$$

Next, the screened Coulomb potential $W$ in the random phase approximation (RPA) which is the infinite sum of diagrams depicted and represent the expression

$$
W^{-1}=v_{\text {Coul }}^{-1}-\Pi
$$

where $v_{\text {Coul }}$ is the bare Coulomb potential. Then one proceeds to the evaluation of a self-energy

$$
\Sigma_{G W}=G_{0} W
$$

which represents the lowest order contribution in perturbation theory in $\mathrm{W}$ (given in real space by Fig. 1), and then $G^{-1}=G_{0}^{-1}-\Sigma$ using Dyson's equation.

$G_{0}$ above is just a Green's function of non-interacting particles, and it can thus be defined in various ways, leading to different variants of the GW method. In the "oneshot" (that is, a method with no self-consistency loop) GW method (aka $G_{0} W_{0}$ ) one uses the LDA Kohn-Sham Green's function.

$$
G_{0}(i \omega)^{-1}=i \omega+\mu+\frac{1}{2} \nabla^{2}-V_{H}-V_{c r y s t}-V_{x c}^{L D A} .
$$

and the self-energy is thus taken to be $\Sigma=\Sigma_{G W}-V_{x c}^{L D A}$. Through this paper we use a matrix notation loosely and view operators as matrices. For example, in the Dyson equations $W, v_{\text {Coul }}, \Pi$ are operators (matrices) with matrix elements $\left\langle r|W(\omega)| r^{\prime}\right\rangle=W\left(\omega, r, r^{\prime}\right),\left\langle r|\Pi(\omega)| r^{\prime}\right\rangle=$ $\Pi\left(\omega, r, r^{\prime}\right),\left\langle r\left|v_{\text {Coul }}\right| r^{\prime}\right\rangle=\frac{1}{\left|r-r^{\prime}\right|}$.

This $G_{0} W_{0}$ method, introduced by Hybertsen and Louie $^{16}$, systematically improves the gaps of all semiconducting materials. We show this in Fig. 2. The success of this $G_{0} W_{0}$ method implies that in this kind of materials the Kohn-Sham references system is sufficiently close to the exact self-energy that the first order perturbation theory correction $\Sigma_{G_{0} W_{0}}(\omega)-V_{\mathrm{xc}}^{L D A / G G A}$ brings us close enough to the experimental results.

However, there are many materials (usually containing atoms with open $d$ or $f$ shells) where the photoemission spectra (and many other physical properties) are not so well described by this method. A successful many body theory of the solid state aims to describe all these systems. For the most widely used DFT starting points, LDA and GGA, what is the physical basis for the deviations in Eq. (4)? It is useful to think about two limiting cases, one in which the self-energy $\Sigma$ is a strong function of frequency, in which case we talk about dynamical effects, and a case where $\Sigma$ is strongly momentumdependent, or in real space - highly non-local, but weakly frequency dependent, and we talk about static correlations. In materials with strong dynamical correlations the spectral function displays additional peaks, which are not present in the band theory, and reflect the atomic multiplets of the material.

Electron correlation is customarily divided into dynamical and non-dynamical, but there is no strict definition of these terms. In the context of Quantum Chemistry calculations, these terms are mainly used to describe the ability of different methods to capture significant correlation effects, and the type of wave function which would approximate the exact solution of the Schrödinger equation. Non-dynamical or static correlations in the chemistry context means that energetically-close / degenerate electronic configurations are appreciably present in the wave function. This requires multiple Slater determinants of low lying configurations, and multi-reference methods to describe them, such as the multi-reference Hartree Fock method, or multi-reference coupled cluster methods. Dynamical correlation refers to a situation 


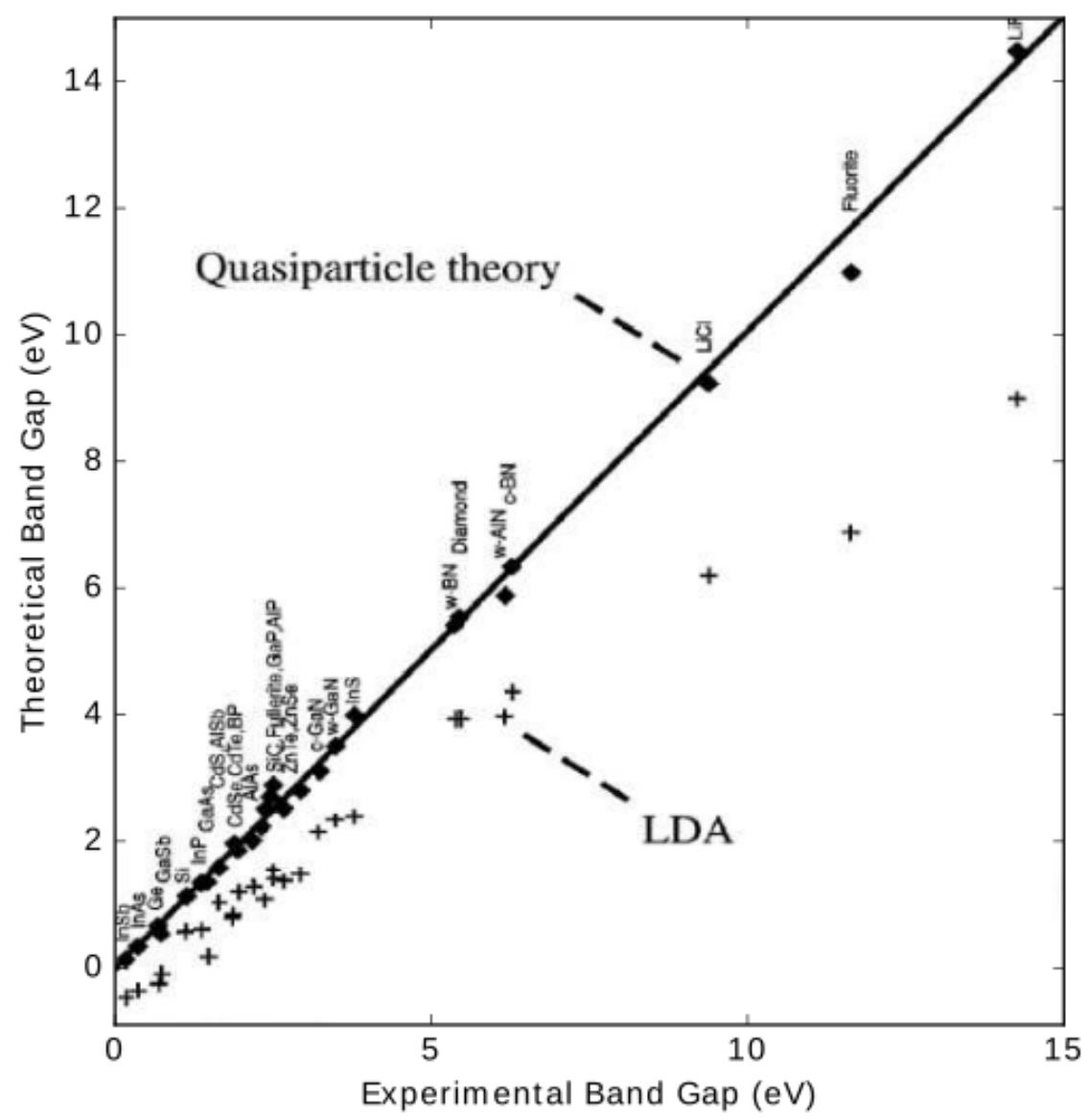

FIG. 2. Theoretically-determined semiconductor gap in a one shot LDA $G_{0} W_{0}$ calculation versus experiment (data complied by E. Shirley). Adapted from Chapter III. "First-principles theory of electron excitation energies in solids, surface, and defects" (article author: Steven G. Louie) in Topics in Computational Materials Science, edited by C. Y. Fong (World Scientific, 1998) [17]. Diamonds are the $G_{0} W_{0}$ excitation gap, while the crosses are the LDA value.

where a single Slater determinant, such as a closed shell configuration of some orbitals, is a good reference system - which then needs to be dressed by including double (or higher) excitations from strongly occupied core shells to empty orbitals. In addition, other virtual processes can modify the orbitals of the original slater determinant. This situation is well described in the standard coupled cluster method which is considered the gold standard in Quantum Chemistry ${ }^{18}$.

Confusingly, the chemist's delocalization error corresponds to our definition of $k$ dependent self-energy, which we denote as static correlation (since it does not involve frequency dependence of the self-energy), while the chemist's static correlation corresponds to what we call dynamical correlation as it requires a strongly frequency dependent self-energy in condensed matter physics. We use the solid state physicist convention in this article.

Another useful way to classify the correlations is by the level of locality of the self-energy. Introducing a complete basis set of localized wave functions labeled by site and orbital index we can expand the self-energy as

$$
\Sigma\left(\vec{r}, \vec{r}^{\prime}, \omega\right)=\sum_{\alpha \vec{R}, \beta \vec{R}^{\prime}} \chi_{\alpha \vec{R}}^{*}(\vec{r}) \Sigma_{\alpha \vec{R}, \beta \vec{R}^{\prime}}(\omega) \chi_{\beta \vec{R}^{\prime}}\left(\vec{r}^{\prime}\right) .
$$

The self-energy is approximately local when the onsite term $R=R^{\prime}$ in Eq. (9) is much larger than the rest. Notice that the notion of locality is defined with reference to a basis set of orbitals.

Equation (9) allows us to introduce an approximation to the self-energy ${ }^{19}$ involving a sum of a non-local but frequency independent term plus a frequency dependent but local self-energy:

$$
\Sigma(\vec{k}, \omega) \simeq \Sigma(\vec{k})+\sum_{\vec{R}, \alpha \beta \in L}|\vec{R} \alpha\rangle \Sigma_{\alpha \overrightarrow{R, \beta \vec{R}}}(\omega)\langle\vec{R} \beta|
$$

This ansatz was first introduced by Sadovskii $e t a l^{20}$. It is useful when the sum over orbitals in Eq. (10) runs over a 


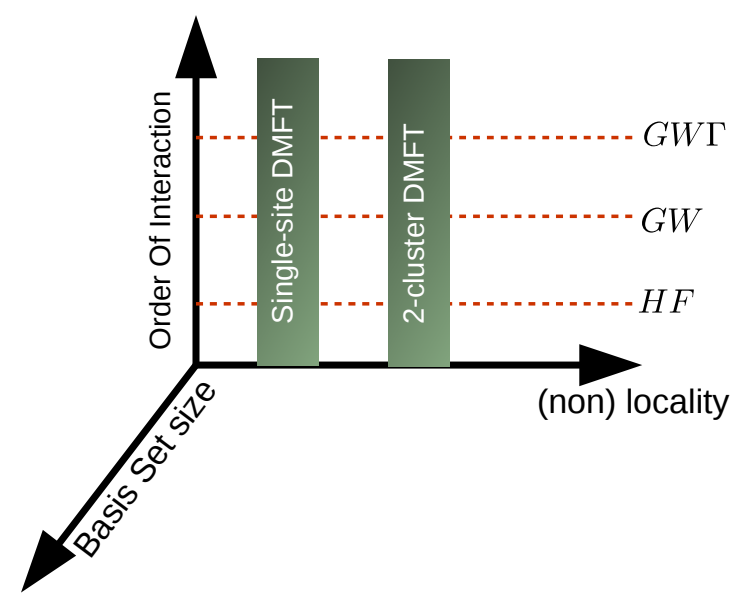

FIG. 3. Two complementary approaches to the treatment of correlations. One axis represents the systematic perturbative expansion in powers of an interaction (for example the screened Coulomb interaction W). The second axis, sums perturbation theory to all orders, but at the local level. When locality is just a lattice site, we have the single site DMFT, improvements involve larger clusters. In addition when one goes beyond model Hamiltonians towards the realistic treatment of solids we need to introduce a basis set and estimate that the results are converged as function of the size of the basis set.

small set $L$ (much smaller than the size of the basis set), for example over a single shell of $d$ or $f$ orbitals. This form captures both static and dynamical correlations and is also amenable to computation using Dynamical Mean Field Methods to be introduced in section III.

\section{HOW TO TREAT CORRELATIONS}

Having defined correlations as a departure of the Green's function from the results of lowest order perturbation theory around LDA (i.e. $G_{0} W_{0}$ ), we now review various ways to correlations into account. One should keep in mind that different materials may require stronger momentum or frequency dependence in their self-energy, and may exhibit different degrees of locality. This section lays out several complementary approaches to treat correlations beyond $G_{0} W_{0}$. They represent different compromises between speed and accuracy, and can target different levels of locality and different correlations strengths. A schematic view of the grand challenge posed by the treatment of correlations in the solid state is presented in Fig. 3, which explains the need to converge the calculations along multiple axis.

Linearized Self Consistent Quasiparticle GW. We begin our treatment with the GW approximation, which was introduced in the previous section. One obvious flaw of the $G_{0} W_{0}$ method is its dependence on the LDA input. This makes the method increasingly inaccurate as the strength of the correlations increase. One way to eliminate this dependence, is to introduce some level of self consistency. Hedin ${ }^{21}$ proposed a full self-consistent $G W$ scheme, namely to use $G_{0}=G$ is in Eq. (7). We can think of this as setting $V_{x c}=0$, so it is not used in intermediate steps. There are numerous advantages, however, to using a non-interacting form for $G_{0}$ in the algorithm, and in practice the spectra in self-consistent GW turned out to be consistently worse in solids than the non-selfconsistent approach for spectral properties ${ }^{22}$. Nevertheless, GW can be reasonably accurate for total energy calculations, as they can be obtained as stationary points of a functional ${ }^{23,24}$.

To improve on the spectra relative to $G_{0} W_{0}$ while retaining some level of self consistency so as not to depend on the starting point, the self-consistent quasi-particle GW (QPGW) $)^{25}$ was proposed. Here one uses the "best" non-interacting Green's function $G_{0}$, which is defined in terms of an "exchange and correlation potential" $V_{x c}^{Q P G W}$ chosen to reproduce the spectra of the full G as closely as possible:

$G_{0}^{Q P G W}(i \omega)^{-1}=i \omega+\mu+\frac{1}{2} \nabla^{2}-V_{H}-V_{\text {cryst }}-V_{x c}^{Q P G W}$.

To determine $V_{x c}^{Q P G W}$ (which once again we view as a matrix with matrix elements $\left\langle r\left|V_{x c}^{Q P G W}\right| r^{\prime}\right\rangle=$ $\left.V_{x c}^{Q P G W}(r) \delta\left(r-r^{\prime}\right)\right)$, it was proposed to approximate the spectra and the eigenvectors of $\mathrm{G}$ by those of $G_{0}^{Q P G W}$ - by solving a set of non-linear equations on the real axis ${ }^{25}$. An alternative approach that works on the imaginary axis is to linearize the GW self-energy at each iteration. Namely, after the evaluation of the self-energy in Eq. (7), this quantity is Taylor expanded around zero frequency (hence the name "linearized"):

$$
\Sigma_{l i n}(\vec{k}, i \omega)=i \omega\left(1-Z(\vec{k})^{-1}\right)+\Sigma(\vec{k}, 0)
$$

and $G_{0}^{Q P G W}(i \omega)$ is obtained by solving the usual Dyson equation with the linearized self-energy, and multiplying the result by the quasiparticle residue, $Z$, to obtain a properly normalized quasiparticle Green's function.

$$
\begin{aligned}
& G_{0}^{\mathrm{QPGW}}= \\
& \sqrt{Z(\vec{k})}\left[i \omega+\mu+\frac{1}{2} \nabla^{2}-V_{H}-V_{\text {cryst }}-\Sigma_{\text {lin }}\right]^{-1} \sqrt{Z(\vec{k})}
\end{aligned}
$$

Note that this defines the exchange correlation potential of the self-consistent QPGW method. This method, the linearized self-consistent quasiparticle GW, was introduced in Ref. 26 and an open source code to implement this type of calculation in the linearized augmented plane wave (LAPW) basis set is available in Ref. 27.

The GW or RPA method captures an important physical effect. Electrons are charged objects which interact via the long range Coulomb interactions. Quasiparticles, on the other hand, interact through the screened Coulomb interaction. They are composed of electrons surrounded by screening charges, thus reducing the 


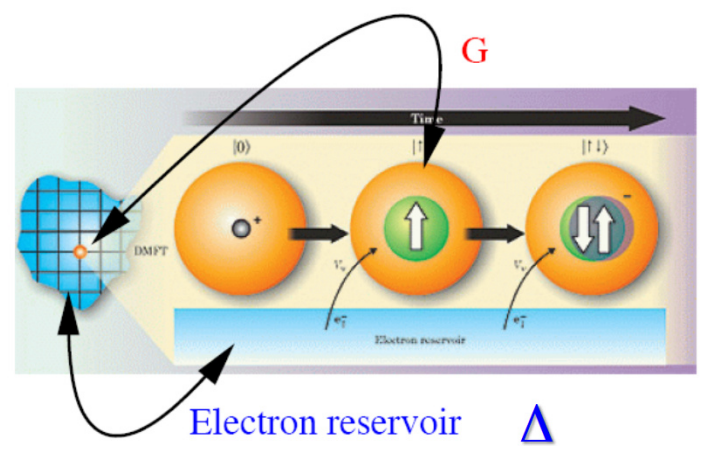

FIG. 4. Dynamical Mean Field Theory (DMFT) maps (or truncates) a lattice model to a single site embedded in a medium (impurity model) with a hybridization strength which is determined self consistently. Adapted from Ref. 28.

strength and the range of their interaction. For this reason, in many model Hamiltonians describing metals, only the short range repulsion is kept. On the other hand, it is well known that the RPA fails in describing the pair correlation function at short distances. One can say that the GW method captures the long range of the screening effects of the long range Coulomb interactions and produce a self-energy which is non-local in space, but with a weak frequency dependence (indeed the self-energy is linear in a broad range of energies). It turns out that this method is not able to capture the effects of the short range part of the Coulomb interactions which in turn induces strong frequency dependence (i.e. strong non-locality in time), but in turn is much more local in space.

Dynamical Mean Field Theory (DMFT). To capture dynamical local correlations one uses Dynamical mean field theory ${ }^{29}$, which is the natural extension of the Weiss mean field theory of spin systems to treat quantum mechanical model Hamiltonians. Dynamical Mean Field Theory becomes exact in the limit of infinite dimensions, which was introduced by Metzner and Vollhardt ${ }^{30}$. With suitable extensions it plays an important role in realistically describing strongly correlated electron materials. Here we describe the main intuitive DMFT ideas as a quantum embedding, starting from the example of a oneband Hubbard model (describing $s$ electrons), in which the relevant atomic configurations are $|0\rangle,|\uparrow\rangle,|\downarrow\rangle,|\uparrow \downarrow\rangle$ as described in Fig. 4. It involves two steps. The first step, focuses on a single lattice site, and describes the rest of the sites by a medium with which an electron at this site hybridizes. This truncation to a single site problem is common to all mean field theories. In the Weiss mean field theory one selects a spin at a given site, and replaces the rest of the lattice by an effective magnetic field or Weiss field. In the dynamical mean field theory, the local Hamiltonian at a given site is kept, and the kinetic energy is replaced by a hybridization term with a bath of non-interacting electrons, which allows the atom at the selected site to change its configuration. This is depicted in Fig. 4 where we apply the method to the one-band Hubbard model. The system consist of one band of $s$

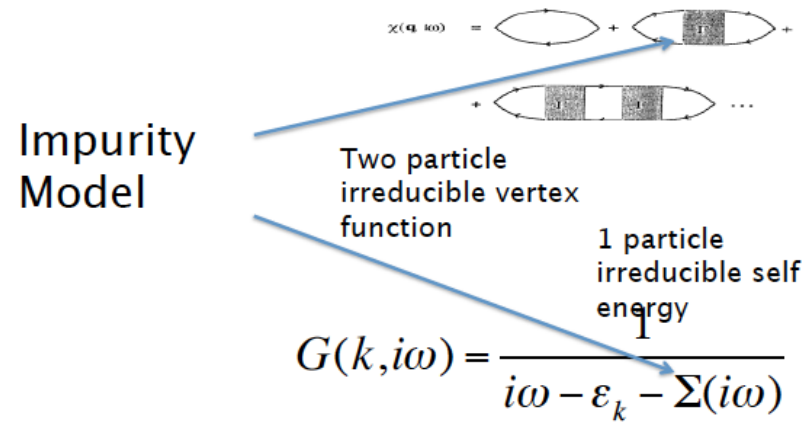

FIG. 5. The DMFT impurity model is used to generate irreducible quantities such as self-energies and one particle vertices. These are then embedded in the lattice model to generate momentum dependent lattice quantities such as spectral functions, or spin susceptibilities. Adapted from 15 .

electrons. The Fourier transform of the hopping integral is given by $t(\vec{k})$.

It is used in the second step, which involves the reconstruction of lattice observables by embedding the local impurity self-energy into a correlation function of the lattice,

$$
G_{l a t t}(\vec{k}, i \omega)^{-1}=i \omega+\mu-t(\vec{k})-\Sigma_{i m p}(i \omega)
$$

Here $\Sigma_{i m p}(i \omega)$ are viewed as functionals of the Weiss field. The requirement that $\sum_{k} G_{l a t t}=G_{l o c}$ determines the Weiss field. Table I summarizes the analogies between Weiss mean field theory and dynamical mean field theory.

\begin{tabular}{l|l}
\hline Weiss Mean Field Theory & Dynamical Mean Field Theory \\
\hline $\begin{array}{l}\text { Ising Model } \rightarrow \text { Single Spin } \\
\text { in effective Weiss Field }\end{array}$ & $\begin{array}{l}\text { Hubbard Model } \rightarrow \\
\text { Impurity in effective bath }\end{array}$ \\
\hline Weiss field: $h_{e f f}$ & effective bath: $\Delta\left(\imath \omega_{n}\right)$ \\
\hline Local observable: $m=<s_{i}>$ & Local Observable: $G_{l o c}\left(\imath \omega_{n}\right)$ \\
\hline Self-consistent condition: & Self-consistent condition: \\
$\tanh \left(\beta \sum_{j} J_{i j} s_{j}\right)=m$ & $i \omega_{n}-E_{i m p}-\Delta\left(i \omega_{n}\right)$ \\
& $-\Sigma\left(i \omega_{n}\right)=\left[\sum_{\vec{k}} G_{\vec{k}}\left(i \omega_{n}\right)\right]^{-1}$ \\
\hline
\end{tabular}

TABLE I. Corresponding quantities in Dynamical MFT (right) and Weiss or static MFT in statistical mechanics (left).

The DMFT mapping of a lattice model into an impurity model gives a local picture of the solid, in terms of an impurity model, which can then be used to generate lattice quantities such as the electron Green's function and the magnetic susceptibility by computing the corresponding irreducible quantities. This is illustrated in Fig. 5.

The self-consistent loop of DMFT is summarized in the following iterative cycle 


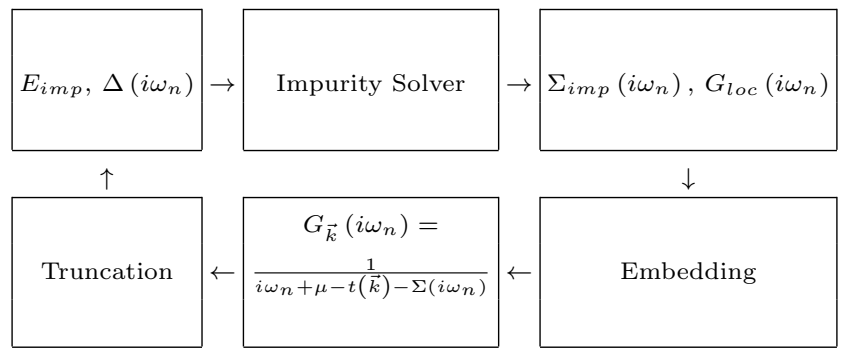

From the simplest model, the one-band Hubbard model, one can proceed to more realistic descriptions of correlated materials by replacing $t(\vec{k})$ by a tight-binding model Hamiltonian matrix. The DMFT equations can be derived from a functional

$$
\begin{aligned}
& \Gamma_{D M F T_{\text {model }}}\left[G_{\alpha \beta, \vec{R}}, \Sigma_{\alpha \beta, \vec{R}}\right]= \\
& -\operatorname{Tr} \ln \left[i \omega_{n}-H(\vec{k})-\Sigma_{\alpha \beta \vec{R}}\left(i \omega_{n}\right)\right] \\
& -\sum_{n} \operatorname{Tr}\left[\Sigma\left(i \omega_{n}\right) G\left(i \omega_{n}\right)\right]+\sum_{\vec{R}} \Phi\left[G_{\alpha \beta, \vec{R}}, U\right]
\end{aligned}
$$

where $\Phi\left[G_{\alpha \beta, \vec{R}}, U\right]$ is the Baym-Kadanoff functional the sum of all two particle irreducible diagrams in terms of the full Green's function $G$, and the Hubbard interaction $U$, which denotes a four rank tensor $U_{\alpha \beta \gamma \delta}$. It can also be evaluated from the Anderson impurity model expressed in terms of the full local Green's function of the impurity $G$. The impurity model is the engine of a DMFT calculation. Multiple approaches have been used for its solution, and full reviews have been written on the topic. The introduction of continuous time Monte Carlo method for impurity models ${ }^{31}$ have provided numerically exact solutions reducing the computational cost relative to the Hirsch-Fye algorithm that was used in earlier DMFT studies.

DFT+DMFT method. This is the next step towards a more realistic description of solids. It was introduced in Refs. 32 and 33. In these early implementations, it consisted of replacing the Hamiltonian $H(\vec{k})$ by the Kohn-Sham matrix in Eq. (13) with a correction to subtract the correlation energy that is contained in the Kohn-Sham Hamiltonian (double counting correction). The original DFT calculations were carried out with an LDA exchange and correlation potential, but they could be done with GGA and other functionals. Furthermore the exchange and correlation potential in the Dyson equation for the Green's function can be replaced by another static mean field theory like hybrid DFT or QPGW, but in the following we will use the terminology LDA+DMFT.

Starting from the Anderson model Hamiltonian point of view, one divides the orbitals into two sets. The first set contains the large majority of the electrons are prop- erly described by the LDA Kohn-Sham matrix. The second set contains the more localized orbitals ( $d$-electrons in transition metals and $f$-electrons in rare earths and actinides) which require the addition of DMFT corrections. A subtraction (called the double counting corrections) takes into account that the Hartree and exchange correlation has been included in that orbital twice, since it was treated both in LDA and in DMFT. The early LDA+DMFT calculations, proceeded in two steps (oneshot LDA+DMFT). First an LDA calculation was performed for a given material. Then a model Hamiltonian was constructed from the resulting Kohn-Sham matrix corrected by $E_{d c}$ written in a localized basis set. The values of a Coulomb matrix for the correlated orbitals were estimated or used as fitting parameters. Finally DMFT calculation were performed to improve on the one particle Green's function of the solid.

In reality, the charge is also corrected by the DMFT self-energy, which in turn changes the exchange and correlation potential away from its LDA value. Therefore charge self-consistent LDA+DMFT is needed. This was first implemented in Refs. 34 and 35.

For this purpose it is useful to notice that the LDA+DMFT equations can be derived as stationary points of an LDA+DMFT functional, which can be viewed as a functional of the density and local Green's function of correlated orbitals. This is a spectral density functional. Evaluating the functional at the stationary point gives the free energy of the solid, and the stationary Green's functions gives us the spectral function of the material. We can arrive at the DFT + DMFT functional by performing the substitutions $-\frac{1}{2} \nabla^{2}+V_{K S}(\vec{r})$ for $H(\vec{k})$ in the model DMFT functional Eq. (13) and then adding terms arising from the density functional theory, namely:

$$
\begin{aligned}
& \Gamma_{D F T+D M F T}\left[\rho(\vec{r}), G_{\alpha \beta, \vec{R}}, V_{K S}(\vec{r}), \Sigma_{\alpha \beta, \vec{R}}\right] \\
& =\Gamma_{D M F T_{\text {model }}}\left[H(\vec{k}) \rightarrow-\frac{1}{2} \nabla^{2}+V_{K S}(\vec{r})\right] \\
& +\Gamma_{2}\left[V_{K S}(\vec{r}), \rho(\vec{r})\right]-\Phi_{D C}
\end{aligned}
$$

where

$$
\begin{aligned}
\Gamma_{2}\left[V_{K S}(\vec{r}), \rho(\vec{r})\right] & =-\int V_{K S}(\vec{r}) \rho(\vec{r}) d^{3} r \\
& +\int V_{\text {ext }}(\vec{r}) \rho(\vec{r}) d^{3} r \\
& +\frac{1}{2} \int \frac{\rho(\vec{r}) \rho\left(\vec{r}^{\prime}\right)}{\left|\vec{r}-\vec{r}^{\prime}\right|} d^{3} r d^{3} r^{\prime}+E_{x c}^{D F T}[\rho]
\end{aligned}
$$

We then arrive at the DFT+DMFT functional which we write in full below.

$$
\Gamma_{D F T+D M F T}\left[\rho(\vec{r}), G_{\alpha \beta, \vec{R}}, V_{K S}(\vec{r}), \Sigma_{\alpha \beta, \vec{R}}\right]=
$$




$$
\begin{aligned}
& -\operatorname{Tr} \ln \left[i \omega_{n}+\mu+\frac{\nabla^{2}}{2}-V_{K S}-\sum_{R, \alpha \beta \in L} \chi_{\alpha \vec{R}}^{*}(\vec{r}) \Sigma_{\alpha \beta \vec{R}}\left(i \omega_{n}\right) \chi_{\beta \vec{R}}\left(\vec{r}^{\prime}\right)\right] \\
& -\int V_{K S}(\vec{r}) \rho(\vec{r}) d^{3} r-\sum_{n} \operatorname{Tr}\left[\Sigma\left(i \omega_{n}\right) G\left(i \omega_{n}\right)\right]+\int V_{c r y s t}(\vec{r}) \rho(\vec{r}) d^{3} r \\
& +\frac{1}{2} \int \frac{\rho(\vec{r}) \rho\left(\vec{r}^{\prime}\right)}{\left|\vec{r}-\vec{r}^{\prime}\right|} d^{3} r d^{3} r^{\prime}+E_{x c}^{D F T}[\rho]+\sum_{\vec{R}} \Phi\left[G_{\alpha \beta, \vec{R}}, U\right]-\Phi_{D C} .
\end{aligned}
$$

$\Phi$ is the sum of two-particle irreducible diagrams written in terms of $G$ and $U$. It was written down first in Ref. 34, building on the earlier work of Chitra and Kotliar 36,37 . It is essential for total energy calculations which require the implementation of charge self-consistency in the LDA+DMFT method. The first implementation of charge self-consistent LDA+DMFT was carried out in a full potential linear muffin-tin orbital (FP-LMTO) basis set $^{34}$. It was used to compute total energy and phonons of $\delta$-plutonium ${ }^{35,38}$.

Alternatively, one can include the hybridization function $\Delta$ or the Weiss field $\mathcal{G}$ as an independent variable in the functional in order to see explicitly the free energy of the Anderson Impurity Model, $\mathcal{G}_{\alpha \beta, \vec{R}}^{-1}=G_{\text {atom }{ }_{\alpha, \beta, \vec{R}}}^{-1}$
$\Delta_{\alpha \beta, \vec{R}}$

$$
F_{i m p}\left[\mathcal{G}_{\alpha \beta, \vec{R}}^{-1}\right]=-\ln \int D\left[c^{\dagger} c\right] e^{-S_{i m p}\left[c^{\dagger}, c\right]}
$$

with

$$
\begin{array}{r}
S_{i m p}\left[\mathcal{G}_{\alpha \beta, \vec{R}}^{-1}\right]=-\sum_{\alpha \beta} \int d \tau d \tau^{\prime} c_{\alpha}^{\dagger}(\tau) \mathcal{G}_{\alpha \beta, \vec{R}}^{-1}\left(\tau, \tau^{\prime}\right) c_{\beta}\left(\tau^{\prime}\right) \\
+U_{\alpha \beta \gamma \delta} \int d \tau c_{\alpha}^{\dagger}(\tau) c_{\beta}^{\dagger}(\tau) c_{\delta}(\tau) c_{\gamma}(\tau)
\end{array}
$$

So that:

$$
\begin{aligned}
& \Gamma_{D F T+D M F T}\left[\rho(\vec{r}), G_{\alpha \beta, \vec{R}}, V_{K S}(\vec{r}), \Sigma_{\alpha \beta, \vec{R}},{ }_{\alpha \beta, \vec{R}}, \mathcal{G}_{\alpha \beta, \vec{R}}\right]= \\
& -\operatorname{Tr} \ln \left[i \omega_{n}+\mu+\frac{\nabla^{2}}{2}-V_{K S}-\sum_{R, \alpha \beta \in L} \chi_{\alpha \vec{R}}^{*}(\vec{r}) \Sigma_{\alpha \beta \vec{R}}\left(i \omega_{n}\right) \chi_{\beta \vec{R}}\left(\vec{r}^{\prime}\right)\right] \\
& -\int V_{K S}(\vec{r}) \rho(\vec{r}) d^{3} r-\sum \operatorname{Tr}\left[\left(\mathcal{G}^{-1}-\Sigma\left(i \omega_{n}\right)-G^{-1}\right) G\left(i \omega_{n}\right)\right]+\int V_{c r y s t}(\vec{r}) \rho(\vec{r}) d^{3} r \\
& +\frac{1}{2} \int \frac{\rho(\vec{r}) \rho\left(\vec{r}^{\prime}\right)}{\left|\vec{r}-\vec{r}^{\prime}\right|} d^{3} r d^{3} r^{\prime}+E_{x c}^{D F T}[\rho]+\sum_{\vec{R}} F_{i m p}\left[\mathcal{G}_{\alpha \beta, \vec{R}}^{-1}\right]-\operatorname{Tr} \ln \left[G_{\alpha \beta, \vec{R}}\right]-\Phi_{D C} .
\end{aligned}
$$

The form of the LDA+DMFT functional makes it clear that the method is independent of the basis set used to implement the electronic structure calculation, provided that the basis is complete enough. On the other hand, it is clearly dependent on the parameter $U$ chosen, on the form of the double counting correction and the choice of the projector (i.e., the orbitals $\chi_{\alpha}(\vec{r})$ with $\alpha \in L$ that enter this definition) and the exchange correlation functional $E_{x c}^{D F T}$. A projector of the form $P\left(r, r^{\prime}\right)=\sum_{\alpha \beta \in L} \chi_{\alpha \vec{R}}^{*}(\vec{r}) \chi_{\beta} \vec{R}\left(\vec{r}^{\prime}\right)$ was used to define a truncation from $G$ to $G_{l o c}$. The inverse of $P$ is the embedding operator $E$ defined by $P \cdot E=I_{L}$ where $I_{L}$ is the identity operator in the space spanned by the correlated orbitals. If one restricts $E \cdot P$ to the space $L$, one also obtains the identity operator in that space. $E$ is used to define an embedding of the self-energy $\Sigma\left(r, r^{\prime}\right)=\sum_{\alpha \beta} E^{\alpha, \beta}\left(r, r^{\prime}\right) \Sigma_{\alpha, \beta}^{l o c}$.
However, more general projectors can be considered as long as causality of the DMFT equations is satisfied. Ideas for choosing an optimal projector for LDA+DMFT based on orbitals were presented in Ref. 39. Choosing suitable projectors (and correspondingly a suitable value of the $U$ matrix and a proper double counting correction) is crucial for the accuracy of an LDA+DMFT calculation as demonstrated recently in the context of the hydrogen molecule ${ }^{40}$. DFT+DMFT is now a widely used method. It has been successfully used across the periodic table, and has been implemented in numerous codes ${ }^{41-50}$. Still there is ample room for advances in implementation, and on providing a firm foundation of the method. One can view the DFT+DMFT functional written above, as an approximation to an exact DFT+DMFT functional, which would yield the exact density and spectra of the solid $^{37}$. This viewpoint has been used recently to provide 

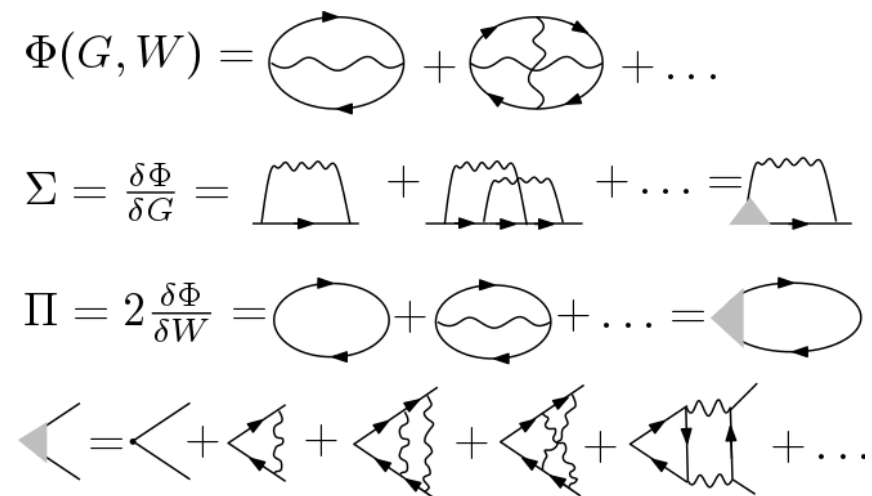

FIG. 6. Hedin's Equations give an exact representation of the correlation function of the Bosonic and Fermionic correlation in an expansion in $\mathrm{G}$ and W. They can be obtained by setting the functional derivatives of the Eq. (18) to $0 . \quad \Phi(G, W)$ (first line) is the set of 2-PI skeleton diagrams (in G and $\mathrm{W}$ ), where by convention the symmetry weights are omitted. The derivative by $\mathrm{G}$ (line 2 ) shows how the self-energy $\Sigma$ is defined in terms of a 3-legged vertex $\Gamma$. The derivative by $W$ (line 3 ) equals the polarization $\Pi$. The bottom line shows the definition of the vertex $\Gamma$.

an expression for the double counting correction $\Phi_{D C}{ }^{51}$. An alternative perspective goes back to a fully diagrammatic many body theory of the solid, and examines how DFT+DMFT would fit in that framework as an approximation. We turn to this formulation next.

Fully Diagrammatic Methods: The free energy of the solid can also be expressed as a functional of $G\left(x, x^{\prime}\right)$ and $W\left(x, x^{\prime}\right)$ by means of a Legendre transformation and results in Refs. 36 and 52, where $E_{H}=$ $\frac{1}{2} \int \frac{\rho(\vec{r}) \rho\left(\vec{r}^{\prime}\right)}{\left|\vec{r}-\vec{r}^{\prime}\right|} d^{3} r d^{3} r^{\prime}, \Phi$ is defined as sum of all 2-particle irreducible diagrams which cannot be divided into two parts by cutting two Green's functions lines (which can be either G's or W's):

$$
\begin{aligned}
\Gamma[G, W, \Sigma, \Pi] & =-\operatorname{Tr} \ln \left[G_{0}^{-1}-\Sigma\right]-\operatorname{Tr}[\Sigma G] \\
& +\frac{1}{2} \operatorname{Tr} \ln \left[v_{\text {Coul }}^{-1}-\Pi\right]-\frac{1}{2} \operatorname{Tr}[\Pi W] \\
& +E_{H}+\Phi[G, W],
\end{aligned}
$$

This reformulation is exact and leads to the exact Hedin's equations, shown in Fig. 6. To convert this general method into a tool of practical use, strong approximations have to be introduced.

The lowest order graphs of Eq. (18), shown in Fig. 7, reproduce the self-consistent GW approximation: taking functional derivatives of the low order functional with respect to the arguments produces the same equations as the GW approximation.

To summarize the discussion so far, we recall that for semiconductors, non-local (but weakly frequency dependent) correlation effects are needed to increase the gap from its LDA value. This admixture of exchange, can be done within the GW method, or using hybrid density functionals. It reflects the importance of exchange beyond the LDA, which is due to the long-range but static

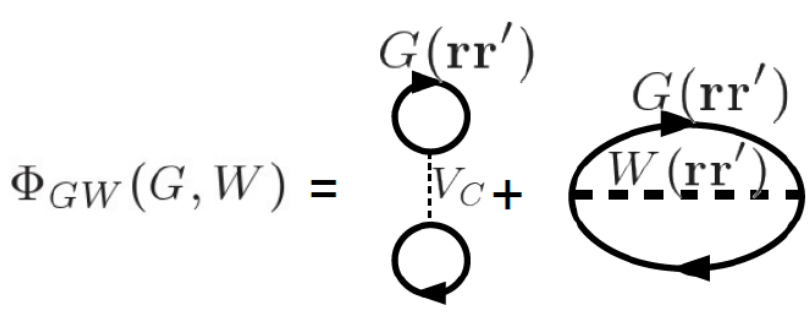

FIG. 7. Lowest order graphs in the $\Phi$-functional of Eq. (18). They give rise to the fully self-consistent GW approximation, as the saddle point equations. Note that the first term here is the Hartree energy. Adapted from 15.

part of the Coulomb interaction. These are the static correlation effects. It has recently been shown, that this type of correlation effects are important in materials near a metal-to-insulator transition such as $\mathrm{BaBiO}_{3}$ or $\mathrm{HfClN}^{53}$ and can have a dramatic effect in enhancing the electron-phonon interaction relative to its LDA estimated value. In these systems, a strongly k-dependent self-energy effect, $\Sigma(k)$, is much more important than frequency dependence, and here GW methods work well.

On the other hand frequency dependence, and its implied non-locality in time, is crucial to capture Mott or Hund's physics. This physics tends to be local in space and can be captured by DMFT. Static mean field theories, such as the LDA, do not capture this non-locality in time, and therefore fail to describe Mott or Hund's phenomena. DFT+DMFT can treat strong frequency dependency, but has k-dependence only as inherited from the k-dependence of DFT exchange and correlation potential, the k-dependence of the embedding and the double counting shift.

In real materials both effects are present to some degree - thus motivating physically the ansatz, Eq. (10). Some examples discussed recently are $\mathrm{Ce}_{2} \mathrm{O}_{3}$ (using hybrid DFT+DMFT) in Ref. 54 and the iron pnictides and chalcogenides in Ref. 19.

We now describe a route proposed by Chitra ${ }^{36,37}$ to embed DMFT into a many-body approach to electronic structure within a purely diagrammatic approach formulated in the continuum.

If one selects a projector, which allows us to define a local Green's function, it was suggested in Refs. 36, 37 , and 55 that one can perform a local approximation and keep only the local higher order graphs in a selected orbital.

$$
\begin{aligned}
& \Phi[G, W] \simeq \\
& \Phi_{E D M F T}\left[G_{l o c}, W_{l o c}, G_{\text {nonlocal }}=0, W_{\text {nonlocal }}=0\right]+ \\
& \Phi_{G W}-\Phi_{G W}\left[G_{l o c}, W_{l o c}, G_{\text {nonlocal }}=0, W_{\text {nonlocal }}=0\right]
\end{aligned}
$$

Since the lowest-order graph is contained in the GW approximation, one should start from the second order graph and higher order. This $\Phi_{G W+D M F T}$ functional is shown in Fig. 8.

These ideas were formulated and fully implemented in the context of a simple extended Hubbard model ${ }^{57,58}$. 


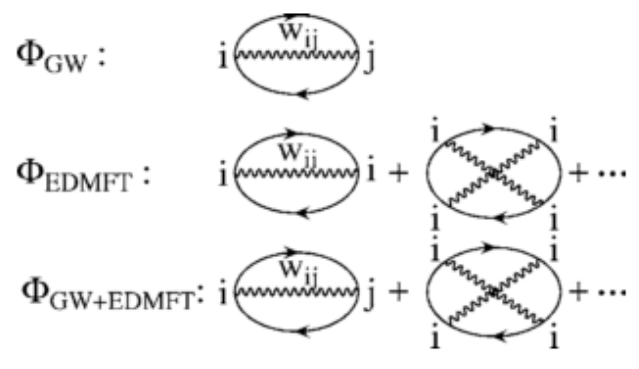

FIG. 8. Comparison of the functionals for the methods described in the text. The Hartree diagram was dropped since it appears in all methods ${ }^{56}$.

An open problem in this area, explored in Ref. 58 is the level of self consistency that should be imposed. This important issue is already present in the implementation of the GW method, and the work of Ref. 58 should be revisited using the lessons from the QPGW method ${ }^{19}$. There has been a large number of works exploring GW+DMFT and related extensions and combinations, and we refer the reader to recent reviews for the most recent references $^{59}$. Recently, we proposed the self consistent quasiparticle GW+DMFT ${ }^{60,61}$, as a theory that contains both the most successful form of a GW approximation and DMFT as limiting cases. Further understanding of this method requires the systematic treatment of vertex corrections, an approach which is now vigorously pursued.

The LDA+ $\mathbf{U}$ is a method was introduced by Anisimov et al. ${ }^{62}$. It was made rotationally invariant in Refs. 63 and 64 . One can view this is as a special case of LDA+DMFT, where in the functional (Eq. (16)) $\Phi$ (the sum of graphs) is restricted to the Hartree-Fock graphs: $\Phi \rightarrow \Phi_{H F}$, and $\Sigma\left(\imath \omega_{n}\right)$ is replaced by a constant matrix $\lambda$. Then the LDA+U functional $\Gamma_{L D A+U}$ can be written in as follows:

$$
\begin{aligned}
& \Gamma_{L D A+U}\left[\rho(\vec{r}), n_{\alpha \beta}, V_{K S}(\vec{r}), \lambda_{\alpha \beta}\right]= \\
& -\operatorname{Tr} \ln \left(i \omega+\mu+\frac{\nabla^{2}}{2}-V_{K S}-\sum_{\alpha \beta \in L} \chi_{\alpha}^{*}(\vec{r}) \lambda_{\alpha \beta} \chi_{\beta}\left(\vec{r}^{\prime}\right)\right) \\
& -\int V_{K S}(\vec{r}) \rho(\vec{r}) d^{3} r-\lambda_{\alpha \beta} n_{\alpha \beta}+\int V_{c r y s t}(\vec{r}) \rho(\vec{r}) d^{3} r \\
& +E_{H}[\rho(\vec{r})]+E_{x c}^{L D A}[\rho(\vec{r})]+\Phi_{H F}\left[n_{\alpha \beta}\right]-\Phi_{D C}\left[n_{\alpha \beta}\right],
\end{aligned}
$$

where $n_{\alpha \beta}$ is the occupancy matrix. $\Phi_{H F}$ in Eq. (19) is the Hartree-Fock approximation

$$
\Phi_{H F}\left[n_{\alpha \beta}\right]=\frac{1}{2} \sum_{\alpha \beta \gamma \delta \in L}\left(U_{\alpha \gamma \delta \beta}-U_{\alpha \gamma \beta \delta}\right) n_{\alpha \beta} n_{\gamma \delta},
$$

where indexes $\alpha, \beta, \gamma, \delta$ refer to the fixed angular momentum $L$ of correlated orbitals, and the matrix $U_{\alpha \beta \gamma \delta}$ is the on-site Coulomb interaction matrix element.
Similarly to DMFT, the on-site Coulomb interaction is already considered within LDA approximately, so it is subtracted, hence the double-counting term $\Phi_{D C}$. One of the popular choices is so-called "fully-localized limit" (FLL) whose form is ${ }^{65}$

$\Phi_{D C}^{F L L}\left[n_{\alpha \beta}\right]=\frac{1}{2} \bar{U} \bar{n}(\bar{n}-1)-\frac{1}{2} \bar{J}\left[\bar{n}^{\uparrow}\left(\bar{n}^{\uparrow}-1\right)+\bar{n}^{\downarrow}\left(\bar{n}^{\downarrow}-1\right)\right]$,

where

$$
\begin{aligned}
\bar{n}^{\sigma} & =\sum_{\alpha \in L} n_{\alpha \alpha}^{\sigma}, \\
\bar{n} & =\bar{n}^{\uparrow}+\bar{n}^{\downarrow}, \\
\bar{U} & =\frac{1}{(2 L+1)^{2}} \sum_{\alpha \beta \in L} U_{\alpha \beta \beta \alpha}, \\
\bar{J} & =\bar{U}-\frac{1}{2 L(2 L+1)} \sum_{\alpha \beta \in L}\left(U_{\alpha \beta \beta \alpha}-U_{\alpha \beta \alpha \beta}\right) .
\end{aligned}
$$

The constant matrix $\lambda_{\alpha \beta}$ is determined by the saddle point equations $\frac{\delta \Gamma_{L D A+U}}{\delta n_{\alpha \beta}}=0$ :

$$
\begin{aligned}
\lambda_{\alpha \beta}= & \frac{\delta \Phi_{H F}}{\delta n_{\alpha \beta}}-\frac{\delta \Phi_{D C}}{\delta n_{\alpha \beta}} \\
= & \sum_{\gamma \delta}\left(U_{\alpha \gamma \delta \beta}-U_{\alpha \gamma \beta \delta}\right) n_{\gamma \delta} \\
& -\delta_{\alpha \beta}\left[\bar{U}\left(\bar{n}-\frac{1}{2}\right)-\bar{J}\left(\bar{n}^{\sigma}-\frac{1}{2}\right)\right] .
\end{aligned}
$$

The FLL double-counting term tends to work quite well for strongly correlated materials with very localized orbitals. However, for weakly correlated materials, FLL scheme describes the excessive stabilization of occupied states and leads to quite unphysical results such as the enhancement of the Stoner factor ${ }^{66}$. In order to resolve the problems, "around mean-field" (AMF) was introduced in Ref. 67 and further developed in Ref. 66 .

One can say that the LDA+U method works when correlations are static - and at the same time local. For example cases where magnetic or orbital order are very well developed. For a review of the $\mathrm{LDA}+\mathrm{U}$ method see Ref. 68.

Slave-Boson Method. The physics of strongly correlated electron materials requires to take into account on the same footing, localized - quasi-atomic degrees of freedom, which are important at high energies, together with strongly renormalized itinerant quasiparticles which emerge at low energy. DMFT captures this physics via a sophisticated quantum embedding that requires the solution of a full Anderson impurity model in a self consistent bath. A less accurate but computational faster methd to solve the strong correlation problem, which precedes DMFT is the Gutzwiller method which has been shown to be equivalent to the slave boson method in the saddle point approximation. This approach starts with an 
exact quantum many body problem, but one enlarges the Hilbert space so as to introduce explicitly operators which describe the different atomic multiplet configurations, and additional fermionic degrees of freedom which will be related to the emergent low energy quasiparticles. The method proceeded by writing a functional integral in the enlarged Hilbert space, supplemented by Lagrange multipliers which enforce multiple constraints. The approach, at zero temperature is very closely connected to the Gutzwiller method, which appears as a saddle point solution in the functional integral formalism ${ }^{69}$. In its original formulation this method was not manifestly rotationally invariant, but it was extended in this respect in Refs. 70-72. Further generalizations in the multi-orbital formulation and to capture non-local self-energies was introduced in Ref. 73, and we denote this formulation as the RISB (rotationally invariant slave boson) method. Within the slave particle method it is possible to go beyond mean field theory, and fluctuations around the saddle point generate the Hubbard bands in the one particle spectra $^{74}$. The RISB method can be used to compute the energy of lattice models. When in conjunction with the DMFT self-consistency condition, it gives the same results as the direct application of the method to the lattice model ${ }^{75}$. In this review, we will restrict ourselves to the RISB mean field theory, specifically from the perspective of the free-energy functionals that describe the free-energy of the system. We explain the physical meaning of the variables used in this method, and summarize succinctly the content of the mean field slave boson equations using a functional approach. A precise operational formulation of the method was only given recently ${ }^{76}$. For pedagogical reasons we start again with a Hubbard model with a tight binding one body Hamiltonian $H(\vec{k})$.

The variables used in RISB can be motivated by noticing that the many-body local density matrix $\rho$ (with matrix elements $\left.\left\langle\Gamma^{\prime}|\rho| \Gamma\right\rangle\right)$ admits a Schmidt decomposition, which can be written in terms of the expectation value of matrices of slave-boson operators $\phi_{B n}$ and $\phi_{B n}^{\dagger}$, which become $\phi_{B n}, \phi_{B n}^{*}$ when the single-particle index $\alpha$ is Mdimensional, and can be stored as $2^{M} \times 2^{M}$ matrices $\Phi$, $[\Phi]_{A n} \equiv \phi_{A n},\left[\Phi^{\dagger}\right]_{n A}=\phi_{A n}^{*}$, so that:

$$
\rho=\Phi \Phi^{\dagger}
$$

The method also introduces Fermionic operators $f_{\alpha}$ at each site (site indices are omitted in the following) which will represent the low energy quasiparticles at the mean field level. The physical electron operator $\underline{d}$ is represented in the enlarged Hilbert space by

$$
\underline{d}_{\alpha}=R_{\alpha \beta}[\phi] f_{\beta}
$$

where the matrix $\mathcal{R}$, with elements $R_{\alpha \beta}$ at the mean field level, has the interpretation of the quasiparticle residue, relating the physical electron to the quasiparticles. When $\mathcal{R}$ is small it exhibits the strong renormalizations induced by the electronic correlations. An important feature of the rotational invariant formalism is that the basis that diagonalizes the quasiparticles represented by the operators $f$ is not necessarily the same basis as that which would diagonalize the one electron density matrix expressed in terms of the operators $d$ and $d^{\dagger}$. Of central importance is the expression of the matrix $\mathcal{R}$, in terms of the bosonic amplitudes:

$$
\begin{aligned}
& R_{\alpha \beta}= \\
& \sum_{\gamma} \sum_{A B n m} F_{\alpha, A, B}^{\dagger} F_{\gamma, n m}^{\dagger}\left[\Phi^{\dagger}\right]_{n A}[\Phi]_{B m}\left[\left(\Delta^{p}\left(1-\Delta^{p}\right)\right)^{-1 / 2}\right]_{\gamma \beta} \\
& =\sum_{\gamma} \operatorname{Tr}\left[\Phi^{\dagger} F_{\alpha}^{\dagger} \Phi F_{\gamma}\right]\left[\left(\Delta^{p}\left(1-\Delta^{p}\right)\right)^{-1 / 2}\right]_{\gamma \beta} .
\end{aligned}
$$

We introduced here the matrices $F$,

$$
\left[F_{\alpha}\right]_{n m} \equiv_{f}\left\langle n\left|f_{\alpha}\right| m\right\rangle_{f}
$$

The matrices

$$
\Delta_{\alpha \beta}^{p} \equiv \sum_{A n m s}\left\langle m\left|f_{\alpha}^{\dagger}\right| s\right\rangle\left\langle s\left|f_{\beta}\right| n\right\rangle \Phi^{\dagger}{ }_{n A} \Phi_{A m}=\operatorname{Tr}\left[F_{\alpha}^{\dagger} F_{\beta} \Phi^{\dagger} \Phi\right]
$$

have the physical interpretation of a quasiparticle density matrix:

$$
<f_{\alpha}^{\dagger} f_{\beta}>=\Delta_{\alpha, \beta}^{p}
$$

For a multi-band Hubbard model with a tight-binding one-body Hamiltonian $H(\vec{k})$ and interactions $\Sigma_{i} H_{i}^{l o c}$, the RISB functional, whose extremization gives the slaveboson mean field equations, is expressed in terms of $\phi_{i}, \phi_{i}^{\dagger}$ (the slave-boson amplitude matrices) and the matrices $\lambda_{i}^{c}, \lambda_{i}, \mathcal{D}$. These are $N \times N$ matrices of Lagrange multipliers: (i) $\lambda_{i}^{c}$ enforces the definition of $\Delta_{i}^{p}$ in terms of the RISB amplitudes (ii) $\lambda_{i}$ enforces the Gutzwiller constraints and (iii) $\mathcal{D}_{i}$ enforces the definition of $\mathcal{R}_{i}$ in terms of slave boson amplitudes. Another variable, $E^{c}$ enforces the normalization $\operatorname{Tr}\left[\Phi \Phi^{\dagger}\right]=1$.

The variables $\mathcal{R}, \lambda$ can be thought as a parametrization of the self-energy. While the matrices $\mathcal{D}, \lambda^{c}$ are a parametrization of a small impurity model (the dimension of the bath Hilbert space is the same as that of the impurity Hilbert space), $\mathcal{D}$ is the hybridization function of the associated impurity model while $\lambda^{c}$ parametrizes the energy of the bath. $\Delta^{p}$ describes the quasiparticle occupancies, which are the static analogs of the impurity quasiparticle Green's function.

The RISB (Gutzwiller) functional for a model Hamiltonian with a local part which is bundled together with a local interaction term in $H^{l o c}$ and a kinetic energy matrix which is non-local $H(\vec{k})^{\text {nonloc }}$, was constructed in Ref. 75: 


$$
\begin{aligned}
& \Gamma_{\text {model }}\left[\phi, E^{c} ; \mathcal{R}, \mathcal{R}^{\dagger}, \lambda ; \mathcal{D}, \mathcal{D}^{\dagger}, \lambda^{c} ; \Delta^{p}\right]= \\
& -\lim _{\mathcal{T} \rightarrow 0} \frac{\mathcal{T}}{\mathcal{N}} \sum_{k} \sum_{m \in \mathbb{Z}} \operatorname{Tr} \ln \left(\frac{1}{i(2 m+1) \pi \mathcal{T}-\mathcal{R} H(\vec{k})^{\text {nonloc }} \mathcal{R}^{\dagger}-\lambda+\mu}\right) e^{i(2 m+1) \pi \mathcal{T} 0^{+}} \\
& +\sum_{i} \operatorname{Tr}\left[\phi_{i} \phi_{i}^{\dagger} H_{i}^{l o c}+\sum_{a \alpha}\left(\left[\mathcal{D}_{i}\right]_{a \alpha} \phi_{i}^{\dagger} F_{i \alpha}^{\dagger} \phi_{i} F_{i a}+\text { H.c. }\right)+\sum_{a b}\left[\lambda_{i}^{c}\right]_{a b} \phi_{i}^{\dagger} \phi_{i} F_{i a}^{\dagger} F_{i b}\right]+\sum_{i} E_{i}^{c}\left(1-\operatorname{Tr}\left[\phi_{i}^{\dagger} \phi_{i}\right]\right) \\
& -\sum_{i}\left[\sum_{a b}\left(\left[\lambda_{i}\right]_{a b}+\left[\lambda_{i}^{c}\right]_{a b}\right)\left[\Delta_{i}^{p}\right]_{a b}+\sum_{c a \alpha}\left(\left[\mathcal{D}_{i}\right]_{a \alpha}\left[\mathcal{R}_{i}\right]_{c \alpha}\left[\Delta_{i}^{p}\left(1-\Delta_{i}^{p}\right)\right]_{c \alpha}^{\frac{1}{2}}+\text { c.c. }\right)\right] .
\end{aligned}
$$

This method can also be turned into an ab-initio $\mathrm{DFT}+\mathrm{G}$ method (or DFT+RISB). To motivate the construction of a DFT+G functional we simply follow the same path used above to go from the model DMFT Hamiltonian to a DFT+DMFT functional. We substitute $H(\vec{k})$ for $-\frac{1}{2} \nabla^{2}+V_{K S}(\vec{r})$, which has a local and a nonlocal part, and follow the same steps as in the DFT+DMFT section.

$$
\begin{aligned}
& \Gamma_{D F T+G}\left[\rho(\vec{r}), V_{K S}(\vec{r}), \phi, E^{c} ; \mathcal{R}, \mathcal{R}^{\dagger}, \lambda ; \mathcal{D}, \mathcal{D}^{\dagger}, \lambda^{c} ; \Delta_{i}^{p}\right]= \\
& \Gamma_{\text {model }}\left[H(\vec{k}) \rightarrow-\frac{1}{2} \nabla^{2}+V_{K S}(\vec{r})\right]+\Gamma_{2}\left[V_{K S}(\vec{r}), \rho(\vec{r})\right]- \\
& \quad \sum_{i} \Phi_{D C}\left[\Delta_{i}^{p}\right]
\end{aligned}
$$

where $\Gamma_{2}$ and $\Phi_{D C}$ are the same functionals defined in the subsection on DMFT. The LDA+RISB and the $\mathrm{LDA}+\mathrm{G}$ method are completely equivalent (more precisely, the slave boson method has a gauge symmetry, and a specific gauge needs to be chosen to correspond to the multi-orbital Gutzwiller method introduced in Ref. 77. DFT $+\mathrm{G}$ was formulated in Refs. 78 and 79. The slave boson method in combination with DFT was first used in Ref. 80 in a non-rotationally-invariant framework and with full rotational invariance in Ref. 73. For a recent review see Ref. 81.

Comparing the methods, critical discussion, future directions and outlook

For weakly correlated systems we argued in section II that once the structure is known, we have a well-defined path to compute their properties using DFT and the $G_{0} W_{0}$ method. To go beyond requires to move in the space illustrated in Fig 3. This has to be done while respecting as many general properties such as conservation laws (Refs.82-84), sum rules, unitarity and causality (Refs.85-87) as possible. This is a very difficult problem which is under intensive investigation.

This section reviewed several Green's-function-based approaches available for studying strongly-correlatedelectron materials. The reader may wonder why we considered multiple methods. There are two reasons. First, as stressed throughout the paper and demonstrated in the examples, presented in the next sections there are materials where correlations are mostly static, and others where dynamical correlations dominate the physics. These different types of correlations require different methods. Second, even when two methods treat the same type of correlations, they have different accuracies and computational speeds. Finding the correct trade-off between speed and accuracy will be important, in particular when high throughput studies start becoming feasible for strongly correlated systems.

As we strive towards a fully controlled but practical solution of the full many-body problem for solid state physics, we will need more exact and thus slower methods to benchmark the faster but more practical ones. Hence it is important to compare them and understand their connection. Static correlations can be treated by GW methods, and one can view the hybrid-functional exchange-correlation potentials as faster approximations to the QPGW exchange / correlation potential. One can also assess whether the GGA (or LDA) exchange / correlation potential is a good approximation to the selfenergy in a given material - by checking how close it is to the corresponding self-consistent QPGW exchange correlation potential.

In the same spirit one can understand the successes of LDA+DMFT from the $\mathbf{G W}+\mathbf{D M F T}$ perspective. One issue is the definition of $U$ in a solid. The functional $\Phi$ can be viewed as the functional of an Anderson impurity model which contains a frequency-dependent interaction $U(\omega)$ obeying the self-consistency condition:

$$
U^{-1}=W_{l o c}^{-1}+\Pi_{l o c} .
$$

This provides a link between LDA+DMFT, which uses a parameter $U$, and the $\mathrm{GW}+\mathrm{DMFT}$ method, where this quantity is self-consistently determined. An important question is thus under which circumstances one can approximate the Hubbard $U$ by its static value. For projectors constructed on a very broad window, $U(\omega)$ is constant on a broad range of frequencies ${ }^{88}$. An important open question is how one can incorporate efficiently the effects of the residual frequency dependence of this interaction.

Another question is the validity of the local ansatz for graphs beyond the $G W$ approximation. This question was first addressed in Ref. 89, who showed that the lowest order $G W$ graph is highly non-local in all semicon- 
ductors, which can be understood as the exchange Fock graph is very non-local. On the other hand, higher-order graphs in transition metals in an LMTO basis set were shown to be essentially local.

Consider a system such as Cerium, containing light spd electrons and heavier, more correlated, $f$ electrons. We know that for very extended systems, the GW quasiparticle band structure is a good approximation to the LDA band structure. Therefore the self-energy of a diagrammatic treatment of the light electrons can be approximated by the exchange-correlation potential of the LDA (or by other improved static approximations if more admixture of exchange is needed) . Diagrams of all orders but in a local approximation are used for the $f$ electrons. In the full many-body treatment $\Sigma_{f f}$ is computed using skeleton graphs with $G_{l o c}$ and $W_{l o c}$. To reach the LDA+DMFT equations, one envisions that at low energies the effects of the frequency dependent interaction $U(\omega)$ can be taken into account by a static $U$, which should be close to (but slightly larger than) $U(\omega=0)$. The $f f$ block of the Green's function now approaches $\Sigma_{f f}-E_{d c}$.

We reach the LDA+DMFT equations with some additional understanding on the origin of the approximations used to derive them from the GW+DMFT approximation, as summarized schematically in

$$
\begin{aligned}
& \Sigma_{G W+D M F T}(\vec{k}, \omega) \longrightarrow \\
& \left(\begin{array}{cc}
0 & 0 \\
0 & \Sigma_{f f}-E_{d c}
\end{array}\right)+\left(\begin{array}{cc}
V_{x c}[\vec{k}]_{s p d, s p d} & V_{x c}[\vec{k}]_{s p d, f} \\
V_{x c}[\vec{k}]_{f, s p d} & V_{x c}[\vec{k}]_{f, f}
\end{array}\right) .
\end{aligned}
$$

Realistic implementations of combinations of GW and DMFT have not yet reached the maturity of LDA+DMFT implementations, and are a subject of current research. Recent self-consistent implementations include Refs. 60 and 90.

When strong dynamical correlations are involved, the spectra is very far from that of free fermions. The one electron spectral function $A(\vec{k}, \omega)$ displays not only a dispersive quasiparticle peak, but also other features commonly denoted as satellites. The collective excitation spectra, which appear in the spin and charge excitation spectra, does not resemble the particle-hole continuum of the free Fermi gas with additional collective (zero sound, spin waves) modes, produced by the residual interactions among them. Finally the damping of the elementary excitations in many regimes does not resemble that of a Fermi liquid. Strong dynamical correlations are accompanied by anomalous transport properties, large transfer of optical spectral weight, large mass renormalizations, as well as metal-insulator transitions as a function of temperature or pressure. These can be captured by DMFT, which combined with electronic structure, enable the treatment of these effects in a material-specific setting, but not by $\mathrm{LDA}+\mathrm{G}$ which only provides a quasiparticle description of the spectra. Many successful comparisons with experimental ARPES and optical and neutron scattering data have been made over the last two decades using LDA+DMFT which makes an excellent compromise of accuracy for speed, and it is now the mainstay for the elucidation of structure property relations in strongly correlated materials. $\mathrm{LDA}+\mathrm{G}$ can only describe at best the quasiparticle featurs in that spectra.

On the other hand, as it will be stressed through examples, for total energy evaluations - which are a central part of the material design workflow, faster methods are currently needed. We described above two methods, the LDA+U method, and the Gutzwiller RISB method, which fall in this "fast but less accurate" category. These methods can be viewed as approximations to the many body problem within a DMFT perspective. As pointed out in Refs. 75 and 91, the Gutzwiller RISB leads to a DMFT-like impurity solver with a bath consisting of only one site. LDA $+\mathrm{U}$ can be viewed as a limiting case of DMFT, where a static local self-energy is considered.

There are numerous algorithmic challenges in optimizing studies of materials based on DMFT. While CTQMC runs for solving the Anderson impurity model, i.e. the single orbital case, as well as 3 or 2 orbitals $\left(t_{2 g}\right.$ and $e_{g}$ electrons) can be completed on one CPU in less than one day for extremely low temperatures, a full d-shell (5 electrons) requires several days, and the full f-shell is still at the border of what can be done with current methods. All this assumes high symmetry situations, where the hybridization function is diagonal. Off-diagonal hybridization introduces severe minus sign problems. Alternative exact diagonalization-based methods, such as NRG or DMRG will be needed. This would also help with the problem of reducing the uncertainties involved in the process of analytic continuation.

While the ansatz 10 has reproduced the photoemission spectra of many materials, there have not been highthroughput studies which would enable us to systematically search for deviations. This requires the improvement of computational tools, an area of active research. What if the $\boldsymbol{k}$ and $\omega$ dependencies cannot be disentangled? This situation may arise near a quantum critical point. Methods to incorporate the non-local correlations beyond DMFT are an important subject of active research, which is reviewed in Ref. 59.

Armed with an understanding of methods to treat correlations and their physical and computational trade-offs, we proceed in section IV to construct a workflow for designing correlated materials.

\section{MATERIALS DESIGN WORKFLOW}

Condensed matter physics has a long standing tradition of constant interplay between theory and experimentation. The field of strongly correlated electron systems, has been driven by unexpected experimental discovery a new materials, followed by a large number of theoretical ideas which get refined as new experimental information becomes available. This is described in Fig. 9 panel 
(a)

(b)

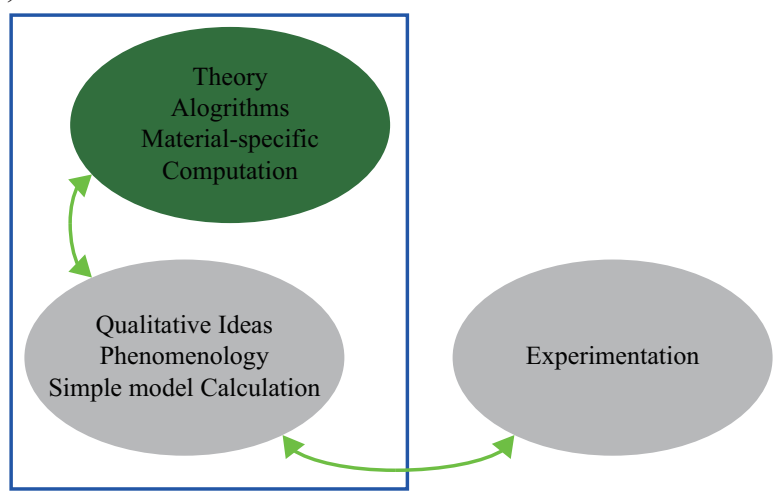

FIG. 9. (a) Traditional theory experiment theory interaction. This loop is initiated by an experimental discovery for which many theories are proposed and corrected by further experimentation. (b) The modern material design loop augments the theoretical contribution, now this loop can be initiated by either theory or experiment.

(a). Panel (b) describes how theory, algorithms and computational power have enhanced theoretical capabilities, which make the approach material-specific, thus enabling theory-assisted material design.

At the current state of development, the material design process for strongly correlated electron compounds should begin at the intuitive level, for example some physical idea of model that one would like to explore or test, a property of a strongly correlated material which could result in a useful property, a class of compounds one would like to investigate comparatively, some ideas of chemistries of strongly correlated materials which would enhance desirable solid state properties. This zeroth order step can be refined with simplified quantitative calculations using model Hamiltonians, or other computational tools which refines our intuition. After this zeroth order step, it is natural to divide the process of materials design into three additional steps as summarized in Table II, which will be illustrated by example in the following sections of this paper.

The first step is the quantitative calculation of the electronic structure, namely, how to go from a well defined structure (i.e. atomic positions and ionic charges) to physical or chemical properties. Given a crystal structure, we seek to compute one or several electronic properties such as orbital occupancies, transport coefficients (resistivities, mobilities, thermoelectric coefficients, etc.) Mott or charge transfer gap sizes, magnetic order parameters, etc. As we have seen in section II, for correlated electron materials the computational method used for electronic structure depends on the strength and kind of correlations and should be chosen appropriately. Sometimes, this step is divided into two. In the first step first one derives from first principles an effective model Hamiltonian, and then one solves the model and explores its consequences. While the advanced functionals described in the previous section go directly from structure to property bypassing the model Hamiltonian, the latter can be useful for the interpretation of the results.

The second step is structure prediction: predict the crystal structure given a fixed chemical composition. A successful prediction would take a formula, like $\mathrm{Fe}_{2} \mathrm{O}_{3}$ for example, and return the correct crystal structure-in this case, the composition forms in the corundum structure, called the $\alpha$ phase. For a more complete characterization, we would seek to predict not only the ground state structure, but nearby local minima as well, termed polymorphs. Again taking $\mathrm{Fe}_{2} \mathrm{O}_{3}$ as an example, this composition also forms in the spinel structure, found naturally as the mineral maghemite and termed the $\gamma$ phase, as well as cubic $\beta$ and orthorhombic $\epsilon$ phases. Polymorphs generally are formed in different temperature and pressure regimes, and modeling these effects add an additional layer of complexity. However, simply enumerating the low-energy local minima at zero temperature can already provide a broader picture of the structural diversity of a composition. Furthermore, if the structure one is interested turns out not to be the ground state but a metastable structure one can design methods for stabilizing it, either by choosing an appropriate synthesis method or by applying external perturbations such as stress exerted by a properly chosen substrate.

The general procedure for structure prediction involves placing atoms in a unit cell and using an algorithm to efficiently traverse the space of atomic configurations and cell geometries to arrive at low energy structures. This step requires having an accurate method for producing the energy of a given configuration of atoms and sampling these configurations. There are a number of structure prediction techniques (see the review Ref. 92) including the simulated annealing approach ${ }^{93,94}$, evolutionary algorithm methods ${ }^{95-97}$, structure models by analogy based on data mining and machine learning 98,99 , metadynamics ${ }^{100,101}$, basin and minima hopping ${ }^{102,103}$, random structure searching ${ }^{104,105}$, and so on.

The third step is testing for global stability: given the lowest energy structure of a fixed composition, check whether it is stable against decomposition to all other compositions (phase separation) in the chemical system.

The steps involving total energies are assisted by electronic structure methods and material databases. In particular the third step which requires the knowledge of all other known stable compositions, their crystal structures and total energies, is now facilitated by materials databases containing data in standardized computable formats, such as the Materials Project ${ }^{3}$, the Open Quantum Materials Database (OQMD) ${ }^{4}$, AFLOWlib $^{5}$ and NIMS $^{6}$. With this information, the energetic convex hull for a chemical system can be constructed and the target 


\begin{tabular}{l|c|c}
\hline Name & Step & Tools \\
\hline Motivation and analysis & $\begin{array}{c}\text { defining directions and hypotheses to be tested, } \\
\text { refining them with simplified computations }\end{array}$ & Heuristics, theory, simplified computations \\
\hline \hline $\begin{array}{l}\text { electronic structure } \\
\text { structure prediction } \\
\text { global stability }\end{array}$ & $\begin{array}{c}\text { structure } \rightarrow \text { property } \\
\text { composition } \rightarrow \text { structure } \\
\text { chemical system } \rightarrow \text { composition }\end{array}$ & $\begin{array}{c}\text { electronic structure codes, DFT, DFT+DMFT } \\
\text { evolutionary algorithms, Monte Carlo, minima hopping } \\
\text { convex hull from materials databases }\end{array}$ \\
\hline
\end{tabular}

TABLE II. Three step workflow of materials design. Electronic structure and structure prediction have been accessible for decades, with density functional theory (DFT) as the key underlying method. In contrast, checks for global stability require knowledge of all other structures within a chemical system, which was only possible after the creation of extensive computational materials databases.

composition checked for stability against decomposition (phase separation).

For weakly-correlated materials, the entire workflow can be built around $L D A / G G A$ for total energies and $G_{0} W_{0}$ for spectral properties. For correlated materials, GGA is a good starting point for computing total energy differences (such as reaction energies or structural energy differences). In this review we highlight some failures of the LDA/GGA energies in structural prediction and phase stability, and ways to introduce corrections to account for the correlation effects.

The need to correct DFT total energies for materials design projects, is broadly recognized in the context of all the material databases where the GGA/LDA results are corrected using semi-empirical schemes. There are three broadly used schemes in literature, and they have associated databases, the fitted elemental-phase reference energy (FERE) scheme ${ }^{14}$, Materials Project ${ }^{3}$, and Open Quantum Materials Database (OQMD) ${ }^{4}$.

While the details of the implementation are different, they have two key elements in common. First, instead of using GGA, the total energies are computed within the GGA+U method, with some $\mathrm{U}$ values empirically assigned to each element. Second, the experimental formation energies $\Delta H^{\exp }$ are used to determine best fits for elemental energies $E^{\text {Fitted }}(A)$, where $\mathrm{A}$ is an element, for a training set of compounds by solving the linear leastsquares problem.

$$
\begin{aligned}
& \Delta H^{\exp }\left(\mathrm{A}_{m} \mathrm{~B}_{n}\right) \approx \mathcal{E}_{\text {corrected }}^{\mathrm{GGA}+\mathrm{U}}\left(\mathrm{A}_{m} \mathrm{~B}_{n}\right) \\
& =E^{\mathrm{GGA}+\mathrm{U}}\left(\mathrm{A}_{m} \mathrm{~B}_{n}\right)-m E^{\text {Fitted }}(A)-n E^{\text {Fitted }}(B)
\end{aligned}
$$

We note that all elemental energies are fitted for all elements in FERE (whithin the set of relevant elements) while only selected ones are fitted in MP and OQMD (especially in the "fit-partial" scheme in OQMD).

We describe the different correction methods in Appendix A. In this article we will use the Materials Project database for analysis of phase stability and estimate probabilities based on their data. We will show in section VIE that careful consideration of correlations is essential not only for calculation of phase stability, but also for structure prediction.

Notice that the theoretical workflow, outlined in Table II progresses in an order different from experimen- tal solid state synthesis. There, elements and simple compounds in a chemical system are combined and subjected to heating/cooling cycles to provide the kinetic energy necessary for atomic rearrangement to form new stoichiometries (of which there may be more than one). Finally the stoichiometries crystallize to form structures which are then isolated for the study of their properties.

\section{STATISTICAL INTERPRETATION}

DFT has reached a high degree of stability and scalability, enabling software packages such as USPEX ${ }^{96,106}$ to implement genetic algorithms on top of DFT to successfully predict never before observed structures. As discussed in the previous sections, correlations in the form of $\mathrm{U}$ and empirical corrections are now available in several databases. Since these methods are not exact methods and suffer from systematic errors, compounds predicted to be stable will not necessarily be found in experiment, and vice versa.

The main question we address in this section is the interpretation of the above-hull/below-hull energies that we compute within LDA/GGA (with or without the empirical corrections). There are two related questions: (1) what is the likely error in the computed energy, (2) how likely is the compound to be synthesized given its energy relative to the convex hull. Namely, how likely is one to find the target compound? This assessment serves as a background for the conclusion section VII, where we evaluate the results of various material design projects.

Reference 107 modeled the computational error - the difference between computed and experimental formation energies - as a random variable with normal distribution. A normal-distribution was also used by Ref. 4, as seen in Fig. 11. We follow a similar statistical approach to the question.

Denote by $\mathcal{E}_{\text {exp }}$ the experimental heat or enthalpy per atom of the reaction $\mathrm{A}+\mathrm{B} \rightarrow \mathrm{AB}$ at low temperature, and denote by $\mathcal{E}_{\text {calc }}$ the same quantity computed using an approximate method, like GGA, or the empiricallycorrected value of this computation. We treat $\mathcal{E}_{\text {calc }}$ and $\mathcal{E}_{\text {exp }}$ as real random variables, and analyze the distribution of the variable $d=\mathcal{E}_{\text {calc }}-\mathcal{E}_{\text {exp }}$ : 


$$
P\left(\mathcal{E}_{\text {calc }}-\mathcal{E}_{\text {exp }}=d\right)=F_{\alpha \beta \mu}(d)
$$

where $F_{\alpha \beta \mu}(d)$ is some probability distribution function (PDF) with center $\mu$ and scale $\alpha$, as well as some shape parameter $\beta$. Since GGA (or one of its correction schemes) is reasonably accurate, we expect $F_{\alpha \beta \mu}(d)$ to be concentrated around the center.

In order to study this distribution, we observe that the same quantity in Eq. (28) describes computational errors in energies above-the-hull as well as computational errors in formation energies. This holds because the distribution applies to computational error in reaction energies in general. The crucial point that makes this possible is that the number of atoms is balanced on the left and on the right (so as to cancel core energies). Therefore we can train a statistical model on the distribution of computational error for formation energies, for which there exists a reasonably-sized experimental data set, and then make predictions based on above-hull energies.

We expect a stronger statistical-correlation when all the systems $\mathrm{A}, \mathrm{B}$, and $\mathrm{AB}$ are weakly correlated than when the correlations are strong, hence the parameters $\alpha, \beta, \mu$ should be taken in a well-defined space of materials defined from the outset. Since we will use this model for prediction, it is necessary to fit the parameters using a large-enough sample of representative materials.

Figure 10 shows the distribution of computational error $\mathcal{E}_{\text {calc }}-\mathcal{E}_{\text {exp }}$ for formation energies of compounds collected in the data set. The data set includes 1,500 substances from the OQMD database and the materials listed in table II in [14]. It is noteworthy that the experimental formation energies are available as well as crystal structures of the compounds in the OQMD database (query ${ }^{108}$ was used to collect these pairs). The experimental data originates from 2 sources: the SGTE Solid SUBstance (SSUB) database ${ }^{109}$, and the thermodynamic database at the Thermal Processing Technology Center at the Illinois Institute of Technology (IIT) ${ }^{110}$, as described in Ref. 4. The experimental formation energies were also collected from table II in 14 and were merged with the data set. In Fig. 10 the left-side plots show the computational error for pure GGA calculations (reproduced with our own VASP calculations for the compounds in the data set), whereas the right hand side includes $+\mathrm{U}$ for some of the compounds, as in the from the Materials Project's recipe (see appendix for details). We observe that the distribution is skewed to the right, and that application of the $\mathrm{U}$ correction is not enough to undo the skewness, although it reduces $\sigma$ from $427 \mathrm{meV}$ to $266 \mathrm{meV}$ (with an increase of the mean error from 155 $\mathrm{meV}$ to $172 \mathrm{meV}$ ). This data is summarized in Table III.

As discussed above, various authors have corrected the GGA/GGA+U values for formation energies by shifting the chemical potentials of elements. This is usually achieved by fitting the set of experimental results on the equations for GGA/GGA + U formation energies as in Eq. (27). The effect of this procedure on the distribution
$F_{\alpha \beta \mu}(d)$ is to make it centered, and to eliminate the drift of the bivariate distribution. This can be seen in the OQMD fit (Fig. 11, as well as in FERE and Materials Project (Fig.15).

Another important property of the corrected distributions is that the error can be seen as approximately independent of the value of the computed energy:

$$
\begin{gathered}
P\left(\mathcal{E}_{\text {calc }}-\mathcal{E}_{\text {exp }}=d \mid \mathcal{E}_{\text {calc }}=x\right) \approx P\left(\mathcal{E}_{\text {calc }}-\mathcal{E}_{\text {exp }}=d\right) \\
=F_{\alpha \beta \mu}(d)
\end{gathered}
$$

This (approximate) independence is evident in Fig. 12. One can reason that as long as the computational error is small, it should not be correlated with the value of the computed energy. Finally, with just a few 1000's of points, there is not enough data to split the domain into sub-ranges and make meaningful statistical analysis. With more data one could refine the distribution parameters on ranges of $\mathcal{E}_{\text {calc }}=x$, or possibly other variables that we did not consider here.

For prediction, the probability that $A B$ forms as the ground state, when the computed energy is at a distance $x$ above the Hull is given by

$$
\begin{aligned}
\mathcal{P}(x) & =\int_{-\infty}^{0} P\left(\mathcal{E}_{\text {exp }}=y \mid \mathcal{E}_{\text {calc }}=x\right) d y \\
& =\int_{-\infty}^{0} P\left(\mathcal{E}_{\text {calc }}-\mathcal{E}_{\text {exp }}=x-y \mid \mathcal{E}_{\text {calc }}=x\right) d y \\
& \approx \int_{-\infty}^{0} F_{\alpha \beta \mu}(x-y) d y=1-\mathcal{F}_{\alpha \beta \mu}(d \leqslant x)
\end{aligned}
$$

where $\mathcal{F}_{\alpha \beta \mu}(\mathrm{d} \leqslant \mathrm{x})$ is the cumulative distribution function corresponding to $F_{\alpha \beta \mu}$. This expression can be evaluated numerically by estimating the number of data points in the distribution tail (where \# denotes the number of elements in the set):

$$
\mathcal{P}(x) \approx \frac{\#\left\{\left(\mathcal{E}_{\text {calc }}, \mathcal{E}_{\text {exp }}\right) \mid \boldsymbol{E}_{\text {calc }}>x\right\}}{\#\left\{\left(\mathcal{E}_{\text {calc }}, \mathcal{E}_{\text {exp }}\right)\right\}},
$$

however, it is convenient to have an analytic form. For the corrected distributions, we postulate that $F_{\alpha \beta \mu}$ is Normal or generalized-Normal distribution - which includes also Normal $(\beta=2)$, exponential $(\beta=1)$, as well as uniform $(\beta=\infty)$ distributions:

$$
F_{\alpha \beta \mu}(d)=\frac{\beta}{2 \alpha \Gamma(1 / \beta)} e^{-(|d-\mu| / \alpha)^{\beta}}
$$

Using the experimental data (see above), we calculated the numbers listed in Table III. We used the maximum likelihood method to estimate $\alpha, \beta$ and $\mu$. The first column summarizes the distribution of raw GGA calculations, which were reproduced with our own GGA runs for the compounds in the data set. The second column corresponds to Materials Project's GGA or GGA+U (GGA 

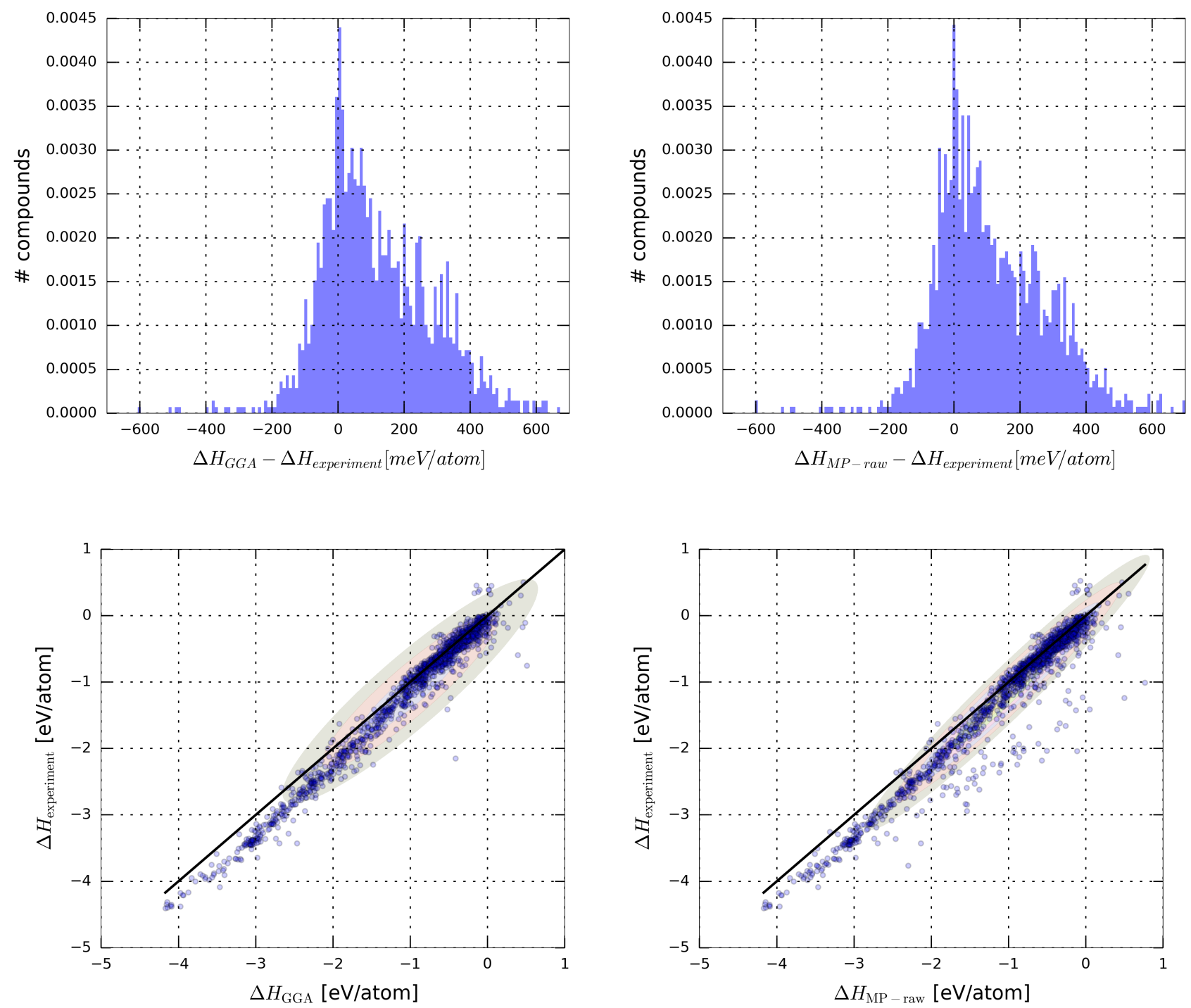

FIG. 10. Left: Distribution of computational error reproduced with VASP GGA(PBE) for compounds collected in the data set. The data set includes 1,500 substances from OQMD and the materials listed in table II in 14. To get the experimental formation energies for the corresponding compounds in the data set, we use the OQMD database, where the experimental data is available as well as the crystal structure. The experimental data is also merged with table II in 14. The pure GGA(PBE) formation energies are calculated for the compounds in the data set and compared with the corresponding experimental formation energies. Right: plots for Materials Project's raw data, which adds nonzero U for some elements in certain compounds. As can be seen, addition $\mathrm{U}$ is not enough to make the distribution $F_{\alpha \beta \mu}(d)$ un-skewed or fix the drift to the bottom (this is also evident in the Fit-none distribution from $\mathrm{OQMD}^{4}$, shown in Fig. 11). Also shown are the ellipses of the bivariate-normal estimators, demonstrating the drift.

for most compounds and GGA+U only for certain correlated materials specified in appendix), which was evaluated using the raw data from Materials Project. These distributions are depicted in Fig. 10. We did not include model estimates for the non-corrected distributions, since they do not comply with some of the assumptions (as explained above). The third column in the table is fitted for $\triangle H_{F E R E}$ from Table II in Ref. 14. As expected, the parameters for FERE show a smaller standard error (for a smaller dataset). The fourth column corre- sponds to corrected formation-energies from the Materials Project. Again, the parameters show a smaller standard error compared to bare GGA. These distributions are depicted in Fig. 15. Figure 14 shows the calculated probabilities in each one of the schemes for $\mathcal{E}_{\text {calc }}$.

Interestingly, the mean values for pure GGA and Materials Project's raw data (table III) are positive, meaning that GGA/GGA+U tend to over-estimate formation energies. We also observe that the corrected distributions are more exponential-like then normal. In fact, they all 

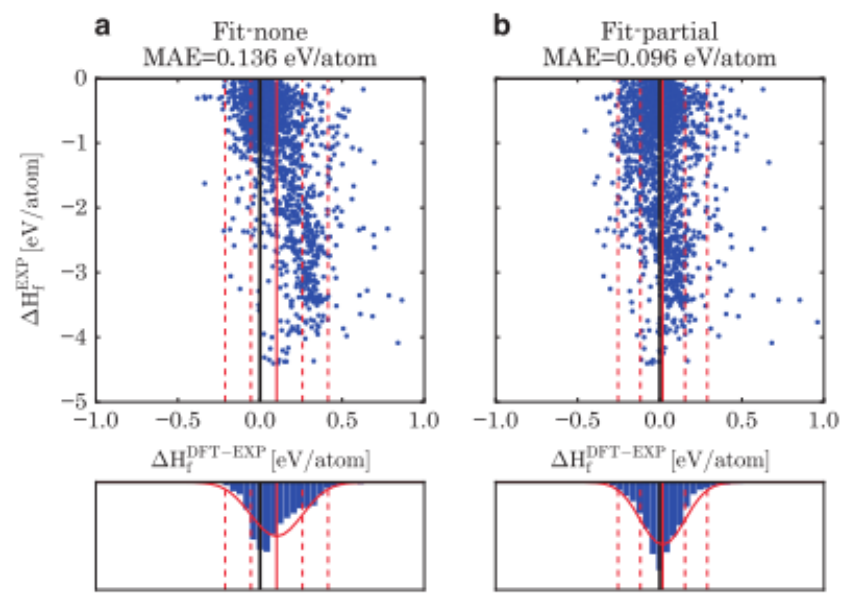

FIG. 11. Comparison of "Fit-None" to "Fit-Partial" in OQMD (from Ref. 4). Left side shows raw data (with U added to some correlated elements in certain compounds) there is an evident skewness and drift. On the right the data is corrected by a small set of chemical potentials. The skewness and drift are eliminated.

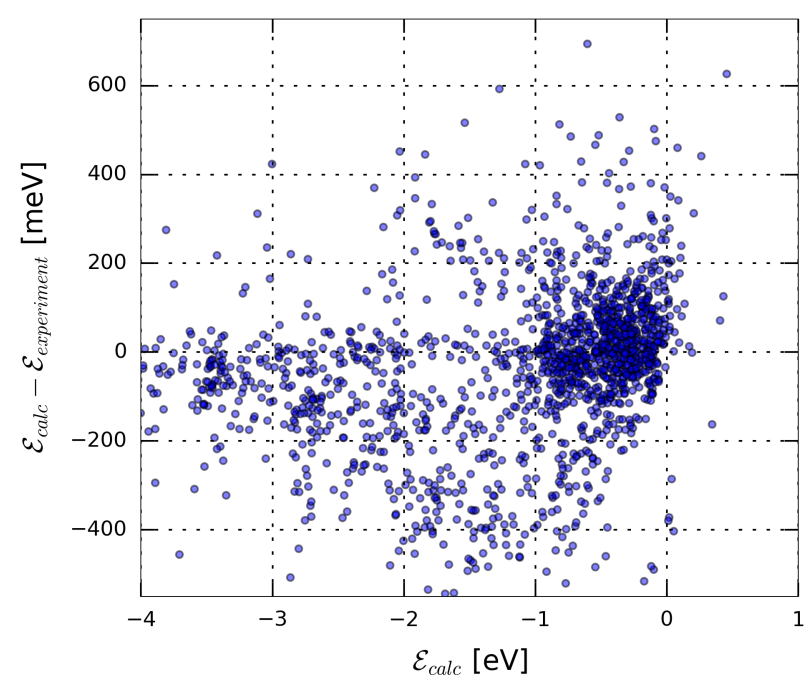

FIG. 12. Plot of the computational error in Materials Project formation energies against the calculated formation energy, demonstrating little or no correlation between $\mathcal{E}_{\text {exp }}-\mathcal{E}_{\text {calc }}$ and $\mathcal{E}_{\text {calc }}$. Each data point corresponds to a pair of calculated and experimental formation energy for a material in the data set.

get a very low score on statistical Shapiro testing for Normalcy, but pass the Kolmogorov test for generalizedNormal distribution.

In general we would like to apply the same kind of statistical error analysis to the systematic computational error made by correlated electronic structure calculations, such as DMFT, or Gutzwiller. However, since there exists no database of energies for these methods, at this point in time it is impossible to estimate the parameters of the model Eq. (30) for correlated methods. Still,

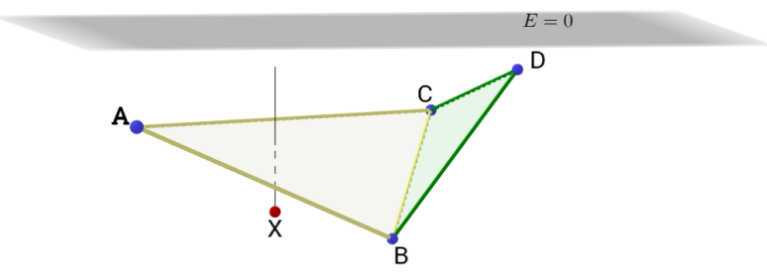

FIG. 13. The determinant reaction for a new material $\mathrm{X}$ - X lies either above or below the convex hull of reactions. In the illustration above it is well below the $\mathrm{ABC}$ triangle, although its energy is above the $\mathrm{B}$ compound (note that in this case $\mathrm{X}$ still forms out of $\mathrm{A}, \mathrm{B}$, and $\mathrm{C}$ ). X's computed distance below the hull, which we denoted in the text by $\mathcal{E}_{X}$, is the quantity that determines its formation probability in our analysis. In this diagram it is equal to X's vertical distance from the triangle $\mathrm{ABC}$, and therefore the reaction $\mathrm{ABC} \rightarrow \mathrm{X}$ is the determinant reaction in this hull.

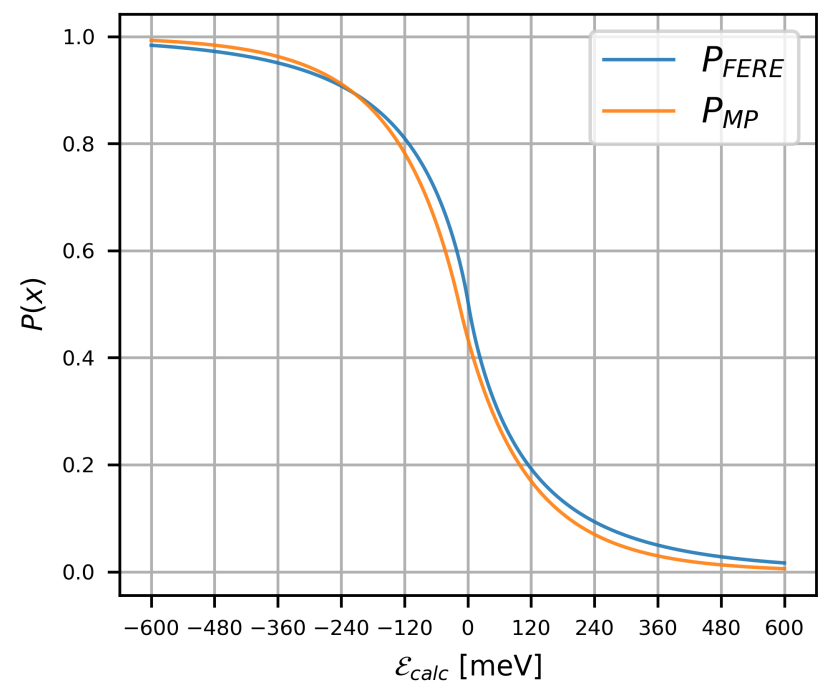

FIG. 14. $\mathcal{P}\left(\mathcal{E}_{\text {calc }}\right)$, probability for a compound to exist, given the computed formation energy $\mathcal{E}_{\text {calc }}$ with the two correction schemes summarized in Table III.

since we expect these methods to be more accurate than GGA, we expect their standard error $\sigma$ to be smaller, and their mean $\mu$ to be closer to 0 than what we found for GGA. Since our probability estimate depends only on the distance from the convex hull, it is determined only by one triangle in the diagram, which we refer to as the determinant reaction, see Fig. 13. Only the energies of the relevant compounds (in the diagram, these are $\mathrm{A}, \mathrm{B}, \mathrm{C}$, and $\mathrm{X}$ ) need to be re-estimated using the computationally expensive correlated method, and thus one can improve the probability estimate for X, without requiring too much extra computation. Since the cumulative distribution function (Eq. (29)) for the correlated computation will be sharper, if the energy of $\mathrm{X}$ falls below the hull, its probability will clearly be larger than 0.5 , whereas if the energy is well above the hull we argue that it is unlikely to exist. 

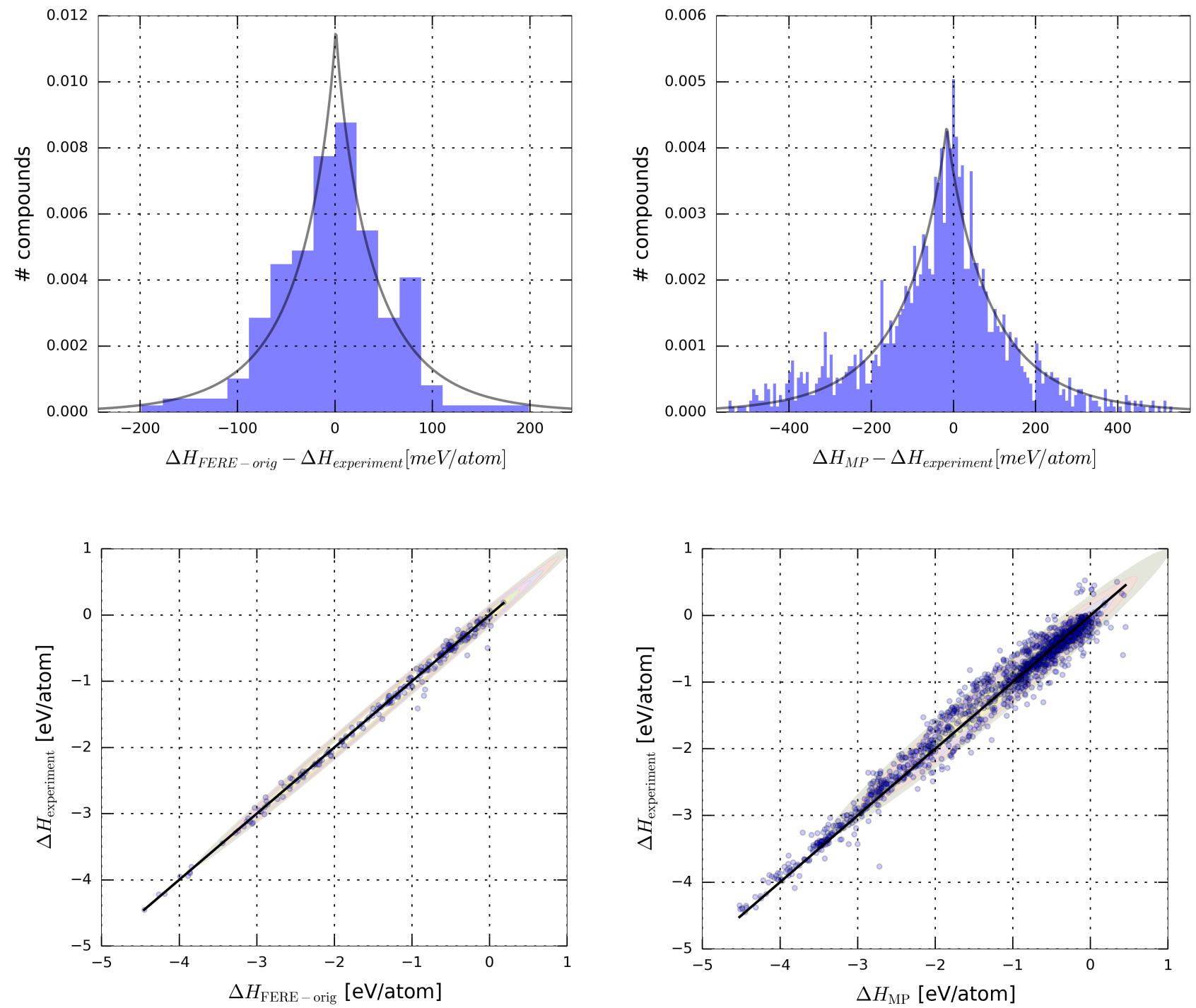

FIG. 15. Distribution of computational error for empirical correction schemes: FERE (left) and Materials Project corrected scheme (right). For FERE, the data comes from table II in Ref. 14. For the Material Project, the corresponding corrected GGA values were downloaded using their Python interface. Fitting of the distribution was done using the maximum likelihood method fit() (from Scipy ${ }^{111}$ ) for the generalized-normal distribution (scipy.stats.gennorm). The distributions are summarized in the third and fourth columns of table III.

Narayan et al. ${ }^{112}$ constructed a related statistical model to address theoretical predictions of new materials using formation energies computed with the Materials Project corrections. They proposed an optimal cutoff energy $\epsilon_{0}=0.1 \mathrm{eV}$, to minimize the rates of false-positives and false-negatives. They suggested that the synthesis of materials with $\epsilon<\epsilon_{0}$ should be attempted. Our analysis can be seen as a refinement of the idea of a cutoff.

From an experimental standpoint, the question to answer is whether an experimental search should be pursued or not. This depends not only on the function $\mathcal{P}(x)$ but also on how interesting is the estimated property that we are seeking. Also, notice that we are asking the question of whether we will obtain a material close to its ground state. It is well known that many known compounds are metastable $^{113}$. Therefore, when looking for compounds which have not been synthesized before, we are obtaining a lower bound for the probability of finding a new material, which is in greater than $\mathcal{P}(x)$. Third, notice that stability analyses based on convex hulls can only rule out the stability of a compound, rather than definitively prove its stability, since technically there are an infinite number of compositions and structures to consider in the phase diagram.

Theory assisted material discovery has already had notable successes, where the predicted compounds were successfully synthesized. It has lead to new multiferroic materials (Refs. 7 and 114). The material design strat- 


\begin{tabular}{c|c|c||c|c}
\hline & \multicolumn{2}{|c||}{ Before corrections } & \multicolumn{2}{c}{ After correction } \\
\cline { 2 - 5 } & GGA $(\mathrm{U}=0)$ & $\mathrm{MP}(+\mathrm{U})$ & FERE & MP \\
\hline Mean & 136 & 151 & 6 & -31 \\
\hline MAE & 155 & 172 & 51 & 130 \\
\hline$\sigma$ & 427 & 266 & 81 & 192 \\
\hline \hline center $\mu$ & - & - & 1 & -16 \\
\hline shape $\beta$ & - & - & 0.85 & 0.90 \\
\hline scale $\alpha$ & - & - & 39 & 109 \\
\hline \# data points & 1500 & 1598 & 227 & 1598 \\
\hline
\end{tabular}

TABLE III. Statistics for the distribution of error $\mathcal{F}$. Maximum likelihood values for the parameters of the distribution function in Eq. (30) are given for the correction schemes. All units are meV except for $\beta$ (unitless) and the number of points. MP $(+\mathrm{U})$ stands for Materials Project's raw data, which only includes the $\mathrm{U}$ correction for certain correlated compounds, as described in appendix.

egy was used to discover and synthesize 18 new ternary semiconductors $^{8}$. It has also been used to find two new structures in the Ce-Ir-In system ${ }^{9}$. There are also reports of broad theoretical searches where experimental synthesis did not find the theoretical approach predictive ${ }^{112}$. As an example from that work, we examine $\mathrm{KScS}_{2}$ which has $\mathcal{E}_{\text {calc }}=-0.136 \mathrm{eV}$ relative to the convex hull. According to our analysis it has probability 0.8 to exist and indeed it has been synthesized successfully in Ref. 115 .

\section{CASE STUDIES}

In the following, we illustrate the concepts of the previous section providing examples of the design of correlated materials. We limited ourselves to a few projects we are familiar with and omitted important areas, as for example the design of nuclear fuels ${ }^{7,116-120}$, where strong correlations in the solid state play a major role.

The examples encompass materials displaying superconductivity VI A, VIB, VID, charge disproportionation VIC, and metal-insulator transitions VIE.

The motivation for material design projects in the field of strongly correlated electron materials is not only to find new compounds, but to use the process to check our understanding of the theory, validating correct ideas and approaches and discarding wrong ones. In each case the material design project begins with some intuitive idea that one would like to test. We then move through the procedure outlined in section IV, describing the steps to go from structure to property, from composition to structure and from components to composition. We conclude each section with the lessons learned from the project. We highlight the tools currently in use and the role that correlations play in each stage of the workflow.

The field of material design of strongly correlated electron systems is in its infancy and the examples span the development of the workflow itself-in some cases in the examples, only part of the full workflow was applied in the design process. Throughout the presentation we stress the importance of qualitative ideas and chemical principles and how they can be supported and enhanced with modern computational techniques.

\section{A. Tuning the charge-transfer energy}

\section{Background}

The cuprate superconductors are classic examples of correlated materials. Since this family of compounds exhibits the highest known superconducting transition temperatures at ambient pressure (surpassed only by $\mathrm{H}_{3} \mathrm{~S}$ at high pressures), they have been intensely studied since their discovery in the mid-1980s. They have motivated an immense body of work in condensed matter physics, both in new theories and huge leaps in experiment.

Structurally, all the cuprate families have in common $\mathrm{CuO}_{2}$ planes which support superconductivity. They are described by the chemical formula $\mathrm{XS}_{n-1}\left(\mathrm{CuO}_{2}\right)_{n}$, where $n \mathrm{CuO}_{2}$ planes are interleaved with $n-1$ spacer layers $\mathrm{S}$ to form a multi-layer. These multi-layers are then stacked along the c-axis, separated by a different spacer layer $\mathrm{X}$. It is known that the critical temperature is a strong function of doping, and for each family there is an optimal doping where the maximum superconducting $T_{\mathrm{c}}\left(T_{\mathrm{c}, \max }\right)$ occurs. Empirically, it is known that $T_{\mathrm{c}, \max }$ is strongly materials-dependent, ranging from $40 \mathrm{~K}$ in $\mathrm{La}_{2} \mathrm{CuO}_{4}$ to $138 \mathrm{~K}$ in $\mathrm{HgBa}_{2} \mathrm{Ca}_{2} \mathrm{Cu}_{3} \mathrm{O}_{8}$.

It is now agreed that superconductivity arises from doping a parent compound which is a charge transfer antiferromagnetic insulator ${ }^{121}$, and that the symmetry of the superconducting state in these materials is $d$-wave, as it was predicted theoretically ${ }^{122-124}$. One should stress however, that there is no consensus on the mechanism that control the superconducting critical temperature and as a consequence what leads to the correlation of $T_{\mathrm{c}, \max }$ with the apical oxygen distance. Even if one accepts a mechanism, such as the proximity to magnetism and the presence of on-site Hubbard correlations, controversies persist, as both weak coupling and strong coupling approaches predict the correct nature of the superconductivity.

Setting the Targets \& Framing the Questions - Could materials design help us verify or falsify theories of the high-temperature cuprate superconductors? Or at least narrow down the set of competing theories and sharpen our theoretical understanding while suggesting new compounds in this important class of materials?

We chose the $T$-type layered perovskite $\mathrm{La}_{2} \mathrm{CuO}_{4}$ as the starting point. Our intuition led us to propose the site substitution of the apical oxygen with sulfur. Due to the larger ionic radius of sulfur as compared to oxygen we expect the LaS charge reservoir layer to be crowded. To compensate, we explored the effect of substituting the large La ion with smaller trivalent ions $R$, selected from the lanthanide-like elements. The compositions 


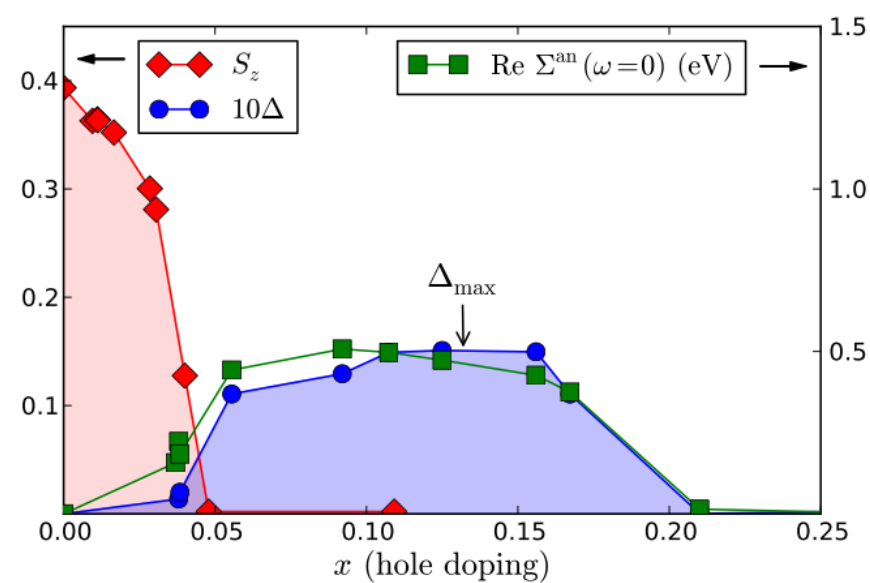

FIG. 16. Cluster DMFT investigation of a model of $\mathrm{La}_{2-x} \mathrm{Sr}_{x} \mathrm{CuO}_{4}$ (Weber et al. ${ }^{126}$ ). Zero temperature phase diagram connects structure to physical properties (superconductivity and antiferromagnetism).

we considered were $R_{2} \mathrm{CuS}_{2} \mathrm{O}_{2}$ (shown in Fig. 19) and $R_{2} \mathrm{CuSO}_{3}$. We include the monosulfide in hopes that the configurational entropy of only replacing a quarter of the apical oxygens with sulfur would help stabilize the target phase.

\section{Structure to Property}

Cluster DMFT can describe the competition and synergy between antiferromagnetism and $d$-wave superconductivity $^{125}$.

Weber et al. ${ }^{126}$ used Cluster LDA+DMFT with an exact diagonalization to bridge between the structure of $\mathrm{La}_{2-x} \mathrm{Sr}_{x} \mathrm{CuO}_{4}$ (LSCO) and the physical properties of magnetism and superconductivity. As shown in Fig. 16, the method describes well the zero temperature phase diagram of this compound, including the antiferromagnetic phase (characterized by the magnetic moment $S_{z}$ ) and the $d$-wave superconductor characterized by the anomalous self energy and the superconducting gap $\Delta$.

Multiple studies concluded that $T_{\mathrm{c}, \max }$ is an increasing function of the apical oxygen distance ${ }^{126-130}$. Changing the apical oxygen position, however, affects many different parameters of the electronic structure of the copper oxygen planes, ranging from the hoppings to the charge transfer energy and to the relative positions of the $d_{x^{2}-y^{2}}$ and $d_{z^{2}}$ orbitals. For a working design process, we need to elucidate the connection between these parameters and $T_{\mathrm{c}, \max }$.

To investigate the link between $T_{\mathrm{c}, \max }$ and the various parameters describing the electronic structure, one needs to vary them independently, which is easy to do in-silico, based on a combination of first principles calculations and dynamical mean-field theory to directly model the superconducting state. Specifically, $\mathrm{La}_{2-x} \mathrm{Sr}_{x} \mathrm{CuO}_{4}$ has the largest charge transfer energy and a small $T_{\mathrm{c}, \max }$, we vary the charge transfer energy and the hoppings independently and the results are shown in Fig. 18. It is clear that (within LDA+DMFT) the superconductivity increases rapidly when we decrease the charge transfer gap, while it is very weakly sensitive (and in fact decreases) when we increase the oxygen oxygen overlap and the hopping integral $t^{\prime}$ (depicted in Fig. 17) on the square lattice (not shown).

Inspired by these results and the earlier heuristic considerations of connecting structure to property, we aimed to design new cuprates with reduced charge transfer gaps (and thus higher $T_{c}$ ) via sulfur (S) substitution. To check that $\mathrm{S}$ substitution helps control the charge transfer gap, and to see which other variables it affects, we used the maximally localized Wannier function ${ }^{131,132}$ implemented in the WANNIER90 $\operatorname{code}^{133}$.

The results for the GGA estimates of the charge transfer energies are displayed in Fig. 19 and the second column of Table $\mathrm{V}$ together with the reference system $\mathrm{La}_{2} \mathrm{CuO}_{4}$. Both figure and table suggest that $\mathrm{Sc}_{2} \mathrm{CuS}_{2} \mathrm{O}_{2}$ having the highest charge transfer energy would have the smallest $T_{\mathrm{c}, \max }$ among proposed cuprates $R_{2} \mathrm{CuS}_{2} \mathrm{O}_{2}$ and $\mathrm{La}_{2} \mathrm{CuS}_{2} \mathrm{O}_{2}$ having smaller charge transfer energy than $\mathrm{La}_{2} \mathrm{CuO}_{4}$ would have higher $T_{\mathrm{c}, \text { max }}$ than that of $\mathrm{La}_{2} \mathrm{CuO}_{4}$ - based on the charge-transfer theory in Ref. 126.

Sakakibara et al. ${ }^{130}$ observed a positive correlation between $T_{\mathrm{c}, \max }$ and the crystal field splitting $\Delta E=$ $\epsilon_{d\left(x^{2}-y^{2}\right)}-\epsilon_{d z^{2}}$ based on a two-band model incorporating both $d_{x^{2}-y^{2}}$ and $d_{z^{2}}$ orbitals. They found that large crystal field splitting $\Delta E$, which is also related to the reduction of $d_{z^{2}}$ contribution to the Fermi surface, enhances $T_{\mathrm{c}, \max }$. In order to test their theory, we examine $\Delta E$ in Fig. 20 and the last column of Table V. The crystal field splitting $\Delta E$ in the oxysulfides family $R_{2} \mathrm{CuS}_{2} \mathrm{O}_{2}$ is generally smaller than that in $\mathrm{La}_{2} \mathrm{CuO}_{4}$, implying that sulfur substitution would suppress $T_{\text {c,max }} \cdot \mathrm{La}_{2} \mathrm{CuS}_{2} \mathrm{O}_{2}$ having the smallest crystal field splitting $\Delta E$ among the oxysulfides family, for example, has the smallest $T_{\mathrm{c}, \max }$ based on the theory, while the charge-transfer theory gives the opposite result. Therefore a detailed study of $\mathrm{La}_{2} \mathrm{CuS}_{2} \mathrm{O}_{2}$ is necessary to test the validity of these two theories.

Structure Prediction - To check for local stability we used a $2 \times 2 \times 1$ unit cell, we performed full structural relaxation to check if the structure would be unstable towards distortion to the $T^{\prime}$-type layered perovskite, knowing that substitution of the large La ion for the smaller $\mathrm{Pr}$ and $\mathrm{Nd}$ led to a rearrangement of the charge reservoir layer into the fluorite structure. We found that the $T$-type structure was indeed stable and there was no outof-plane buckling, although the $\mathrm{CuO}_{6}$ octahedra favored axial rotations $\left(a^{0} a^{0} c_{p}^{-}\right.$in Glazer notation). See Fig. 21.

Global stability - We checked the thermodynamic stability of the proposed compounds against competing phases by selecting commonly known reactants and computing the formation enthalpies of the synthesis pathways as shown in Table IV. We computed the total energies of formation $\Delta E=E_{\text {products }}-E_{\text {reactants, }}$, and find that all differentials are positive, indicating the re- 


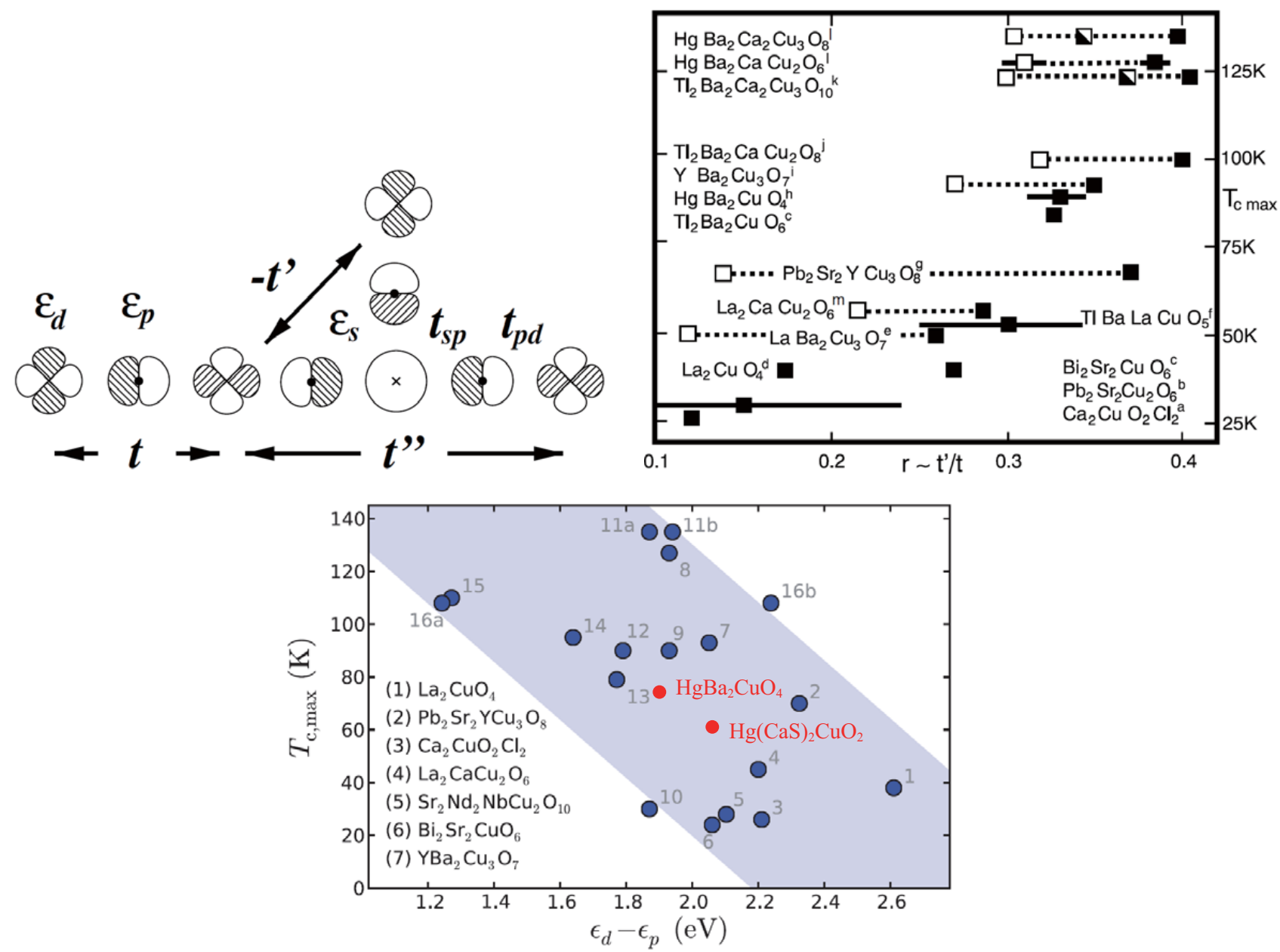

FIG. 17. The empirical correlation of $T_{c, \max }$ with the apical oxygen can be due to variation of many parameters in the low energy Hamiltonian describing different processes (top left), $t^{\prime} / t$ as originally suggested by Pavarini et al. ${ }^{127}$ (top right), as well as the charge transfer gap as suggested by Weber et al. ${ }^{126}$ (bottom). We have updated the bottom figure to include Hg-based cuprates.
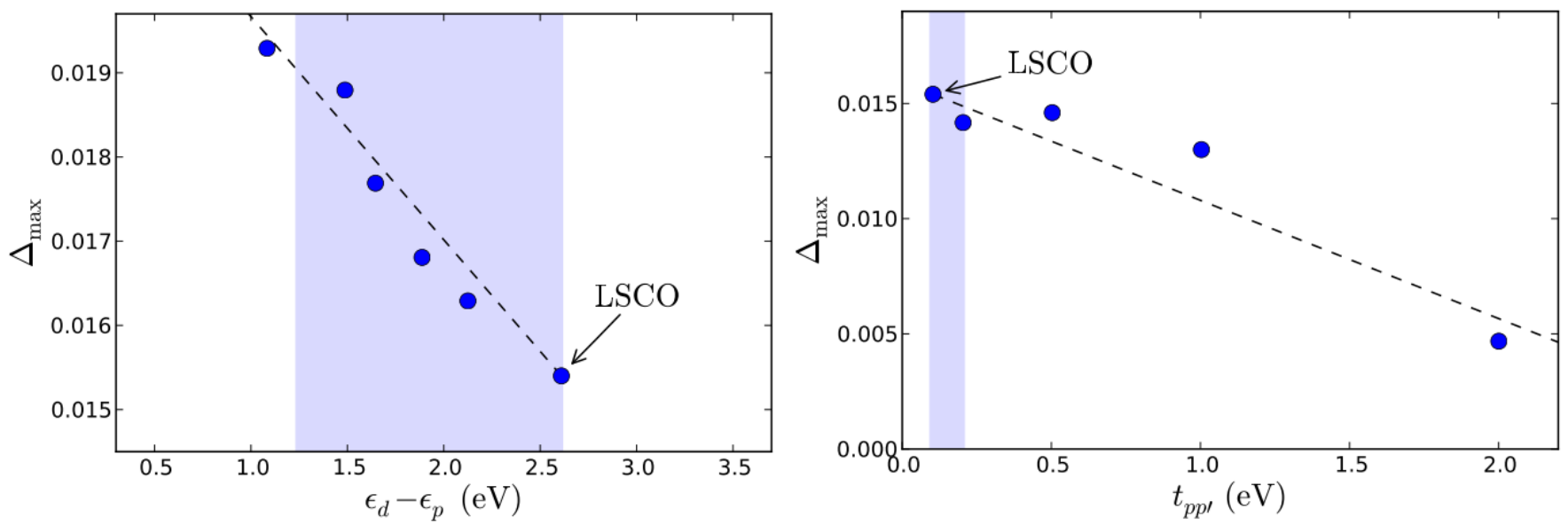

FIG. 18. Cluster DMFT investigation of a model of the copper oxide planes (Weber et al. ${ }^{126}$ ). After showing that the model displays the correct phase diagram (top), starting with $\mathrm{La}_{2-x} \mathrm{Sr}_{x} \mathrm{CuO}_{4}$ (LSCO) one varies independently the parameters that control the charge transfer energy $\epsilon_{p}-\epsilon_{d}$ (bottom left) and the oxygen oxygen overlap $t_{p p^{\prime}}$ (bottom right). The figure displays the dependence of the maximum of the superconducting order parameter (which serves as a proxy for $T_{c, \text { max }}$ ). We see that the maximum superconducting gap $\Delta_{\max }$ is very sensitive to the charge transfer gap, which thus controls (in this strong coupling theory) the critical temperature and not with $t^{\prime}$ or $t_{p p}^{\prime}$. 


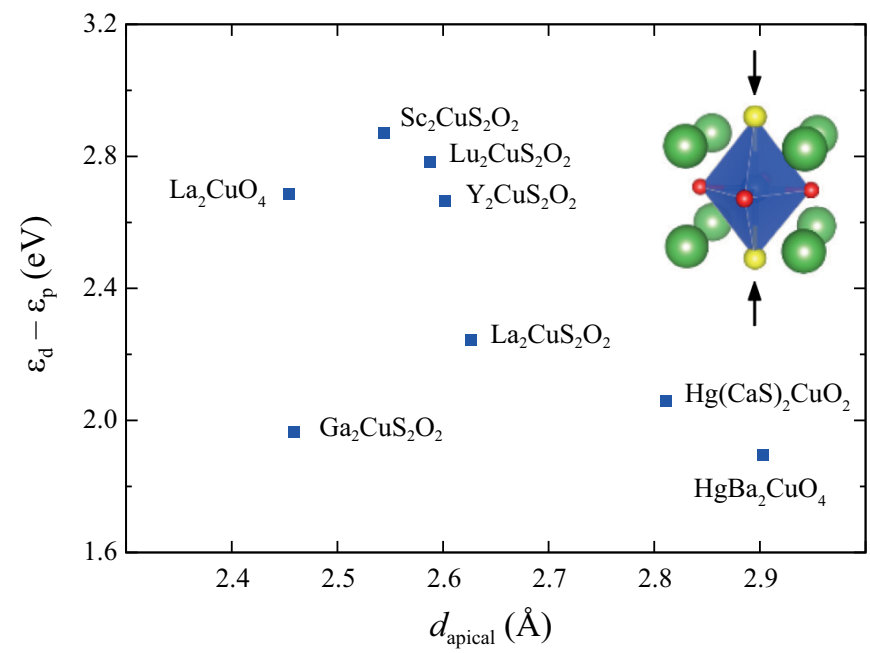

FIG. 19. Explored compositions, generated by substituting the apical oxygens (arrows) and rare earth ion (green spheres) in $\mathrm{La}_{2} \mathrm{CuO}_{4}$ to form the family of compounds $R_{2} \mathrm{CuS}_{2} \mathrm{O}_{2}$. Two mercury compounds $\mathrm{HgBa}_{2} \mathrm{CuO}_{4}$ and $\mathrm{Hg}(\mathrm{CaS})_{2} \mathrm{CuO}_{2}$ are also added for comparison. Plotted are the extracted charge-transfer energies vs. the apical distance of sulfur atom in the proposed compounds after structural relaxation in LDA. Note that charge-transfer energies in known cuprates superconductors are observed in the range from $\sim 1.2$ to $\sim 2.6$ eV. Adapted from Ref. 134.

actions target phases are unfavorable. However, it is known that many functional materials are metastable, protected from decay by large energetic barriers. The parent cuprate $\mathrm{La}_{2} \mathrm{CuO}_{4}$ is an example: as shown on the last line of Table IV, $\mathrm{La}_{2} \mathrm{CuO}_{4}$ is actually unstable by $65 \mathrm{meV} /$ atom. We also examined the volume differentials $\Delta V=V_{\text {products }}-V_{\text {reactants }}$ with the knowledge that often high pressure synthesis allows otherwise unstable compounds to form. Notice that $\Delta V$ are overwhelming negative, meaning the application of high pressure may allow the formation of the target phases.

To look at the question of global stability of $\mathrm{La}_{2} \mathrm{CuS}_{2} \mathrm{O}_{2}$ and $\mathrm{La}_{2} \mathrm{CuSO}_{3}$, we use the modern materials databases, and reanalyze the entire $\mathrm{La}-\mathrm{Cu}-\mathrm{S}-\mathrm{O}$ system to construct the convex hull (plotted in Fig. 22) and globally investigate stability.

Using the convex hull, we can assess the stability of the reactants and products reported in experiment. In Fig. 23, we plot the energies relative to the convex hull for all reported compounds. Negative values are stability energies against decomposition. We find that $\mathrm{La}_{2} \mathrm{CuS}_{2} \mathrm{O}_{2}$ and $\mathrm{La}_{2} \mathrm{CuSO}_{3}$ are unstable at 232 and $324 \mathrm{meV}$ /atom above the hull, respectively, which is slightly more unstable than the earlier estimates which only tested a few reactions, and we gain additional information as we learn these compounds in equilibrium would decompose into:

$$
\begin{aligned}
& \mathrm{La}_{2} \mathrm{CuS}_{2} \mathrm{O}_{2} \rightarrow \mathrm{La}_{2} \mathrm{SO}_{2}+\mathrm{CuS} \\
& 4 \mathrm{La}_{2} \mathrm{CuSO}_{3} \rightarrow 3 \mathrm{La}_{2} \mathrm{SO}_{2}+4 \mathrm{Cu}+\mathrm{La}_{2} \mathrm{SO}_{6}
\end{aligned}
$$

Additionally, LaCuSO lies $17 \mathrm{meV} /$ atom below the

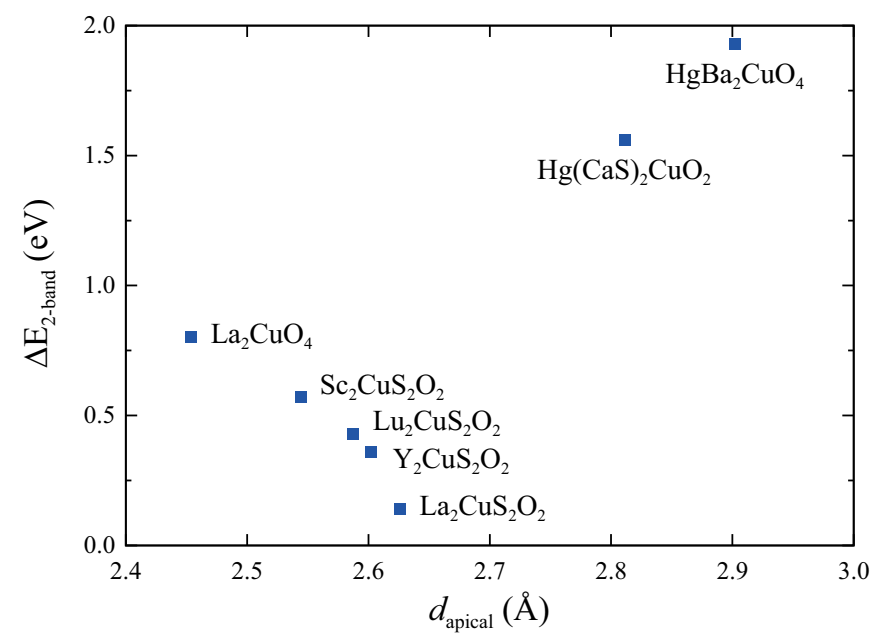

FIG. 20. The crystal field splitting $\triangle E_{2-\text { band }}=\epsilon_{d\left(x^{2}-y^{2}\right)}-$ $\epsilon_{d z^{2}}$ computed by downfolding into the effective two-band model. $\mathrm{La}_{2} \mathrm{CuO}_{4}$ and the copper oxysulfide family are shwon with two mercury compounds $\mathrm{HgBa}_{2} \mathrm{CuO}_{4}$ and $\mathrm{Hg}(\mathrm{CaS})_{2} \mathrm{CuO}_{2}$ for comparison. $\triangle E_{2-\text { band }}$ in the oxysulfides is generally smaller, implying stronger mixing between $d_{z^{2}}$ and $d_{x^{2}-y^{2}}$ orbitals. Thus sulfur substitution would suppress $T_{\mathrm{c}, \max }$ according to the two-band theory ${ }^{130}$, while $T_{\mathrm{c}, \max }$ would be enhanced according to the charge-transfer theory ${ }^{126}$. Adapted from Ref. 134.

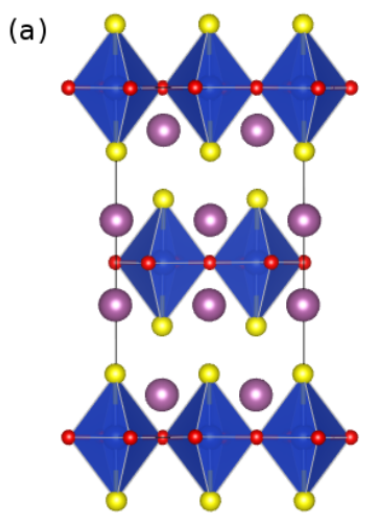

(b)

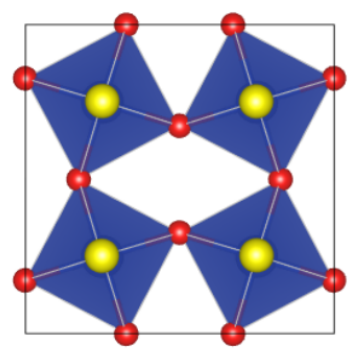

FIG. 21. Octahedral rotations in $\mathrm{Sc}_{2} \mathrm{CuS}_{2} \mathrm{O}_{2}$ as shown by a section of the $\mathrm{CuO}_{2}$ plane with four atoms. Adapted from Ref. 134.

hull, so it is likely to be stable according to the analysis of section $\mathrm{V}$.

Following our proposal in section $\mathrm{V}$, we obtain sharper probability estimates using a correlated method - GGA(PBE) + Gutzwiller. As outlined, we only need to examine the energies of the determinant reaction. The on-site Coulomb interaction $U=8 \mathrm{eV}$ and Hund's coupling constant $J=0.88 \mathrm{eV}$ are used in the Gutzwiller calculations ${ }^{135}$. We find that $\mathrm{La}_{2} \mathrm{CuS}_{2} \mathrm{O}_{2}$ and $\mathrm{La}_{2} \mathrm{CuSO}_{3}$ are still unstable at 214 and $286 \mathrm{meV} /$ atom above the hull, respectively - consistent with a recent experiment ${ }^{136}$.

Conclusion - Experimental support for the idea that the reduction of the charge transfer gap results in an 


\begin{tabular}{r|c|cc}
\hline$\Delta E$ & $\Delta V$ & \multicolumn{2}{|c}{ Synthesis pathway } \\
\hline \hline 232 & -1.92 & $\mathrm{La}_{2} \mathrm{SO}_{2}+\mathrm{CuS}$ & $\rightarrow \mathrm{La}_{2} \mathrm{CuS}_{2} \mathrm{O}_{2}$ \\
362 & -1.45 & $\mathrm{Y}_{2} \mathrm{SO}_{2}+\mathrm{CuS}$ & $\rightarrow \mathrm{Y}_{2} \mathrm{CuS}_{2} \mathrm{O}_{2}$ \\
415 & -1.36 & $\mathrm{Lu}_{2} \mathrm{SO}_{2}+\mathrm{CuS}$ & $\rightarrow \mathrm{Lu}_{2} \mathrm{CuS}_{2} \mathrm{O}_{2}$ \\
542 & -1.04 & $\mathrm{Sc}_{2} \mathrm{SO}_{2}+\mathrm{CuS}$ & $\rightarrow \mathrm{Sc}_{2} \mathrm{CuS}_{2} \mathrm{O}_{2}$ \\
\hline 304 & -2.00 & $\mathrm{La}_{2} \mathrm{O}_{3}+\mathrm{CuS}$ & $\rightarrow \mathrm{La}_{2} \mathrm{CuSO}_{3}$ \\
780 & -1.11 & $\mathrm{Sc}_{2} \mathrm{O}_{3}+\mathrm{CuS}$ & $\rightarrow \mathrm{Sc}_{2} \mathrm{CuSO}_{3}$ \\
217 & -1.28 & $\mathrm{La}_{2} \mathrm{SO}_{2}+\mathrm{CuO}$ & $\rightarrow \mathrm{La}_{2} \mathrm{CuSO}_{3}$ \\
519 & -0.54 & $\mathrm{Sc}_{2} \mathrm{SO}_{2}+\mathrm{CuO}$ & $\rightarrow \mathrm{Sc}_{2} \mathrm{CuSO}_{3}$ \\
\hline 65 & -2.71 & $\mathrm{LaCuO}_{2}+2 \mathrm{La}_{2} \mathrm{O}_{3}+\mathrm{La}\left(\mathrm{CuO}_{2}\right)_{2}$ & $\rightarrow 3 \mathrm{La}_{2} \mathrm{CuO}_{4}$ \\
\hline
\end{tabular}

TABLE IV. Synthesis pathways for various cuprate oxysulfides based on substitution of sulfur for both (top block) or only one (middle block) of the apical oxygens in $R_{2} \mathrm{CuO}_{4}$. Energies in $\mathrm{meV} /$ atom and volumes in $\AA^{3}$ /atom. Since the energies of formation $\left(\Delta E=E_{\text {products }}-E_{\text {reactants }}\right)$ are positive, none of these pathways appear favorable at ambient conditions. However, high-pressure synthesis will help stabilize these pathways, since the majority of volume differentials $\left(\Delta V=V_{\text {products }}-V_{\text {reactants }}\right)$ are negative. We benchmark our method against the standard synthesis pathway for $\mathrm{La}_{2} \mathrm{CuO}_{4}$, shown on the last line. Surprisingly, $\Delta E$ is $+65 \mathrm{meV} /$ atom, so either DFT systemmatically overestimates enthalpies (which means the actual enthalpies for our hypothetical compounds are smaller, in our favor), or we must add a bi-directional uncertainty of $\pm 70 \mathrm{meV} /$ atom to the computed enthalpies. Additionally, positional entropy of the apical $S$ in the halfsubstituted $R_{2} \mathrm{CuSO}_{3}$ compounds should also assist in synthesis.

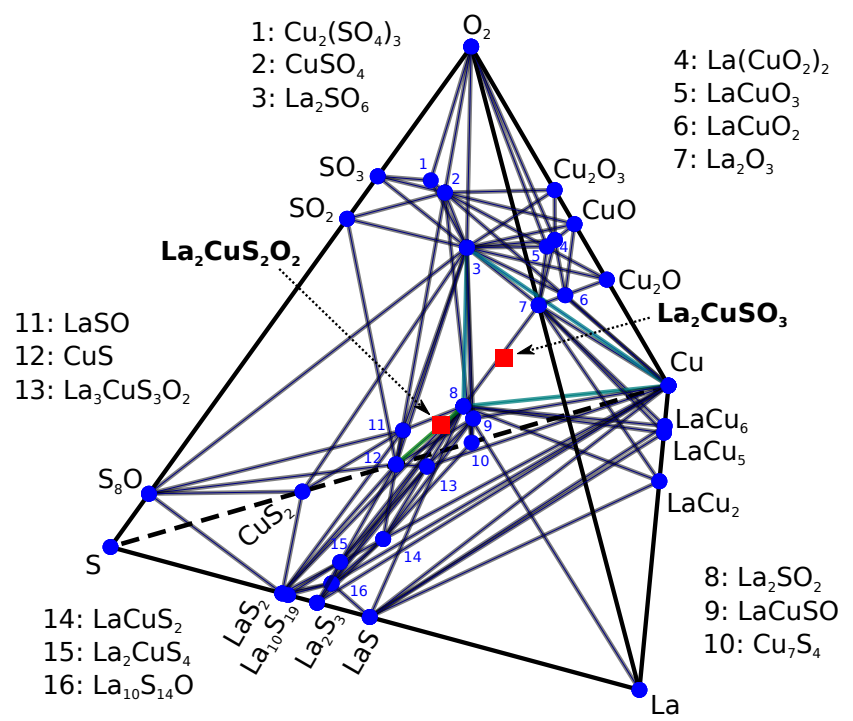

FIG. 22. Gibbs phase diagram of La-Cu-S-O system. The two proposed compositions are marked by red squares. They are both found to be unstable: $\mathrm{La}_{2} \mathrm{CuS}_{2} \mathrm{O}_{2}$ lies on the line between $\mathrm{CuS}(12)$ and $\mathrm{La}_{2} \mathrm{SO}_{2}(8)$, and $\mathrm{La}_{2} \mathrm{CuSO}_{3}$ lies on the triangular facet spanned by $\mathrm{Cu}, \mathrm{La}_{2} \mathrm{SO}_{2}(8)$, and $\mathrm{La}_{2} \mathrm{SO}_{6}(6)$. In experiment, we find that the quaternary phase LaCuSO (9) is preferred, likely because copper prefers the $1+$ oxidation state.

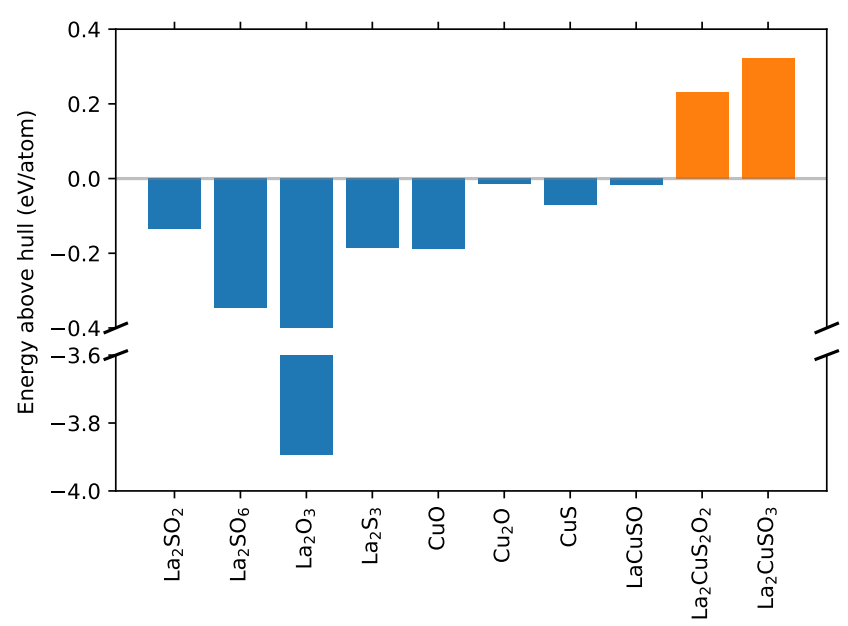

FIG. 23. Energies relative to the convex hull for reactants and products observed in experiment in the La-Cu-S-O system. Negative energies indicate stable compounds, while unstable compounds have positive energies. Both $\mathrm{La}_{2} \mathrm{CuSO}_{3}$ and $\mathrm{La}_{2} \mathrm{CuS}_{2} \mathrm{O}_{2}$ are highly unstable, lying over $200 \mathrm{meV} /$ atom above the hull. The vertical axis is broken to display the large stability energy of $\mathrm{La}_{2} \mathrm{O}_{3}$.

increase in $T_{\mathrm{c}}$ were recently provided by STM studies which were used to compare the charge transfer gaps of $\mathrm{Ca}_{n+1} \mathrm{Cu}_{n} \mathrm{O}_{2 n} \mathrm{Cl}_{2}$ and $\mathrm{Bi}_{2} \mathrm{Sr}_{2} \mathrm{Ca}_{n-1} \mathrm{Cu}_{n} \mathrm{O}_{2 n+4}{ }^{137}$, hence the ideas proposed in Ref. 126 are definitely worth pursuing. It is not known however, where exactly the maximum of $T_{\mathrm{c}}$ is obtained, as it is clear that a very small charge transfer energy would result in an uncorrelated material with very low $T_{\mathrm{c}}$. So, while this problem remains open, we can already say that the work of Ref. 126 has served to provide context for later experimental work.

Synthesis performed at synchrotron light sources now allows for in situ tracking of intermediate products and provides a deep understanding of the chemical reaction pathways by which a compound forms at different temperatures $^{138,139}$. The group of M. Aronson used this technique to study the system described in this section ${ }^{136}$ and investigated the potential synthesis of $\mathrm{La}_{2} \mathrm{CuS}_{2} \mathrm{O}_{2}$ and $\mathrm{La}_{2} \mathrm{CuSO}_{3}$. Their work confirmed that the two compounds were unstable (at least at high temperatures). From the convex hull (Fig. 22), the target compounds are thermodynamically unstable with respect to $\mathrm{CuS}$, $\mathrm{La}_{2} \mathrm{SO}_{2}, \mathrm{Cu}$, and $\mathrm{La}_{2} \mathrm{SO}_{6}$ and the phase with composition LaCuSO seemed to be quite stable at high temperatures. In agreement with theory, the end products $\mathrm{La}_{2} \mathrm{SO}_{2}$ and $\mathrm{La}_{2} \mathrm{SO}_{6}$ were observed, in addition to La$\mathrm{CuSO}$ which was the preferred quaternary composition in almost all the experimentally analyzed reactions. This experimental study thus validates in detail the current material design workflow and its probabilistic interpretation.

Sulfate apical substitution is not easily realized but the work of Ref. 126 has served to provide context for later experimental work ${ }^{136}$, which highlight the difficulties in forming the desired oxysulfides. The more flexible 


\begin{tabular}{|c|c|c|c|c|c|}
\hline & $\Delta_{p d}(\mathrm{eV})$ & $\Delta E(\mathrm{eV})$ & $E_{g}(\mathrm{eV})$ & $S_{z}\left(\mu_{B} / \mathrm{Cu}\right)$ & $\Delta E_{2-\text { band }}(\mathrm{eV})$ \\
\hline \hline $\mathrm{La}_{2} \mathrm{CuO}_{4}$ & 2.69 & 0.03 & 2.01 & 0.71 & 0.80 \\
\hline $\mathrm{La}_{2} \mathrm{CuS}_{2} \mathrm{O}_{2}$ & 2.24 & 0.09 & 1.47 & 0.72 & 0.14 \\
\hline $\mathrm{Sc}_{2} \mathrm{CuS}_{2} \mathrm{O}_{2}$ & 2.87 & 0.35 & 0.99 & 0.70 & 0.57 \\
\hline $\mathrm{Lu}_{2} \mathrm{CuS}_{2} \mathrm{O}_{2}$ & 2.78 & 0.23 & 1.67 & 0.71 & 0.43 \\
\hline $\mathrm{Y}_{2} \mathrm{CuS}_{2} \mathrm{O}_{2}$ & 2.66 & 0.19 & 1.48 & 0.71 & 0.36 \\
\hline $\mathrm{HgBa}_{2} \mathrm{CuO}_{4}$ & 1.90 & 0.29 & 1.12 & 0.68 & 1.93 \\
\hline $\mathrm{Hg}(\mathrm{CaS})_{2} \mathrm{CuO}_{2}$ & 2.06 & 0.24 & 1.73 & 0.69 & 1.56 \\
\hline
\end{tabular}

TABLE V. Physical parameters for cuprate materials. The charge transfer energy $\Delta_{p d}=\epsilon_{d}-\epsilon_{p}$ and crystal field splitting $\Delta E=\epsilon_{d\left(x^{2}-y^{2}\right)}-\epsilon_{d z^{2}}$ are obtained from the Wannier method applied to GGA(PBE) nonmagnetic calculations. All of the Cu $d, \mathrm{O} p$, and S $p$ orbitals are considered for the Wannier method. The band gap $E_{g}$ and magnetic moment $S_{z}$ (per Cu atom) are caluclated within GGA(PBE) + U antiferromagnetic calculations. The on-site Coulomb interaction $U=8 \mathrm{eV}$ and Hund's coupling constant $J=0.88 \mathrm{eV}$ are used in the GGA+U calculations ${ }^{135}$. The last column of the table describes the results for the crystal field splitting downfolding to a two band model describing the $d_{x^{2}-y^{2}}$ and the $d_{z^{2}}$ in order to test the theory of Ref. 130.

valence of sulfur which can now reduce copper from its starting $2+$ oxidation state to bring it to its $1+$ state, and thus forms LaCuSO instead of our target compounds, which like $\mathrm{La}_{2} \mathrm{CuO}_{4}$, requires a $2+$ copper state ${ }^{136}$. With this insight we can now go back to the first step of the design loop to search for better routes to reduce the charge transfer gap while keeping the valence of $\mathrm{Cu}$ close to $2+$ or turn to other routes to vary the charge transfer energy as described in the next section.

\section{B. Hg-based Cuprates}

With the experience of the previous section we revisit the question of how to design novel cuprates following Ref. 140. The challenge in the design process is finding a new chemical composition that forms in the desired cuprate structure - with slightly different parameters, thus enabling the elucidation of the mechanism of superconductivity. We focus on the charge transfer energy ${ }^{126}$. We also consider the $d_{z^{2}}$ admixture which is the key variable in the orbital distillation theory ${ }^{130}$. This theory is supported by weak coupling calculations that show that a reduction in the content of $d_{z^{2}}$ (which correlates with an increase of the crystal field splitting $\Delta E$ ) increases the superconducting critical temperature.

Design of novel cuprates is challenging because the phase space is well-explored: most simple point substitutions likely have been attempted. On the other hand, an exhaustive search consisting of structure prediction over all possible compositions containing copper would be prohibitively costly. Rather than designing novel cuprates from scratch, we took an intermediate route: begin with the family with the highest known transition temperatures, the Hg-based cuprates, and modulate its spacer layers ${ }^{140}$.

Setting the Targets, Framing the Questions, Heuristic Considerations - Taking the Hg-based cuprates as an example (Fig. 24), we view the cuprates as a stack of functional layers, with the composition of each layer chosen to play a specific role. The central copper oxide $\left(\mathrm{CuO}_{2}\right)$ plane supports superconductivity and roughly constrains the in-plane lattice constant. The remaining layers must tune the chemical potential of the $\mathrm{CuO}_{2}$ layer without rumpling the plane or introducing disorder, and isolate each $\mathrm{CuO}_{2}$ plane to create a $2 \mathrm{D}$ system.

Our goal is to tune the in-plane charge transfer energy (effective $U$ ), so the relevant layers to focus on are the $\mathrm{BaO}$ layers immediately adjacent to the $\mathrm{CuO}_{2}$ plane. Due to their spatial proximity, the $\mathrm{BaO}$ layers tune the hoppings and interaction strengths of the in-plane Hamiltonian. We also pay attention to the energy of the $d_{z^{2}}$ orbital, as it plays a key role in the orbital distillation theory ${ }^{130}$.

Designing compounds with novel adjacent layers provides a mechanism for controlling superconductivity. However, cycling through all roughly $100 \times 100$ elemental substitutions for $\mathrm{BaO}$ in the periodic table using structural prediction is clearly too naive and computationally expensive. To select plausible compositions, we noted that the $\mathrm{BaO}$ layers form a rock salt structure. Using materials databases, we selected all naturally occurring rock salt compounds AX, composed of a cation A and an anion $\mathrm{X}$, starting with 333 in total. We then quickly prescreened candidates by discarding compositions with (1) large lattice mismatches relative to the in-plane $\mathrm{Cu}-\mathrm{Cu}$ distance, which we took to be $3.82 \AA$, and (2) anions less electronegative than $\mathrm{Cu}$, as these anions would capture dopants intended for the superconducting plane, producing additional Fermi surfaces.

Electronic structure - To evaluate the prospects of superconductivity, we follow the previous section, and we focus on the charge transfer gap. This is summarized in additional entries in Table $\mathrm{V}$. We find the charge-transfer energy of $\mathrm{Hg}(\mathrm{CaS})_{2} \mathrm{CuO}_{2}$ (HCSCO) to be $2.06 \mathrm{eV}$. It is slightly larger than other $\mathrm{Hg}$-based cuprate, $\mathrm{HgBa}_{2} \mathrm{CuO}_{4}$ ( $\mathrm{HBCO}$ ), however is smaller than $\mathrm{La}_{2} \mathrm{CuO}_{4}$ and the proposed cuprates $R_{2} \mathrm{CuO}_{2} \mathrm{~S}_{2}$ in Table $\mathrm{V}$. Hence we expect $T_{\mathrm{c}, \text { max }}$ of HCSCO is smaller than that of $\mathrm{HBCO}$, but is larger than those of $\mathrm{La}_{2} \mathrm{CuO}_{4}$ and $R_{2} \mathrm{CuS}_{2} \mathrm{O}_{2}$ (see the 


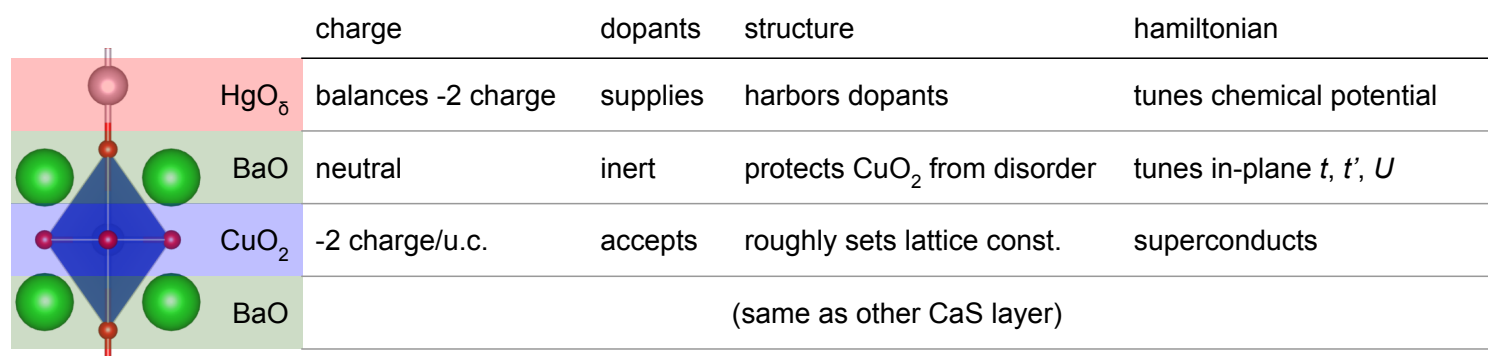

FIG. 24. The cuprates are a heterostructure of functional layers, each performing a specific structural and electronic role in tuning the superconducting hamiltonian. Here, we show the example of $\mathrm{HgBa}_{2} \mathrm{CuO}_{4+\delta}$, the single layer cuprate with the highest transition temperature. In our work, we focus on tuning the chemistry of the layers immediately adjacent to the $\mathrm{CuO}_{2}$ plane, as these will most strongly affect the in-plane Hamiltonian. Adapted from Ref. 140.

bottom of Fig. 17).

It is also useful to plot the orbitally-resolved band structure (Fig. 26). There is indeed a single band crossing the Fermi level in HCSCO, similar to the other cuprates.

Structural Stability - We tested to check that the desired structure was stable by first point-substituting the proposed elements into the $\mathrm{HgBa}_{2} \mathrm{CuO}_{4}$ (HBCO) structure and checked for local stability via phonon calculations at the $\Gamma,(\pi, 0)$ and $(\pi, \pi)$ points. If the composition was stable in the HBCO structure, then we used USPEX to perform the roughly week-long calculations necessary to determine whether the HBCO structure is indeed the preferred energetic minimum.

We found three $\mathrm{BaO}$ substitutions to be stable in the HBCO structure after phonon screening: $\mathrm{CaS}, \mathrm{ZrAs}$, and YbS. USPEX found that only $\mathrm{Hg}(\mathrm{CaS})_{2} \mathrm{CuO}_{2}$ (HCSCO) was energetically stable in the layered cuprate structure.

Global stability - We construct the convex hull by computing the energies of all known compounds in the $\mathrm{Hg}$ $\mathrm{Ca}-\mathrm{Cu}-\mathrm{S}-\mathrm{O}$ system, which would produce a 4-dimensional tetrahedron. In reality, synthesis is performed in an oxygen environment parameterized by the chemical potential $\mu\left(\mathrm{O}_{2}\right)$. We find that for all values of $\mu\left(\mathrm{O}_{2}\right)$, HCSCO is unstable, so we pick the value for which the compound is closest to the convex hull and plot the results in Fig. 25.

Prior to examining the global stability of HCSCO, we benchmarked the methodology on $\mathrm{HgBa}_{2} \mathrm{CuO}_{4}$ itself. We find that at fixed oxygen stoichiometry, HBCO lies $74 \mathrm{meV} /$ atom above the hull. Upon modeling the doped compound using via a $3 \times 3$ supercell calculation ( $11 \%$ doping), we find $\mathrm{HBCO}$ to be even more unstable at $130 \mathrm{meV} /$ atom above the hull. As noted above, $\mathrm{La}_{2} \mathrm{CuO}_{4}$, the metastable structure theoretically predicted to be $65 \mathrm{meV}$ /atom above the hull does indeed exist.

We found that HCSCO lies $170 \mathrm{meV} /$ atom above the hull (existence probability: 0.09) at fixed oxygen stoichiometry, and $240 \mathrm{meV} /$ atom above the hull (existence probability: 0.05) for fixed oxygen chemical potential ${ }^{141}$, which means the compound is likely unstable, but not out of the realm of possibility for successful synthesis.

Revising the phase stability calculation - using a more accurate correlated method, as suggested in section $\mathrm{V}$, we

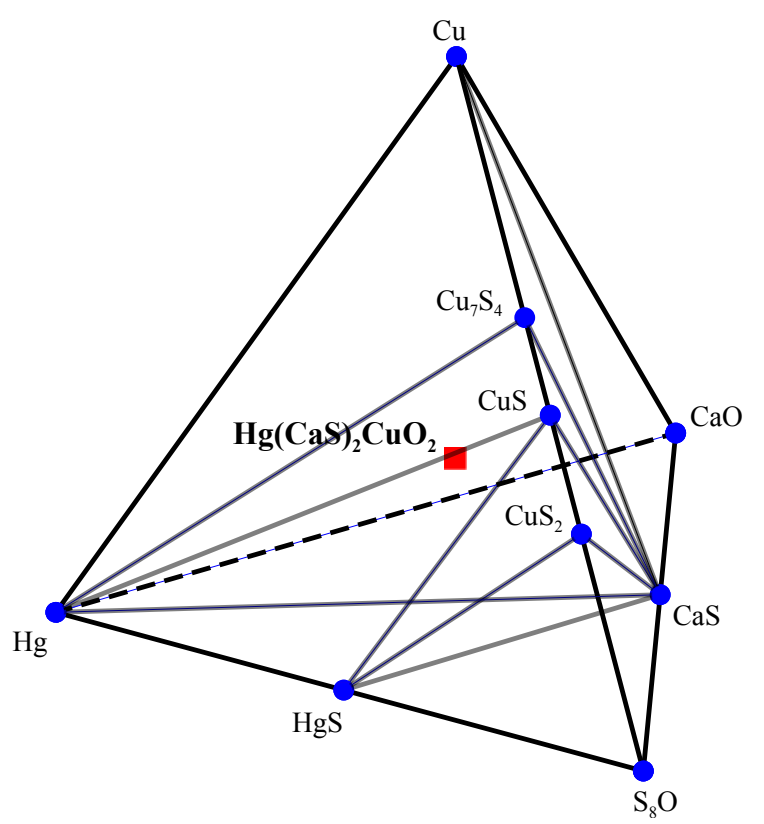

FIG. 25. Convex hull for the $\mathrm{Hg}-\mathrm{Ca}-\mathrm{Cu}-\mathrm{S}$ chemical system at an oxygen chemical potential of $\mu\left(\mathrm{O}_{2}\right)=-16.48 \mathrm{eV}$, chosen because HCSCO is least unstable at this value. The phase diagram forms a tetrahedron with $\mathrm{S}_{8} \mathrm{O}, \mathrm{Hg}, \mathrm{Cu}$ and $\mathrm{CaO}$ at the vertices (elemental sulfur and calcium are not stable under this oxygen environment). HCSCO lies in the interior of the tetrahedron, on the triangular face formed by $\mathrm{Hg}, \mathrm{Cu}$ and CaS.

consider the determinant reaction. This reaction is found by GGA to be $\mathrm{HgO}+2 \mathrm{CaS}+\mathrm{CuO} \rightarrow \mathrm{Hg}(\mathrm{CaS})_{2} \mathrm{CuO}_{2}$. Using the GGA(PBE) + Gutzwiller method to calculate the energies of the reactants, we found that HCSCO is stable with $151 \mathrm{meV} /$ atom below the hull (at fixed oxygen stoichiometry).

Conclusions - This work in modulating the spacer layers in the Hg-based cuprates highlights the challenges in optimizing properties in highly-explored materials classes. In the prediction of structures for novel phases, chemical intuition is still crucial for filtering possible candidates and focusing on the most promising compositions. Structure prediction is the bottleneck step in 


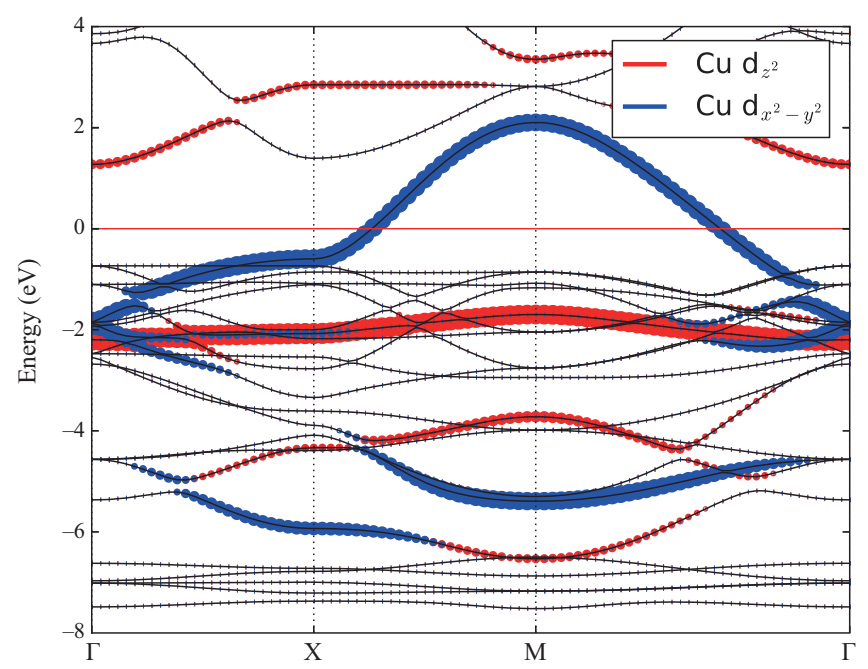

FIG. 26. Computed band structure of the proposed compound $\mathrm{Hg}(\mathrm{CaS})_{2} \mathrm{CuO}_{2}$ using $\mathrm{GGA}(\mathrm{PBE})$ nonmagnetic calculation. $\mathrm{Cu} 3 d_{z^{2}}$ and $3 d_{x^{2}-y^{2}}$ orbital characters are weighted by red and blue filled-circles, respectively. Similar to the cuprates, a single $\mathrm{Cu} 3 d_{x^{2}-y^{2}}$ band disperses across the Fermi level.

the workflow, consuming the largest fraction of the computational resources required. Additionally, known materials can be metastable, and new work must explore what factors select for which metastable compounds form in experiment. It would therefore be interesting to pursue the MBE route to approach the synthesis of this type of compound. Further searches which results in similar structures while avoiding the use of toxic chemicals such as $\mathrm{Hg}$ are also very important.

Theoretical calculations reproduced the early observation of Raychaudhury et al. ${ }^{142}$, that one can correlate $T_{c}$ with the oxygen-oxygen overlap and the next-nearestneighbor hopping parameter of the Hubbard model. Other correlations were pointed out - in particular that decreasing the charge transfer gap in these materials also increases $T_{c}{ }^{126}$. Finally, the orbital distillation proposal argues that a large admixture of the apical orbitals, in particular the $\mathrm{Cu}-d_{z^{2}}$ orbital, into the $d_{x^{2}-y^{2}}$ band, suppresses $T_{c}{ }^{130}$. It has motivated spectroscopic efforts to determine the degree of orbital distillation using ARPES $^{143}$ and STM measurements to map the chargetransfer gaps ${ }^{137,144}$.

Finding materials where the admixture of $\mathrm{Cu}-d_{z^{2}}$ orbital increases with the smaller charge-transfer gap would greatly advance our understanding of the mechanism of high temperature superconductivity in the cuprates, and as discussed in section VIA, it remains an outstanding challenge in material design.

Exploring the charge-transfer energy (Refs. 126, 145) dependence of $T_{\mathrm{c}}$ is worth pursuing further . There are several outstanding problems. There should be an optimal charge transfer energy to enhance $T_{\mathrm{c}}$, as it is clear that a very small charge transfer energy would result in an uncorrelated metal with very low $T_{\mathrm{c}}$. It is not known

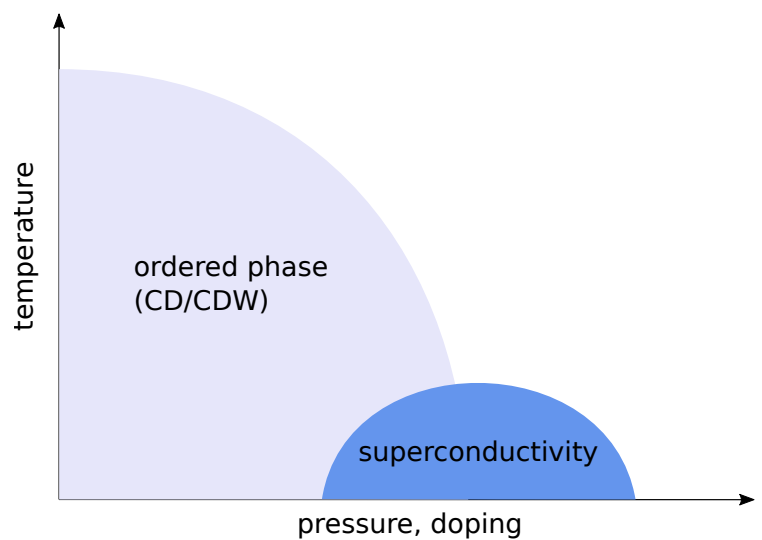

FIG. 27. Schematic phase diagram of the potassium-doped $\mathrm{BaBiO}_{3}$ system, which is representative of correlated materials as a whole. The phase diagram of correlated systems generally contain an ordered "parent" phase. This parent phase can be suppressed as a function of external parameters like pressure or doping, and superconductivity can arise near the phase transition.

however, where exactly the maximum of $T_{\mathrm{c}}$ is obtained, as it is clear that a very small charge transfer energy would result in an uncorrelated material with very low $T_{\mathrm{c}}$. Furthermore, taking the results of this section together with the previous section, it seems that the reduction of the charge transfer energy makes the formation of the compound more difficult (i.e. increases its distance from the convex hull). Once again, what are the limits which can be realized chemically, i.e. how much can the charge transfer gap be reduced while maintaining a stable compound - is a another interesting question.

\section{Valence Disproportionation in Other High Temperature Superconductors: $\mathrm{CsTlCl}_{3}$}

Motivation. - In our next example, we seek to design a parent compound for a new superconductor by creating a correlated material with valence disproportionation and strong electron phonon coupling. The hypothesis guiding the project, is that upon suppression of the charge/valence order by some means (doping, pressure, disorder), pairing will become the dominant instability and a dome of superconductivity will emerge. This guiding principle is ubiquitous in correlated materials: superconductivity generally appears upon suppression of an ordered "parent" phase as a function of experimental tuning parameters (see the prototypical phase diagram shown in Fig. 27). For a recent realization of this idea see Ref. 146 .

There are cases where the superconducting transition temperatures turn out to be well above those expected from the Migdal Eliashberg theory with the electron phonon coupling evaluated within LDA. These materials were dubbed the "other" high temperature superconductors $^{53,147}$.

A concrete realization of these principles is provided 
by $\mathrm{Ba}_{1-x} \mathrm{~K}_{x} \mathrm{BiO}_{3}$, a well-known superconducting system discovered in the $1980 \mathrm{~s}^{148,149}$. The parent compound is $\mathrm{BaBiO}_{3}$, a $\sim 0.2 \mathrm{eV}$ band gap insulator with a distorted perovskite structure ${ }^{148}$. The order parameter in this parent state is charge disproportionation, with the bismuth ions nominally alternating between the $3+$ and $5+$ valence (in reality the actual charge valence disproporationation is much smaller ${ }^{53}$ ). Doping the barium site with potassium suppresses the structural distortions along with the charge disproportionation and gives rise to superconductivity with a transition temperature of nearly $30 \mathrm{~K}$ at the optimal doping.

Conventional electronic structure descriptions based on LDA/GGA fail to describe the insulating character of the parent compound. More importantly, the DFT estimates of the electron phonon coupling $\lambda$ within MigdalEliasberg theory give a value of 0.34 in the doped compound, too small to account for its superconductivity ${ }^{150}$. Careful examination found that $\lambda$ is substantially enhanced relative to its DFT estimate to a value of nearly 1.0 , and that this enhancement is responsible for superconductivity in doped $\mathrm{BaBiO}_{3}{ }^{53}$.

It was proposed that static correlations similarly enhance the electron phonon coupling in other materials proximate to an insulating state, accounting for superconductivity in systems such as $\mathrm{HfNCl}$, borocarbides and buckminsterfullerenes. For these materials, the most important type of correlation that must be captured is the static contribution, and a GW or hybrid DFT calculation is therefore necessary to correct the electronic structure. After these calculations are done, one is left with a strongly-coupled electron-phonon system with $\lambda \sim 1$. This coupling induces a large dynamical self energy, which accounts for the observed anomalous optical properties of doped $\mathrm{BaBiO}_{3}{ }^{151}$ at energies below $1 \mathrm{eV}$. Hence the low energy electron phonon treatment requires DMFT.

Setting the targets and Framing the questions. Heuristic considerations - Having identified a superconducting mechanism, namely (static) correlation enhanced electron phonon coupling it is natural to seek a realization of this mechanism in a new material. This task was undertaken by Z. Yin et al. in Ref. 153 . The parent compound would need to exhibit charge disproportionation and the value of the electron-phonon coupling must be underestimated by LDA/GGA. The heuristic reasoning used to select $\mathrm{CsTlCl}_{3}$ follows by analogy with $\mathrm{BaBiO}_{3}$. It requires an ion which would charge disproportionate. Like bismuth, thallium is known to valence skip, preferring either a $1+$ or $3+$ valence state. We want also the same perovskite structure which requires the $A \mathrm{Tl} X_{3}$ composition. Balancing the ionic charges in the presence of the average $2+$ charge of the thallium adds the next constraint. Taking ionic radii into consideration, one arrives at $\mathrm{Cs}$ for the $A$ site, and $\mathrm{Cl}$ (or $\mathrm{F}$ ) for the anion $X$ and lead to the proposal of $\mathrm{CsTlCl}_{3}$ as a valence disproportionation compound, which could be turned (by application of pressure and doping) into another "other"

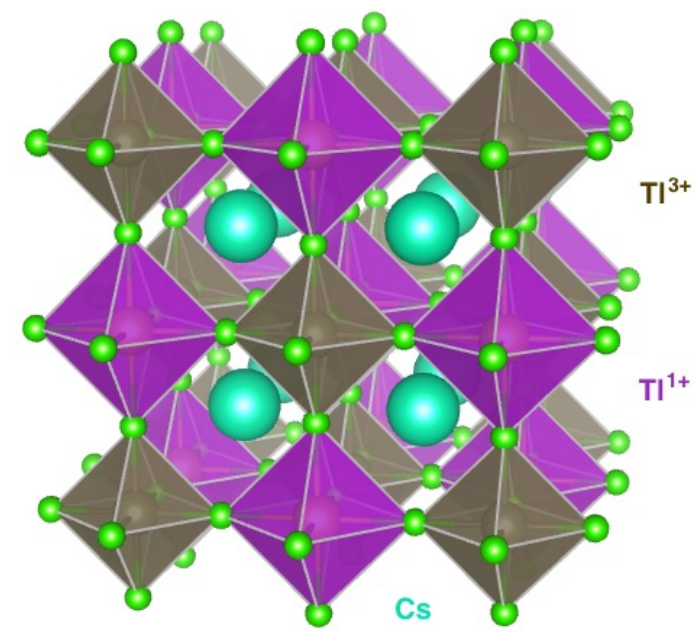

FIG. 28. Observed perovskite structure of cubic phase of $\mathrm{CsTlCl}_{3}$. The thallium ion disproportionates in to $\mathrm{Tl}^{1+}$ and $\mathrm{Tl}^{3+}$ nominally, and the structure displays an associated alternating expansion and contraction of the $\mathrm{TlCl}_{6}$ octahedral cages. Adapted from Ref. 152.

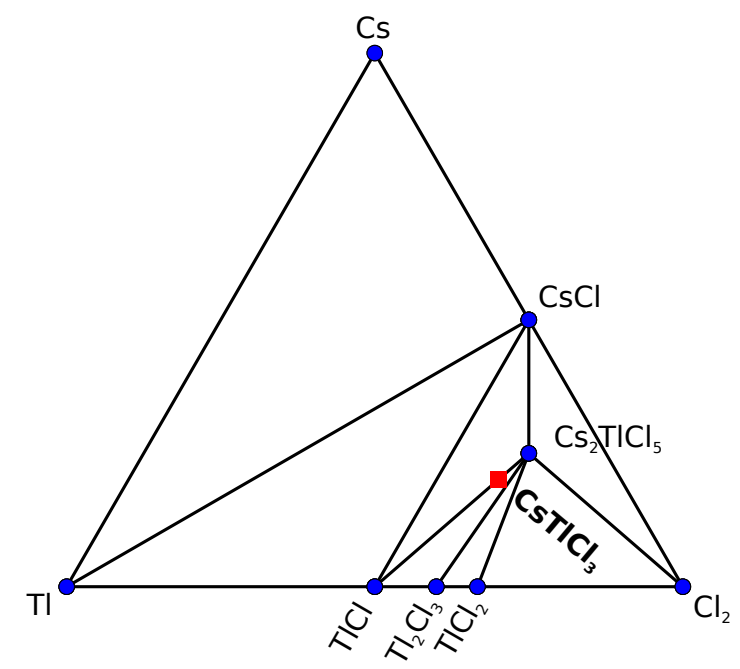

FIG. 29. Gibbs phase diagram of the Cs-Tl-Cl chemical system. The target compound $\mathrm{CsTlCl}_{3}$ is found to lie $3 \mathrm{meV} /$ atom above the convex hull. Although energies above the hull strictly imply instability, its small value is indistinguishable from zero given the systematic uncertainties in current DFT total energies (up to $50 \mathrm{meV} /$ atom), and many compounds are known to be metastable, lying up to $100 \mathrm{meV} /$ atom above the hull. The $3 \mathrm{meV} /$ atom result should be interpreted as a green light to proceed with further investigation as the structure is not obviously unstable.

high temperature superconductor ${ }^{153}$.

Electronic structure - Identification of the magnitude and nature of the correlations are an important first step for a successful electronic structure prediction, which will link the structure of the material to the desired property. In order to compute the electronic structure, it was assumed that the structure of $\mathrm{CsTlCl}_{3}$ would be the desired perovskite crystal structure, shown in Fig. 28. 

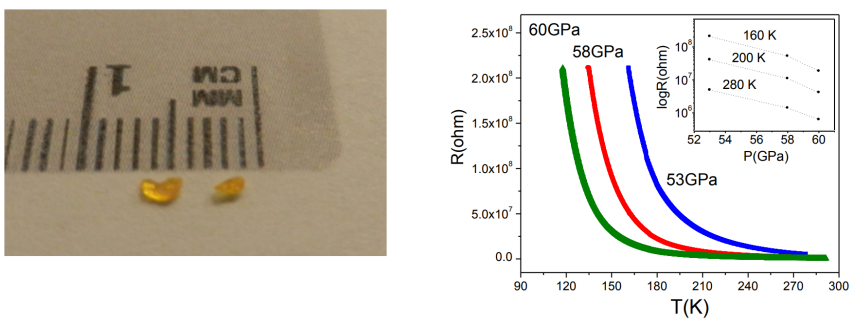

FIG. 30. $\mathrm{CsTlCl}_{3}$ single crystals resulting from successful material design (left). This material exhibits enhanced electronphono coupling and is close to metalization with pressure (right), as predicted theoretically (from Ref. 152)

To establish that this material is another potential "other high temperature superconductor" the electron-phonon coupling was evaluated using both LDA and methods that capture static correlations, namely screened hybrid density functional theory using the HSE06 functional and GW ${ }^{53}$. The calculated electronphonon couplings at $2.2 \mathrm{GPa}$ with 0.35 hole doping/f.u. (using the virtual crystal approximation) are 0.91 and 2.32 for LDA and HSE06 functionals, respectively ${ }^{153}$.

The GW method was used as a benchmark to determine the value of the HSE06 screening parameter that best reproduced the GW band structure. It was also veritfied using this methodology that takes into account static correlations that charge disproportionation occurs at half filling ${ }^{53}$.

The phase diagram of the material was then determined as a function of pressure and doping. It did resemble the phase diagram of Fig. 27. Furthermore, the electron-phonon coupling strength $\lambda$ attains a value of 2.32 at $2.2 \mathrm{GPa}$, and the predicted superconducting transition temperature is $21 \mathrm{~K}$. These results encouraged the pursuit of experimental synthesis.

Structural prediction - Structural stability was tested by computing the most important phonon modes within DFT and verifying that none were imaginary. It would be interesting to investigate whether this structure would be correctly predicted by the methods described in section VIE, and what additional polymorphs are possible in this new class of materials.

Global stability - In the original work of Ref. 153, only a few reaction pathways against known binaries were checked and the authors reported finding exothermic reactions. We now check the stability of $\mathrm{CsTlCl}_{3}$ for decomposition against all known elements, binaries and ternaries in the $\mathrm{Cs}-\mathrm{Tl}-\mathrm{Cl}$ chemical system. Using the computed total energies stored in the Materials Project database $^{3}$ and the phase diagram ${ }^{141,154}$ functionality provided with pymatgen ${ }^{155}$, we construct the convex hull plotted in Fig. 29. We find that $\mathrm{CsTlCl}_{3}$ lies $3 \mathrm{meV} /$ atom above the convex hull, indicating that the material is very likely to form.

Lessons learned - Orange crystals of $\mathrm{CsTlCl}_{3}$ were successfully grown, which indeed adopt a perovskite structure $^{152}$ exhibiting strong breathing distortions indicative

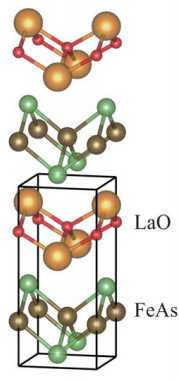

$\mathrm{LaFeAsO}$

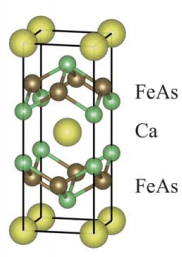

$\mathrm{CaFe}_{2} \mathrm{As}_{2}$

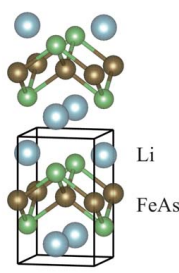

$\mathrm{LiFeAs}$

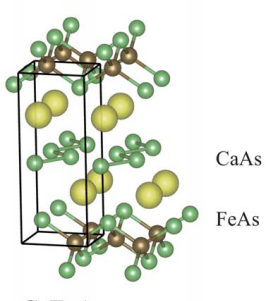

$\mathrm{CaFeAs}_{2}$
FIG. 31. Crystal structures of representative Fe based superconductors. They have common FeAs layers but different spacers.

of charge disproportionation at the thallium sites as predicted by theory. The optical gap determined by absortion measurments was of the order of $2.1 \mathrm{eV}$, in close agreement with the GW theoretical predictions ${ }^{152}$. The crystals are shown in Fig. 30

Pressure studies showed that as predicted, the compound was very close to metallization. So clearly in this case, theoretical considerations predicted an interesting material which was not listed in the ICSD database, and established that the electron phonon coupling is enhanced over its LDA value due to static correlations. These halide perovskiets are therefore a new arena to explore strong electron phonon coupling.

This compound turned out to be challenging to dope: doping via $\mathrm{Cs}$ vacancies, replacement of $\mathrm{Cl}$ by $\mathrm{O}, \mathrm{S}$, or $\mathrm{N}$, and substitution of $\mathrm{Tl}$ by $\mathrm{Hg}$ all did not succeed in pushing the material into a metallic state ${ }^{156}$. This deserves more careful investigation to see if it is the result of phonon induced self-localization or disorder. The dopablity of a material, and identification of the most probably dopants was intensely studied in weakly correlated semiconducting materials ${ }^{157}$ and is an outstanding open problem for correlated insulators and semiconductors worth pursuing.

Regardless of whether superconductivity will eventually be discovered in these thallium halides, we found that when motivated by a guiding principle, materials design of a correlated material is possible and yields not only new materials but valuable insights into the challenges (some unforseen) required to be overcome for successful end-to-end materials design. Furthermore, another polymorph phase was also found in the experiment, but since an exhaustive structural search had not been performed, it is not possible to ascertain if this phase is among the low lying structures.

\section{Fe 112 compounds}

As a final example of the use of material design to elucidate the mechanism in correlated superconducting materials, we turn our attention into iron-based superconductors. The discovery of the high temperature super- 
(a)
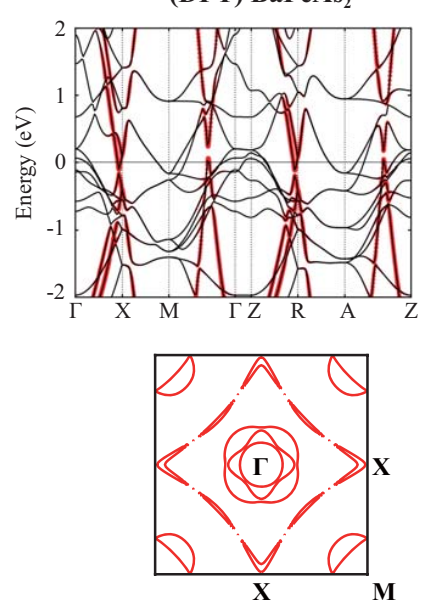

(b)
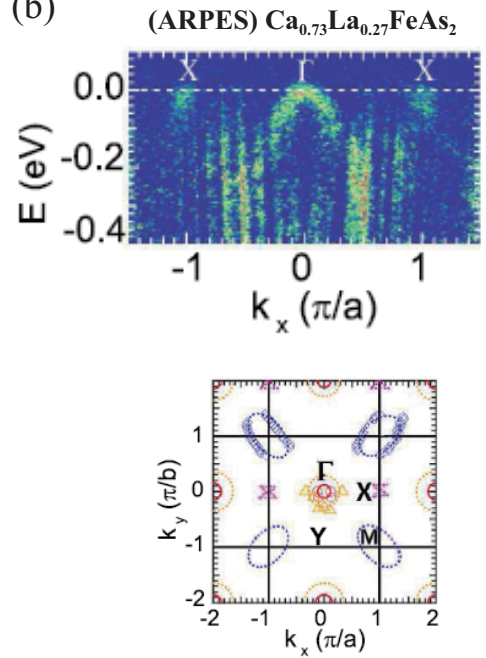

(c)
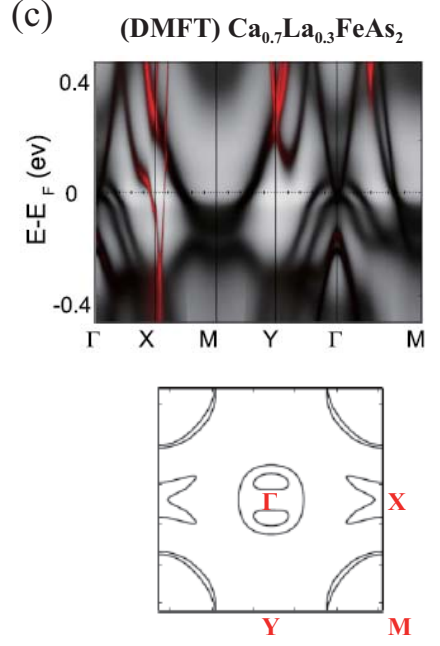

FIG. 32. Electronic structures of Fe 112 compounds. (a) Band structure (top) and Fermi surface (bottom) of the hypothetical $\mathrm{BaFeAs}_{2}$ material, computed by DFT. (b) Angle-resolved photoemission spectroscopy (ARPES) $k-E$ map (top) and the two-dimensional (2D) contour of ARPES Fermi surface (bottom) of $\mathrm{Ca}_{0.73} \mathrm{La}_{0.27} \mathrm{FeAs}_{2}$. (c) Spectral function $A(k, \omega)($ top) and Fermi surface (bottom) of $\mathrm{Ca}_{0.7} \mathrm{La}_{0.3} \mathrm{FeAs}_{2}$ computed by DMFT. The in-plane $p$ orbital characters $\left(p_{x}\right.$ and $\left.p_{y}\right)$ of the metallic As spacer are highlighted in red in the band dispersions of (a) and (c). The 2D Fermi surfaces in (a), (b), and (c) are obtained at $k_{z} \sim \pi / c$. (b) and (c) are adapted from Ref. 158 .

conducting iron-based materials by Hosono et al. ${ }^{159}$ triggered a heated debate about the superconducting mechanism in these materials. The original high $T_{c}$ superconductor was in the 1111 structure, followed by the 111 and the 122 structure (see Fig. 31). In all these cases the spacer layer is inert ( $\mathrm{LaO}$ for the $1111, \mathrm{Sr}++$ or $\mathrm{Ca}++$ for the 122 case, $\mathrm{Li}+$ in the 111 case) in the sense that their electrons are far from the Fermi level.

Following the discovery of these materials, multiple ideas where put forward in order to understand the origin of their superconductivity. One set of ideas stresses the importance of the magnetism of the iron ions, as in spin fluctuation theories ${ }^{160-163}$.

A different school of thought posits that what is important is the high electronic polarizability of the pnictides and chalcogenides. In the latter case one could envision that presence of additional metallic spacer layers would modify the polarizability and will strongly modify the superconducting transition temperature as in the polaronic mechanism of Ref. 164. This may also be the case if the superconductivity is mediated by orbital fluctuations 165. Designing an iron pnictide superconductor, with a metallic spacer layer and studying how it affects the critical temperature would help elucidate the mechanism of superconductivity in this important class of compounds. This task was undertaken by Shim et al. ${ }^{166}$.

Electronic structure - A new family of iron based superconductors, the 112 family was proposed theoretically based on chemical analogies and density functional studies ${ }^{166}$. Analogies with known 1111 compounds and the spin fluctuation mechanism lead the authors of Ref. 166 to suggest that $\mathrm{BaFeSb}_{2}$ and $\mathrm{BaFeAs}_{2}$ would be high temperature superconductors, but unlike the known structures at the time the 112 structure would have ac- tive spacer layers, where electrons living in the spacer layers contribute their own Fermi surface as shown in Fig. 32(a).

Attempts to synthesize iron pnictide materials in the 112 structure proposed in Ref. 166 were not originally successful, but new Mn-based materials in this structure were found ${ }^{167,168}$ and both theories ${ }^{167,169}$ and experiments ${ }^{168,170-172}$ show that the spacer layers in the Mn-based materials possess Dirac cones. New Ag-based materials in this structure were also reported to possess Dirac cones ${ }^{173-175}$.

Structure prediction - The original calculations of Ref. 166 relaxed lattice parameters but did not examine the local stability of the CaAs spacer layer, and had not considered the stability against phase separation. Motivated by the earlier theoretical work and experimental developments ${ }^{176,177}$ these issues were reexamined in Ref. 178, which investigated the crystal structure of $\mathrm{CaFeAs}_{2}$ by using USPEX in conjunction with VASP as DFT engine. A GGA(PBE) functional and a dense Monkhorst-Pack sampling grid with a resolution of $2 \pi \times 0.02 \AA^{-1}$ for the $k$-space integrations. In the structural search in USPEX, two formula-units per unit-cell were considered. The evolutionary structure prediction preformed by USPEX predicts that $\mathrm{CaFeAs}_{2}$ has monoclinic $P 2_{1} / m$ structure with the distortion of the CaAs layer into zigzag chains.

Global stability - A study of the thermodynamic phase stability of $\mathrm{CaFeAs}_{2}$ was performed in Ref. 178. It is reproduced in Fig. 33. Taking into account stripe magnetic order in the predicted monoclinic $P 2_{1} / m$ structure of $\mathrm{CaFeAs}_{2}$ because the additional symmetry breaking due to magnetic order (for example, antiferromag- 


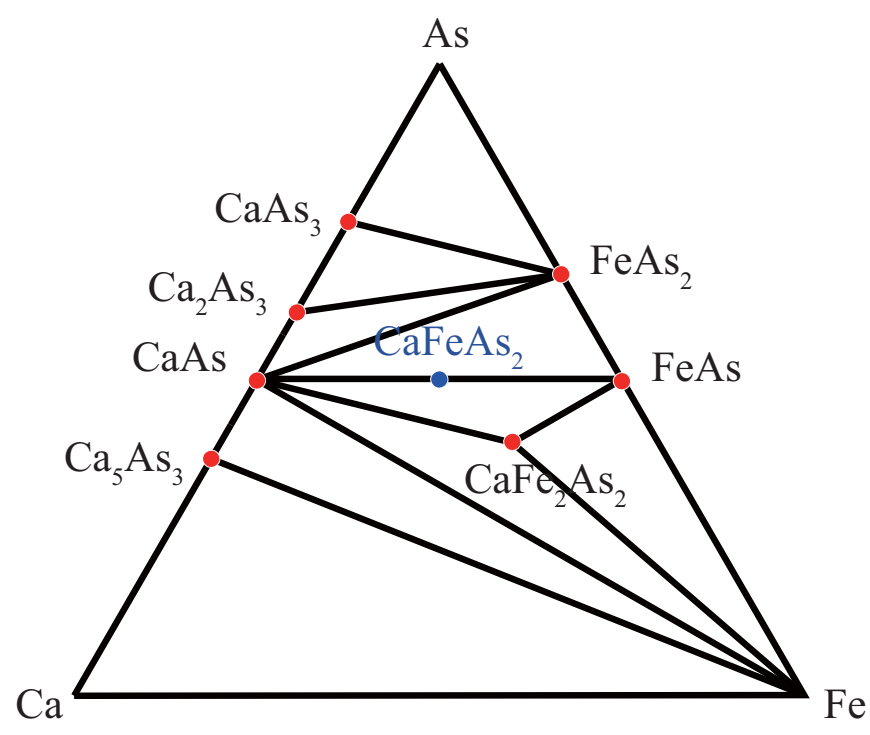

FIG. 33. Ternary phase diagram for $\mathrm{CaFeAs}_{2}$. $\mathrm{CaFeAs}_{2}$ is put above the convex hull and the energy above hull is $13 \mathrm{meV} /$ atom. Adapted from Ref. 178.

netic order) is not considered in the structural prediction performed by USPEX. Due to the stripe magnetic order, the monoclinic $P 2_{1} / m$ structure is further relaxed into a triclinic $P \overline{1}$ structure and the total energy is lower by $19.50 \mathrm{meV} /$ atom (the magnetic moment is $\left.1.95 \mu_{B} / \mathrm{Fe}\right) . \mathrm{CaFeAs}_{2}$ lies $13 \mathrm{meV} /$ atom above the convex hull, which corresponds to the existence probability of 0.37 as discussed in section V. Doping it with rareearth ions could help its stability with possible energetic gain or an entropic gain regarding no ordering of the rare-earth atoms ${ }^{178}$. Similar hypothetical compounds $\mathrm{SrFeAs}_{2}$ and $\mathrm{BaFeAs}_{2}$ were found to be 24 (existence probability: 0.33 ) and $17 \mathrm{meV} /$ atom (existence probability: 0.36 ) above the convex hull, respectively ${ }^{178}$, which are slightly higher in energy compared to $\mathrm{CaFeAs}_{2}$.

Revising the phase stability calculation - using a more accurate correlated method, as suggested in section V, we consider the determinant reaction. This reaction is found by GGA to be $\mathrm{CaAs}+\mathrm{FeAs} \rightarrow \mathrm{CaFeAs}_{2}$. The onsite Coulomb interaction $U=5 \mathrm{eV}$ and Hund's coupling constant $J=0.8 \mathrm{eV}$ were used in the Gutzwiller calculations ${ }^{158,178}$. We found that $\mathrm{CaFeAs}_{2}$ lies $7 \mathrm{meV} /$ atom above the hull.

Electronic structure - The existence of an extra Fermi surface with CaAs character in $\mathrm{Ca}_{1-x} \mathrm{La}_{x} \mathrm{FeAs}_{2}$ was confirmed by angle-resolved photoemission and the photoemission spectra is in good agreement with DFT+DMFT calculation $^{158}$ (see Figs. 32(b) and (c)). Since the CaAs layer possesses the conducting zig-zag As chain, it induces the large electronic anisotropy ${ }^{178}$.

Conclusion - Recently, iron-based superconductors in the 112 structure were synthesized with rare-earth doping, $\mathrm{Ca}_{1-x} \mathrm{La}_{x} \mathrm{FeAs}_{2}{ }^{176}$ and $(\mathrm{Ca}, \mathrm{Pr}) \mathrm{FeAs}_{2}{ }^{177}$. Surprisingly, these materials form in a structure where the As in the CaAs layers are distorted in zigzag chains (see

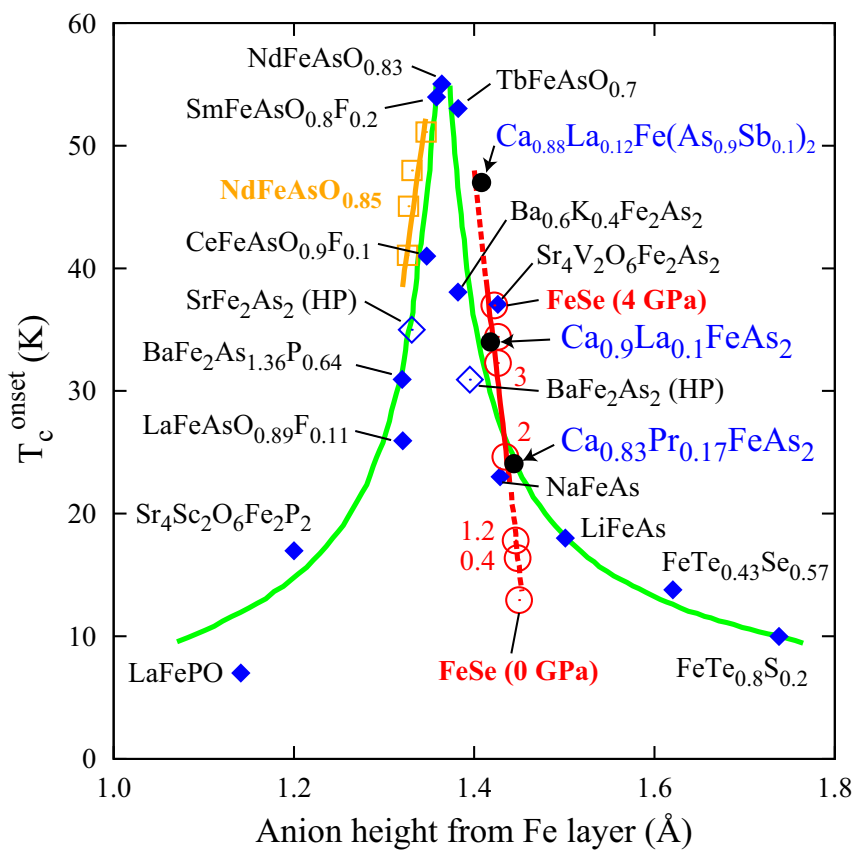

FIG. 34. $T_{c}$ vs anion height in iron pnictide and chalcogenide superconductors. Adapted from Ref. 178.

Fig. 31). The space group is monoclinic either $P 2_{1}{ }^{176}$ or $P 2_{1} / m^{177}$ rather than the originally assumed tetragonal structure. Second harmonic generation experiments confirmed the space group $P 2_{1}$ for La-doped compounds ${ }^{179}$, but similar data are absent for other rare-earth doping compounds to the best of our knowledge.

Armed with this information, the critical temperature of the newly discovered 112 compounds was replotted on the graph of Mizuguchi et al. ${ }^{180}$ which displays the $T_{c}$ as a function of the pnictogen height (see Fig. 34). The points do not deviate much from their universal plot, indicating that the fate of the superconductivity resides primarily in the FeAs layers, and is not affected by the nature of the spacer layers. This observation helps rule out a charge fluctuation mechanism in favor of spin mediated superconductivity in the iron pnictides.

More recently theoretical studies focused on the spacer layers found that the As $p_{x}$ and $p_{y}$ orbitals are responsible for the Dirac cones, and that spin-orbit coupling could not only open a gap, but also induce topological phases on these layers. This suggests that the 112 compounds are prime candidates for proximity induced topological superconductivity $^{181,182}$. More generally the 112 structure provides inspiration for combining iron pnicitide layers with layers having non-trivial topological band structure. This area of research is as yet unexplored, and calls for a new iteration of the material design loop outlined in Fig. 9. 


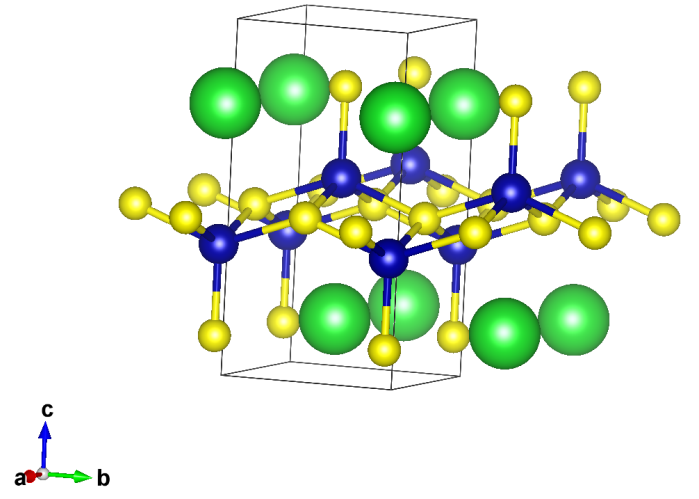

FIG. 35. Crystal Structure of the 2-dimensional parent material, $\mathrm{BaCoS}_{2}$. The green, blue, and yellow spheres correspond to $\mathrm{Ba}, \mathrm{Co}$, and $\mathrm{S}$ atoms, respectively.

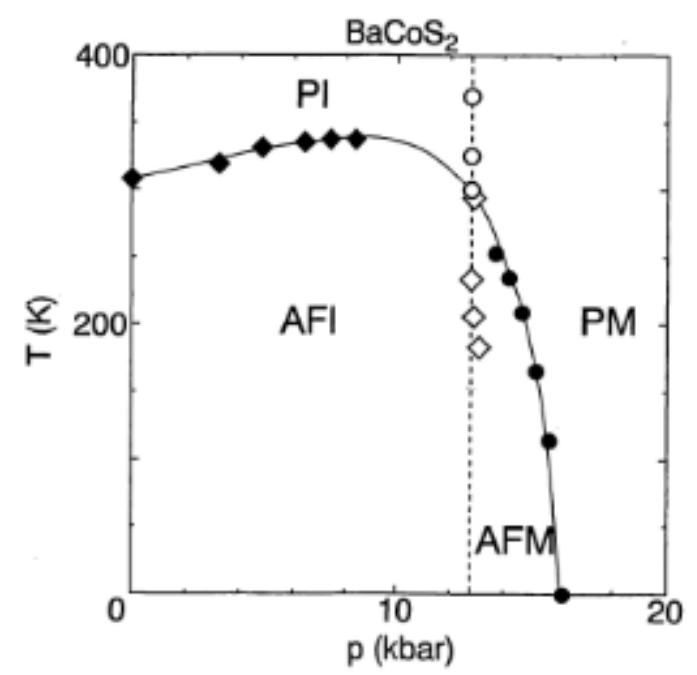

FIG. 36. $\mathrm{BaCoS}_{2}$ phase Diagram from Ref. 183, (c) (1999) The Physical Society of Japan.

\section{E. BaCoSO}

Motivation - Finally we return to the organizing principle, shown in Fig. 27, that exotic phases are often found upon suppression of a parent ordered phase of layered quasi-two-dimensional compounds. The material $\mathrm{BaCoS}_{2}$ (Fig. 35) is a layered antiferromagnetic Mott insulator. The application of pressure does suppress the magnetic order, but rather than finding a new phase at the critical point, the material simply becomes metallic down to the lowest temperatures measured as shown in Fig. 36.

Clearly, the expectation of novel phases near the suppression of order is not a sure bet! Other factors must be involved. Still this material is quite interesting, as it dis-

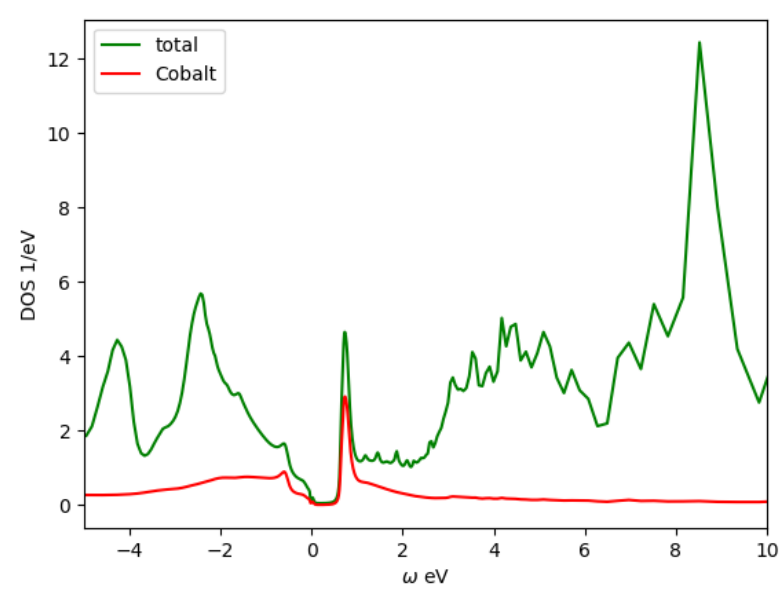

FIG. 37. Spectral function for $\mathrm{BaCoS}_{2}$ at $\mathrm{T}=180 \mathrm{~K}$ using LDA+DMFT code $^{48,49}$.

plays a transition or crossover from a paramagnetic insulating phase to a metallic phase at high temperatures and antiferromagnetically ordered state at low temperatures, features reminiscent of $\mathrm{V}_{2} \mathrm{O}_{3}$ and $\mathrm{NiSe}_{x} \mathrm{~S}_{1-x}$, hence a comparative study of these systems can illuminate the factors that govern the correlation induced metal to insulator transition.

Targets and questions - The question we address is how the physical properties of $\mathrm{BaCoS}_{2}$ change if we substitute the large sulfur ion by the smaller oxygen ion in $\mathrm{BaCoS}_{2}$ to form $\mathrm{BaCoSO}$, in particular how does the gap change as a result. At first sight one would expect that the larger size of the sulfur ion would result in a larger gap, but the answer to this question is not obvious since structural changes can take place. We approached this problem as a case study of our methodology before this material was reported in the experimental literature ${ }^{184}$. Some of the results were very surprising and are reported in this section.

Electronic Structure - Previous LDA $+\mathrm{U}$ and DMFT studies of $\mathrm{BaCoS}_{2}$ were reported in Refs. 185 and 186 . We returned to this problem using LDA+DMFT using the implementation of K. Haule ${ }^{48,49}$ and display the results in Fig. 37. Using the experimental lattice parameters, $\mathrm{BaCoS}_{2}$ is a small gap Mott insulator very close to the metal insulator transition.

Structure prediction - We continue the material design workflow by turning to structural properties of the Ba-Co-S-O system by first using USPEX, a structure prediction package based on a genetic-search algorithm, to sample the local minima in the energy landscape. We choose a 1:1:1:1 ratio for the elements and allowed for two formula units in a unit cell. We use VASP as our DFT engine. We use spin-polarized PBEsol and do not include $U$ corrections. In order not to miss crucial seed structures, which ultimately led to the experimental structure, we found that the randomly generated initial population of structures must be sufficiently large. An initial popula- 


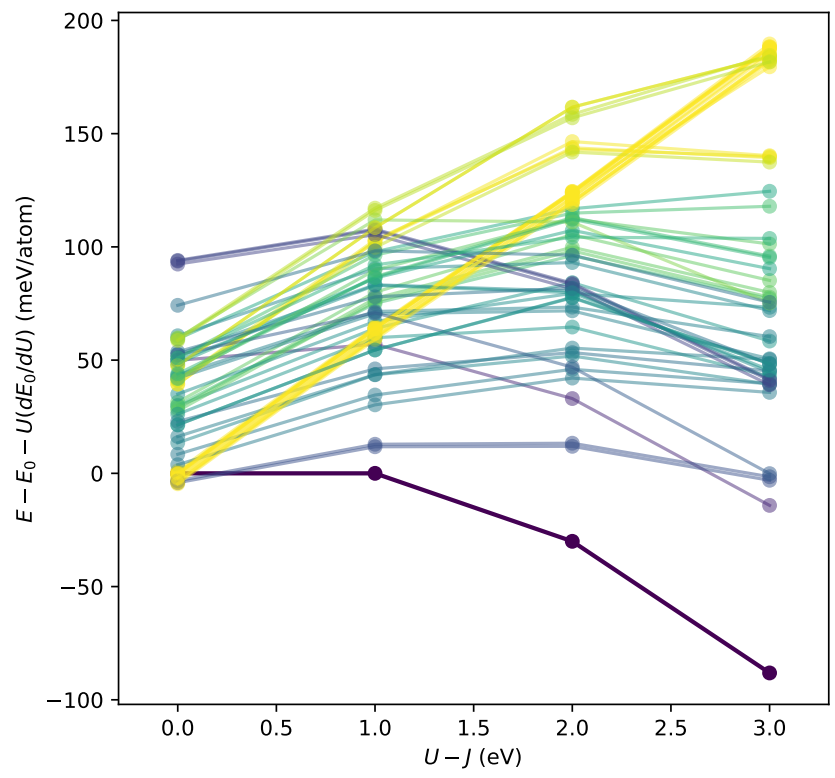

FIG. 38. The $U$-dependence of the total energies for 58 of the lowest energy structures produced by USPEX during structure prediction for BaCOSO. The energies are produced by GGA $+U$ allowing for spin polarization. The experimentally observed structure (lowest bold purple line) does not initially begin with the lowest energy at $U=0$, but rapidly becomes favored energetically with increasing correlation strength. The group of structures with the lowest energies at $U=0$ (yellow lines) rapidly rise nearly linearly with $U$. Lines colors are graded according to initial slopes at $U=0$. The intercept $E_{0}=-5.92 \mathrm{eV}$ and slope $d E_{0} / d U=0.19$ of the energy curve for the experimental structure have been subtracted for all curves to improve clarity.

tion of size 300 was sufficient with a single generation size of 60 . In total $\sim 700$ metastable structures were produced in 8 generations.

With GGA the observed structure does not have the lowest energy: it is $271 \mathrm{meV}$ above the lowest-energy structure, and there are 23 structures with lower energy. With spin-polarized GGA the situation is improved, but the structure is still not among the 10 lowest energy results. A naive conclusion would be that it is not the ground state. One would like to distinguish the observed structure within the low-lying set, for example within $0.5 \mathrm{eV} /$ (unit cell) of the final lowest energy structure. However, there are 58 such reported structures (out of the final set of 152 best-fit structure), even after removing similar structures.

We notice however that adding correlations to the Cobalt atom (in the form of a non-zero $U$ ) resolves this problem. The energies of the 58 lower-lying structures are examined as a function of $U(J=0$, which we plot in Fig. 38. Crystal structures are kept fixed and only electronic convergence is performed as $U$ is tuned. The lines are colored by their slope at $U=0$ which helps visual identification of the different types of curves.

We find that the inclusion of even a very small $U \sim$
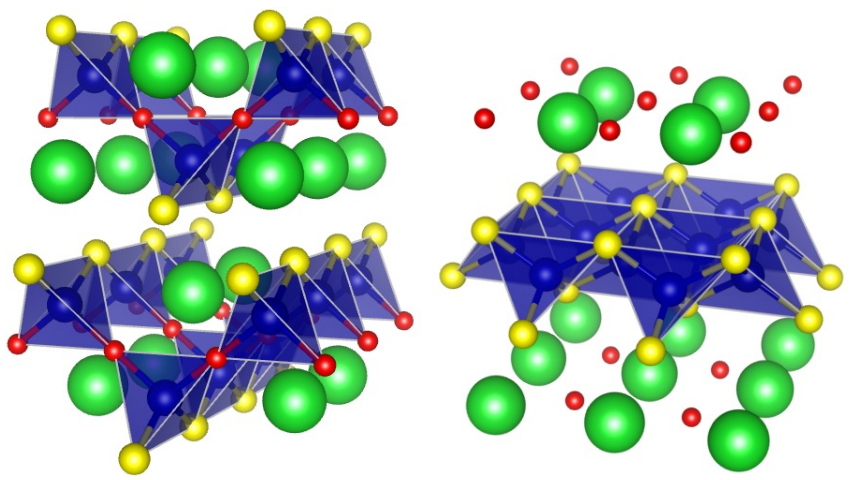

FIG. 39. Experimental structure (left), and representative of the group of lowest energy structures found by GGA (right) for the BaCoSO composition. The experimental structure contains corner-shared $\mathrm{CoS}_{2} \mathrm{O}_{2}$ tetrahedra slightly separated by $\mathrm{Ba}$ spacer layers. The GGA structure contains edgesharing $\mathrm{CoS}_{4}$ tetrahedra in a fluorite layer, which are separated by $\mathrm{BaO}$ rock salt layers.

$0.5 \mathrm{eV}$ causes a clear separation of a single structure from the remaining minima. This curve is the lowest line plotted in bold purple in Fig. 38 and corresponds to the structure shown in Fig. 39 (left). The structure contains corner-linked $\mathrm{CoS}_{2} \mathrm{O}_{2}$ tetrahedra forming a corrugated $2 D$ layer, and has been confirmed to be the correct "ground state" structure in experiment. The energy gap between the next-best structure and the ground state widens significantly as $U$ increases. The group of structures the lowest energies initially (set of diagonal yellow lines in Fig. 38) is found to be penalized most rapidly by correlations, rising nearly linearly. They correspond to slight variations of structures containing $\mathrm{CoS}$ fluorite layers separated by $\mathrm{BaO}$ rock salt layers, and a representative is shown in Fig. 39 (right). The remaining structures exhibit intermediate behavior as a function of $U$. This grouping is most clearly visualized in a plot of the slope vs. the total energy, as shown in Fig. 40. The set of structures favored by GGA form a clear outlying cluster in the upper left (yellow), the experimental structure has the smallest slope and a competitive total energy (bold square, lower left), and the remaining structures are scattered in the upper right quadrant.

In order to understand this behavior, we examine the density matrix $n_{\sigma}^{\alpha \beta}$ of cobalt extracted from the DFT computations. The indices $\alpha$ and $\beta$ run over the $3 d$ orbitals. The Coulomb $U$ term in $\mathrm{LDA}+\mathrm{U}$ has the form $\frac{U-J}{2} \sum_{\sigma} \operatorname{tr}\left\{n_{\sigma}-n_{\sigma}^{2}\right\}$, where the trace is over the orbital indices. The closer the density matrix $n_{\sigma}$ is to idempotency, the less correlations will penalize the state energetically.

From a physical viewpoint, LDA $+\mathrm{U}$ penalizes structures in which the electrons are itinerant. The average occupancy of (spin-resolved) orbitals in itinerant systems cannot be integer: strong charge fluctuations generated by hopping terms prefer occupancies near 0.5 . The $\mathrm{LDA}+\mathrm{U}$ term penalizes large values of $n_{\sigma}-n_{\sigma}^{2}$, which is maximal when the eigenvalues of $n_{\sigma} \sim 0.5$, and thus 


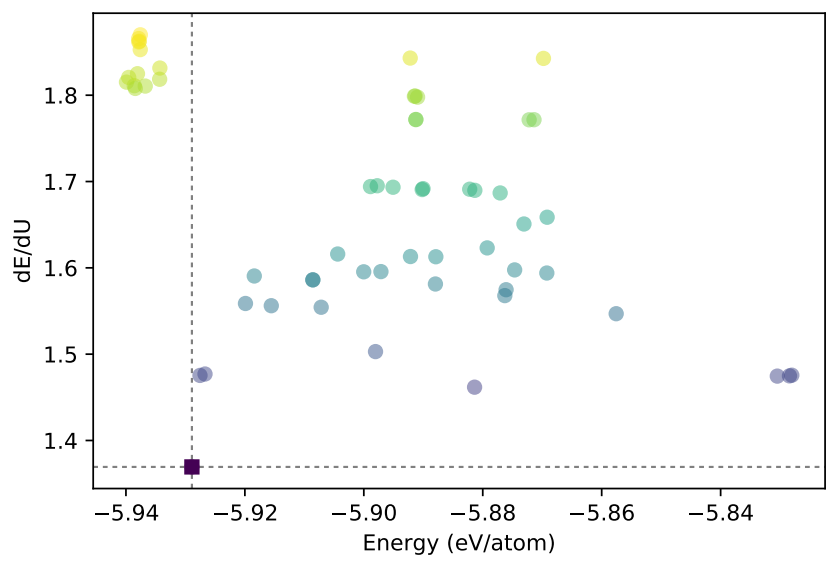

FIG. 40. Scatter plot of initial slope of the GGA+U energy curve vs. the total energy at $U=0$ for 58 candidate structures produced during the search for the crystal structure of BaCoSO. The experimental structure (lower left bold purple square) does not have the lowest energy, but has the smallest slope, so it is least penalized by increasing $U$. The group of structures with the lowest initial energies (upper left cluster of yellow dots) have large slopes, and are heavily penalized by correlations. Dotted lines centered on the experimental structure are guides to eye. Dots are colored by initial slope, matching the convention in Fig. 40.

prefers systems with nearly integer occupancies. We have plotted the initial slope of the $E(U)$ curve against this LDA $+\mathrm{U}$ term in Fig. 41. As expected, the slope and $\mathrm{LDA}+\mathrm{U}$ term track each other nearly exactly. The experimental structure has orbitals with occupancies nearest to integral, and is least penalized by correlations. The structures favored by GGA have the most itinerant orbitals, likely due to the strong covalent network of $\mathrm{CoS}_{4}$ tetrahedra in its fluorite block, and are strongly disfavored by $U$.

Our proposed strategy for structural prediction is as follows: first perform USPEX runs with spin-polarized DFT to generate the list of structures occupying local minima in the energy landscape. Then, apply an accurate and likely more computationally expensive method (such as $\mathrm{LDA}+\mathrm{U}$ or GW) to the resulting structures to reorder the total energies to determine the true ground state structure. This is more economical than running USPEX with hundreds of calls to $\mathrm{LDA}+\mathrm{U}$, producing what we estimate to be a factor of 5 speed up or more.

Finally, we verify that our conclusion about the importance of correlation is independent of the method used. Indeed, as can be seen in Tables VI and VII, the ordering between BaCoSO-expt (the observed structure) and BaCoSO-GGA (the lowest energy structure found by GGA) is the same in Wien $2 \mathrm{~K}$ as it is in VASP GGA. Calculating the correlated energy in Gutzwiller ${ }^{75}$ combined with full-potential linearized augmented plane wave Wien $2 \mathrm{k}(\mathrm{W} 2 \mathrm{~K})^{187}$ with $U=10 \mathrm{eV}$ flips the order, and makes BaCoSO-expt favorable.

Global stability - The convex hull for the Ba-Co-S-

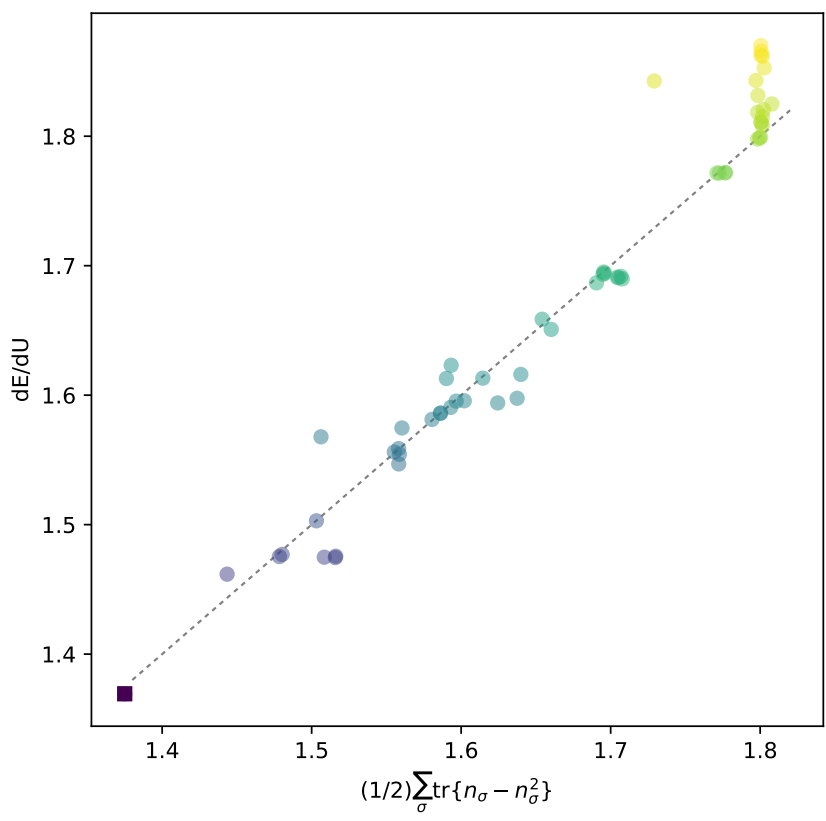

FIG. 41. Slope of energy vs. U curve plotted against the $\mathrm{LDA}+\mathrm{U}$ correlation term. Experimental structure is the bold purple square in the lower left. Dotted line is the diagonal. Dots are colored by initial slope, matching the convention in Fig. 40.

O system is shown in Fig. 42. We find BaCoSO lies $102 \mathrm{meV}$ /atom above the hull (in the corrected MaterialsProject scheme). Observing the importance of correlations in determining the correct sign or energy differences in this system, we examine the effect of correlations on the convex hull of energies.

The relevant reaction for determining the phase stability of $\mathrm{BaCoSO}$ is

$$
4 \mathrm{Co}+3 \mathrm{BaS}+\mathrm{BaSO}_{4} \rightarrow 4 \mathrm{BaCoSO}
$$

where left-hand side compounds are on the corner of the green triangle in Fig. 42.

The W2K and Gutzwiller total energies for materials in Eq. (31) are listed in Table VII. Note that we used the same on-site $U$ and Hund's coupling $J$ in Co $d$ orbitals for $\mathrm{BaCoSO}$ and Co materials to avoid the $G G A / G G A+$ $U$ correction described in Appendix A. As can be seen, GGA+U as well as ferromagnetic-Gutzwiller calculations consistently stabilize the observed material BaCoSO-expt more than BaCoSO-GGA.

Electronic Structure - Knowing the structure we can now iterate the material design loop and return to the study the electronic structure. For this purpose we use the LDA+DMFT method described in section III. We show the calculations of the spectral functions of $\mathrm{BaCoSO}$ in Fig. 43 using LDA+DMFT code ${ }^{48,49}$ and compare it to Fig. 37. The combined effects of the substitution and the structural change result in a Mott insulator with an increased gap relative to $\mathrm{BaCoS}_{2}$.

Conclusion - As mentioned earlier, BaCoSO has been 


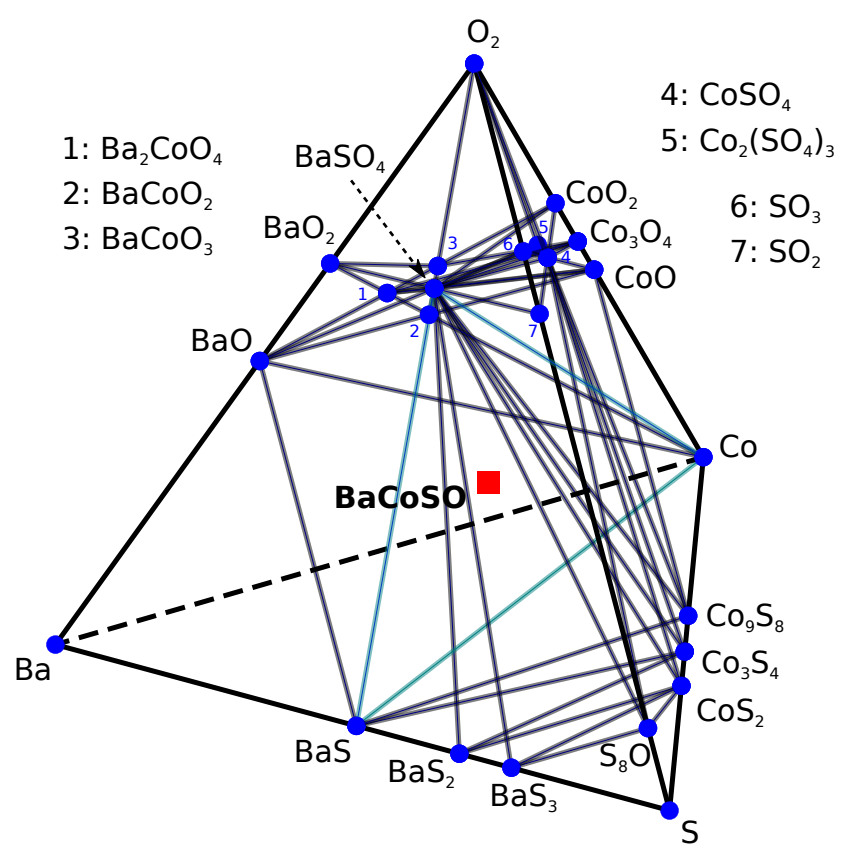

FIG. 42. Gibbs phase diagram of Ba-Co-S-O chemical system. The newly synthesized compound, BaCoSO is found to be marginally unstable, with an energy $102 \mathrm{meV} /$ atom above the hull. The compound lies on the triangular facet spanned by $\mathrm{Co}, \mathrm{BaS}$ and $\mathrm{BaSO}_{4}$.

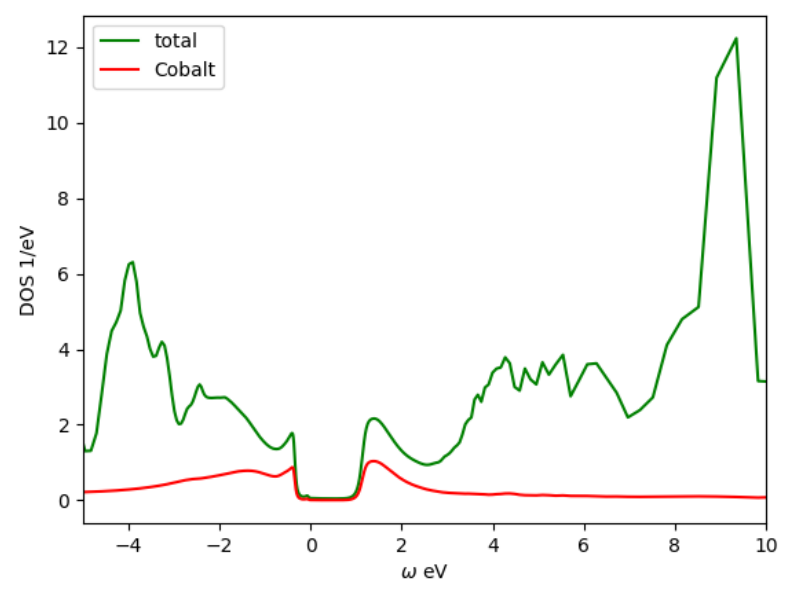

FIG. 43. Spectral function for BaCoSO at room temperature using LDA+DMFT code ${ }^{48,49}$.

very recently synthesized. It would be very useful to measure its electronic structure by photoemission and compare with the theoretical predictions. Ref. 188 theorized that it may exhibit high- $T_{c}$ superconductivity. Clearly the material design loop can continue to the next iteration.

Application of our proposed workflow raised several novel questions. How does $U$ affect the energy landscape? In particular, does $U$ simply shift the local minima relative to one another, or does it create and destroy

\begin{tabular}{l|c|cccc}
\hline & GGA & \multicolumn{4}{|c}{ GGA+U } \\
\hline Energy & bare & bare & corrected & $U$ & $J$ \\
\hline$E(\mathrm{BaS})$ & -5.106 & -5.106 & -5.438 & 0 & 0 \\
$E(\mathrm{Co})$ & -6.799 & -7.110 & -7.110 & 0 & 0 \\
$E\left(\mathrm{BaSO}_{4}\right)$ & -6.554 & -6.554 & -7.133 & 0 & 0 \\
\hline$E(\mathrm{BaCoSO}-\mathrm{expt})(\mathrm{BaCoSO}-G G A))$ & -5.853 & -5.580 & -6.390 & 3.32 & 0 \\
\hline$H^{f}(\mathrm{BaCoSO}-\mathrm{expt})$ & 0.219 & -5.570 & 0.102 & - & - \\
$\Delta H^{f}(\mathrm{BaCoSO}-\mathrm{GGA})$ & 0.163 & 0.938 & 0.471 & - & - \\
\hline
\end{tabular}

TABLE VI. Comparison of total energies $E$ and energies of formation $H^{f}$ (both in $\mathrm{eV} /$ atom) for compounds in the BaCo-S-O system via VASP pseudopotential code. The experimental and GGA-determined structures, shown in Fig. 39, are labeled BaCoSO-expt and BaCoSO-GGA. The bare values of the outputs of non-spin polarized GGA calculations are presented in the first column. For the uncorrelated calculations, the second column are the bare values of the outputs of spinpolarized GGA $(+\mathrm{U})$ calculations. These energies were modified according to the method used in the Materials Project, described in Sec. IV, to produce the corrected energies shown in the next column. The values of $U$ and $J$ (in eV) used are listed in the next two columns. In the bottom two rows, we display the formation energies, which is the result of the reaction $3 \mathrm{BaS}+4 \mathrm{Co}+\mathrm{BaSO}_{4} \rightarrow 4 \mathrm{BaCoSO}$.

minima? Additionally, when is $U$ necessary for correct reordering of the candidate energies? We expect that $U$ is only necessary for compounds containing atoms with partially-filled $d$ or $f$ shells or magnetic materials, and this hypothesis deserves to be investigated.

\section{Case Studies - wrap-up}

The examples in sections VI A-VIE showcase the question of "how" and "how well" theory can help guide discovery of new strongly correlated materials. We found that given the current limitations of existing methods, it is useful to quantify the uncertainties by thinking in statistical terms about the space of materials. This led to Fig. 14, which described the probability for the formation of a compound given its calculated energy with various methods. This should assist materials scientists in the search for new compounds, as it provides a lower bound for the probability of finding something new in a yet unexplored region of material space. As the methods for evaluation of total energies improve, this probability distribution will approach a step function centered at zero. While the evaluation of this function with the $\mathrm{LDA}+\mathrm{G}$ method is not feasible at this point, we have recalculated the energetics of the most-relevant reactions of the full LDA convex hull study, and summarized that in Table VIII.

Section VIA focused on tuning the charge transfer energy via $\mathrm{S}$ substitution to increase the superconducting critical temperature in $\mathrm{La}_{2} \mathrm{CuS}_{2} \mathrm{O}_{2}$ cuprates. The probability for this material to exist was estimated to be very 


\begin{tabular}{|c|c|c|c|c|c|}
\hline \multirow{2}{*}{ Energy } & \multirow{2}{*}{$\begin{array}{c}\text { W2K } \\
\text { PM }\end{array}$} & \multicolumn{2}{|c|}{ Gutzwiller } & \multirow{2}{*}{$U$} & \multirow{2}{*}{$J$} \\
\hline & & $\mathrm{PM}$ & FM & & \\
\hline$E(\mathrm{BaS})$ & $-116,175.236$ & $-116,175.236$ & - & 0 & 0 \\
\hline$E(\mathrm{Co})$ & $-37,917.854$ & $-37,914.456$ & $-37,914.634$ & 10.0 & 1.0 \\
\hline$E\left(\mathrm{BaSO}_{4}\right)$ & $-40,090.518$ & $-40,090.518$ & - & 0 & 0 \\
\hline$E(\mathrm{BaCoSO}-\mathrm{expt})$ & $-68,079.015$ & $-68,078.217$ & $-68,078.525$ & 10.0 & 1.0 \\
\hline$E(\mathrm{BaCoSO}-\mathrm{GGA})$ & $-68,079.077$ & $-68,078.158$ & $-68,078.169$ & 10.0 & 1.0 \\
\hline$\Delta H^{f}(\mathrm{BaCoSO}-\mathrm{expt})$ & 0.106 & 0.100 & -0.209 & - & - \\
\hline$\Delta H^{f}(\mathrm{BaCoSO}-\mathrm{GGA})$ & 0.044 & 0.159 & 0.147 & - & - \\
\hline
\end{tabular}

TABLE VII. Comparison of total energies $E$ and energies of formation $H^{f}$ (both in eV/atom) for compounds in the Ba-Co-S-O system via Wien2k and Gutzwiller calculations. The experimental and GGA-determined structures, shown in Fig. 39, are labeled BaCoSO-expt and BaCoSO-GGA. In the Gutzwiller calculations, the reference energies were paramagnetic (non-spin polarized) Wien $2 \mathrm{~K}$ calculations with $U=J=0$. Since Wien2K is an all-electron calculation, the energies are extremely large and not directly comparable to those produced using VASP in Table VI. Shown in the next two columns are the Gutzwiller total energies for paramagnetic (PM) and ferromagnetic (FM) orderings, respectively, with the Slater-Condon values of $U$ and $J$ shown in the final two columns (in $\mathrm{eV}$ ). In the bottom two rows, we display the formation energies, which is the result of the reaction $3 \mathrm{BaS}+4 \mathrm{Co}+\mathrm{BaSO}_{4} \rightarrow 4 \mathrm{BaCoSO}$. These energies are directly comparable with VASP results in Table VI. Note that the only FM Gutzwiller calculation describes the stable phase of BaCoSO-expt, suggesting that both correlation and magnetism are important to stabilize BaCoSO.

small, and indeed in-situ studies showed that it decomposes into the components predicted by the theory ${ }^{136}$. Pursuing this idea led to $\mathrm{Hg}(\mathrm{CaS})_{2} \mathrm{CuO}_{2}$ (Section VIB). It is expected to have a higher superconducting transition temperature than $\mathrm{La}_{2} \mathrm{CuO}_{4}$, but less than $\mathrm{HgBa}_{2} \mathrm{CuO}_{4}$. Its probability to exist as a ground state structure within DFT is 0.09 , but when post-processing the results with $\mathrm{LDA}+\mathrm{G}$, the material is stable, and therefore has a probability larger than 0.5 to exist.

Section VIC focused on finding new materials, where the electron-phonon coupling is enhanced by static correlations. We estimated the probability of $\mathrm{CsTlCl}_{3}$ to exist to be close to 0.5 and indeed the new materials (including the Flourine cousin) were successfully synthesized. Section VID focused on the iron pnictide, and the idea to create a new family of compounds, the 112 family, to introduce more polarizable metallic spacer layers between the active iron pnictide layers. The probability of $\mathrm{CaFeAs}_{2}$ to form was 0.37 , and indeed members of this family were synthesized.

Finally in section VIE we studied the Mott insulator BaCoSO. We showed that for this material, considerations based on LDA or GGA would have been misleading both for its ground state structure and stability against phase separation, but treating dynamical correlations gives greatly improved results. Its probability to exist according to the considerations in section $\mathrm{V}$ is 0.17 , but when the results are post-processed with $\mathrm{LDA}+\mathrm{G}$ the material becomes stable (see Table VIII) and its probability to exist is bigger than 0.5. Indeed this material was reported while this paper was in the process of being completed ${ }^{184}$, and was also successfully synthesized in the lab of M. Aronson ${ }^{189}$.

One should stress again the caveats of section $\mathrm{V}$, that the considerations leading to these probability estimates are crude, as they are based on relatively primitive estimates of energies, and ignore-finite temperature contri- butions to the entropy - both phononic and electronic. Metastable structures do form, and those are not included in ground state considerations. Estimating the probabilities of metastables states to form is problem which only now is receiving attention, see Ref. 113.

While a compound which is energetically favored will eventually form, the rate of formation is entirely controlled by kinetics of nucleation and growth. Theory of these phenomena is needed, and the 112 family illustrates this point. The formation of $\mathrm{CaFeAs}_{2}$ requires some $\mathrm{La}$ doping, even though this hardly influences the value of the total energy ${ }^{178}$.

One can use of the bounds on probability of formation discussed in this article conservatively - as a rejection criterion. There is little chance for a material to form if its probability to exist is less than 0.08 , as shown clearly in the examples of $\mathrm{La}_{2} \mathrm{CuS}_{2} \mathrm{O}_{2}$ and $\mathrm{La}_{2} \mathrm{CuSO}_{3}$ in both theory and experiment ${ }^{136}$, while all materials with probability of the order of $0.5 \mathrm{did}$ form. In intermediate cases the experimentalist needs to weigh the interest in properties of the proposed material against the probability of its formation.

Theory can of course be refined to assist further the synthesis effort. Knowing that a target compound is unstable against decomposition, one could raise the chemical potential of the products in those reactions to stabilize the desired compounds. One can also check if pressure would stabilize a desirable composition, and whether the compound would remain metastable once it is synthesized under pressure and the pressure is released ${ }^{190}$.

Overall, in the five examples discussed in this article, we see a clear consistency between the probabilistic estimates and the outcomes of the materials search process. In the process we gained a deeper understanding of how to control charge transfer energies, and how this can be used to test the mechanism of cuprate superconductivity (sections VIA, VIB). We found new compounds on 


\begin{tabular}{|c|c|c|c|c|}
\hline Material & Determinant reaction & $\Delta H_{\text {Materials proj. }}(\mathrm{eV} /$ atom $)$ & $\mathcal{P}(x)$ & $\Delta H_{\text {Gutzwiller }}(\mathrm{eV} /$ atom $)$ \\
\hline \hline $\mathrm{La}_{2} \mathrm{CuS}_{2} \mathrm{O}_{2}$ & $\mathrm{La}_{2} \mathrm{SO}_{2}+\mathrm{CuS} \rightarrow \mathrm{La}_{2} \mathrm{CuS}_{2} \mathrm{O}_{2}$ & 0.232 & 0.06 & 0.214 \\
\hline $\mathrm{La}_{2} \mathrm{CuSO}_{3}$ & $3 \mathrm{La}_{2} \mathrm{SO}_{2}+4 \mathrm{Cu}+\mathrm{La}_{2} \mathrm{SO}_{6} \rightarrow 4 \mathrm{La}_{2} \mathrm{CuSO}_{3}$ & 0.324 & 0.02 & 0.286 \\
\hline $\mathrm{Hg}(\mathrm{CaS})_{2} \mathrm{CuO}_{2}$ & $\mathrm{HgO}+2 \mathrm{CaS}+\mathrm{CuO} \rightarrow \mathrm{Hg}(\mathrm{CaS})_{2} \mathrm{CuO}_{2}$ & 0.170 & 0.09 & -0.151 \\
\hline $\mathrm{CsTlCl}_{3}$ & $\mathrm{TlCl}+\mathrm{Cs}_{2} \mathrm{TlCl}_{5} \rightarrow 2 \mathrm{CsTlCl}_{3}$ & 0.003 & 0.41 & - \\
\hline $\mathrm{CaFeAs}_{2}$ & $\mathrm{CaAs}+\mathrm{FeAs} \rightarrow \mathrm{CaFeAs}_{2}$ & 0.013 & 0.37 & 0.007 \\
\hline $\mathrm{BaCoSO}$ & $4 \mathrm{Co}+3 \mathrm{BaS}+\mathrm{BaSO}_{4} \rightarrow 4 \mathrm{BaCoSO}_{2}$ & 0.102 & 0.17 & -0.209 \\
\hline
\end{tabular}

TABLE VIII. The reaction which determines the phase stability for the materials considered in the paper (determinant reaction). Given the determinant reaction, energy above/below the hull is estimated with Materials Project corrections for DFT. Existence probability is also provided for the corresponding energy above/below the hull. Also included in the right-most column is the Gutzwiller energy above/below the hull for the given determinant reaction.

which we could study enhanced electron-phonon coupling by static correlations and its connection to valence disproportionation (section VIC), a new family of iron pnictide superconductors which can be studied to understand the mechanism of superconductivity (section VID), and a new playground to test LDA+DMFT and probe the handles that can be used to move materials around the Mott transition point (section VIE).

The examples illustrated how the different methodological aspects of the treatment of correlations were used in practice in the different stages of the material design workflow: (i) heuristic and intuitive considerations, (ii) structure to property relations, (iii) free energy evaluations and electronic structure calculations.

Structure to property. It is always useful to know whether the correlations are static or dynamic, and to which extent they are local, as this dictates the methodology used to go from the presumed structure to the properties. Section VIC treated compounds where the long range part of the Coulomb interaction is important and static correlations play a decisive role. This class of materials, which encompass $\mathrm{BaBiO}_{3}, \mathrm{HfNCl}$ and the newly designed $\mathrm{CsTlCl}_{3}$. While within LDA the electronphonon coupling constant $\lambda$ was very small, static correlations make $\lambda$ the order of unity, which results in valence disproportionation as well as high temperature superconductivity with suitable doping. These were treated using an LAPW implementation of QPGW on the Matsubara axis and hybrid DFT functionals ${ }^{27}$. Here there is a clear path for improving the treatment of materials and estimate the size of the corrections beyond QPGW. Vertex corrected treatments increase the accuracy at an increased computational $\operatorname{cost}^{27,191,192}$.

Another important direction is to simplify the $G W$ method so as to accelerate its speed - methods such as the ones proposed in Refs. 193 and 194 can be useful in this context. In Refs. 53 and 195, which led to the understanding of the electronic structure of the $\mathrm{Ba}_{1-x} \mathrm{~K}_{x} \mathrm{BiO}_{3}$ system, the HSE screened hybrid functional was used extensively with a range parameter determined by fitting the results for the more accurate QPGW. This strategy was essential in the design of the perovskite halides described in section VIC.

Sections VIE and VID illustrated the use of photoe- mission as a theoretical spectroscopy tool to examine the potential properties of a Mott insulator and a Hund's metal material. These materials are characterized by strong dynamical correlations of quite different nature, charge blocking in VIE and spin blocking in VID. Treating these classes of materials become increasingly difficult when we probe longer ranges, lower energies and lower temperatures, as unexpected emergent phenomena, such as superconductivity, appear. These more complex functionalities at low temperatures - especially predictions of superconducting transition temperatures and other broken symmetry states - are very challenging, in particular in a real materials setting. LDA + cluster DMFT on a plaquette with a zero temperature exact diagonalization solver, was the main tool used to guide the project described in sections VIA - VIB, as it is difficult to reach the very low temperature regime with Monte-Carlo methods. Work on Monte-Carlo methods to alleviate the minus sign problem and on alternative accurate finite temperature impurity solvers to cover this important region, are under active development in many groups around the world, and advances in this area will have substantial impact in searching for interesting low temperature properties of compounds.

Structural and Thermodynamical stability - Evaluation of free energies is fundamental to structure prediction and thermodynamic stability. In the projects described in this review article only the zero temperature electronic energy was estimated. Structural stability requires entropic contributions of both vibrational ${ }^{196}$ and electronic origin. For the latter, LDA+DMFT methods will be very valuable ${ }^{197,198}$. Since LDA+DMFT calculations of free energies are very time consuming, more approximate methods such as $\mathrm{LDA}+\mathrm{G}$ have been developed $^{76,199,200}$. Extension of these methods to finite temperatures is an active area of research ${ }^{201-203}$.

Strong correlations are known to affect structural energy differences. A striking example is its influence on the phase diagram of elemental Plutonium ${ }^{75}$. Other examples abound in transition metal oxides, where improvements over LDA predictions were made using hybrid functionals, LDA+U methods ${ }^{204}$, the random phase approximation (RPA $)^{205}$, and Monte-Carlo methods ${ }^{206}$, and Gutzwiller methods ${ }^{207}$. 
In section VIE we showed that correlations are important for structural predictions in a material near the Mott transition - BaCoSO. They can be incorporated first at the $\mathrm{LDA}+\mathrm{U}$ or the $\mathrm{LDA}+\mathrm{G}$ level. While incorporating these into every step of an exhaustive search such as US$\mathrm{PEX}$ is expensive, it is enough to consider them on a smaller subset, by post-processing the results of extensive LDA studies, as we demonstrated in section VIE.

For thermodynamic phase stability, the need to incorporate correlations is more widely accepted and GGA+U is used in all the Materials Projects ${ }^{3-5}$. However, this is not enough to achieve the necessary accuracy, and empirical corrections, which can be significant in magnitude, need to be added.

It is important to achieve accuracy using truly ab-initio methods. There is intensive work on using more elaborate density functionals ${ }^{208}$, or $\mathrm{RPA}^{209}$. LDA+DMFT with $\mathrm{U}$ determined from first principles and GW+DMFT are promising directions. This is important, as the values of $U$ currently used for total energy within $\mathrm{LDA}+\mathrm{U}$ are quite different from the ones needed to describe the photoemission spectra in strongly correlated materials.

We advocated recomputing total energies with more advanced methods as post-processing - after full LDA relaxations and searches. We found that this postprocessing to treat correlation, using $\mathrm{LDA}+\mathrm{U}$ and $\mathrm{LDA}+\mathrm{G}$ improves bolth structural prediction and the estimation of the probability of formation of this compound. In the future, development of forces for $\mathrm{LDA}+\mathrm{G}$, improvement in speed in the forces in LDA+DMFT and automation of user input for electronic methods, could enable a more self-consistent treatment of correlations in structural relaxation and searches.

Developing heuristics and simplified models - In addition to theory and computational tools, rapid material design requires intermediate layers of inference, lying roughly in the space currently occupied by rules of thumb such as Pauling's rules or the Hume-Rothery rules for structurally complex alloy phases. One can hope that the development of theory and computational approaches will systematize these rules as well as produce new ones.

It is important to gain some understanding of the landscape in the space of materials, and this is one of the main objectives of the materials genome initiative ${ }^{210}$. This space, as a matter of principle, is infinite as one can synthesize an infinite number of solids with molecular beam epitaxy ${ }^{211}$. Mapping a given material onto a model Hamiltonian is akin to providing some set of local coordinates relevant in this space. The hope in this exercise is that the parametrization captures important physics.

The projects described in sections VIA-VIB illustrated the usefulness of mapping onto a model Hamiltonian, parametrized by $t_{p d}, t_{p p}, U, \varepsilon_{d}-\varepsilon_{p}$ and how they can be used to explore the factors that control $T_{c}$. Just like in the case of the iron pnictides (Fig. 34), further fundamental understanding of the mechanism is needed. We have argued that designing and synthesizing new ma- terials will help in this process.

\section{CONCLUSION AND OUTLOOK}

Theory and computation have progressed to the point that they are now set to play an important role in material design and exploration, at least for weakly correlated electron materials. For strongly correlated materials, we argued that the material design process is intellectually important as it serves as a strong test of physical or chemical intuition, the quality of our methodology and predictive capabilities. We also showed through an admittedly very small number of concrete examples, that existing methodologies already have a potential to accelerate material discovery.

The materials considered in the five exemplars were relatively small variations with respect to known compounds. In this context LDA+DMFT has substantial predictive power, as one can use a fixed value of the interaction parameters, applied to a localized orbital supported on a large energy window. The LDA+DMFT double counting correction can then be determined fully from first principles as proposed in Ref. 51. Improving the accuracy of the estimates of the remaining parameters such as the strength of the screened Hubbard U and Hund's coupling J and the rest of the Slater parameters in DMFT from ab-initio would be very important for material design as we could contemplate property prediction in completely unexplored regions in the space of materials.

Combining multiple electronic structure methods to treat correlations constitutes a major software development challenge. Advances in this area will facilitate the development of quantitative measures in the space of materials, and streamline the study of materials. Such tools would enable to scale investigations, such as the ones presented in this review, from five exemplars to a statistically significant sample. It is also desirable to enable integration between electronic structure tools and the growing databases of materials and their properties. More standardization of data and databases will lead to easier integration between software and databases. Efforts in this direction have been initiated ${ }^{212}$. Finally, machine learning methods will provide alternative routes for exploring structure and property relations.

In solid state physics there is a long tradition of fruitful interactions between experiment and theory which has resulted in remarkable advances in understanding condensed matter systems. Until very recently however, materials discovery was entirely driven by experiment. This situation is rapidly changing. The highest temperature superconductor, $\mathrm{HS}_{3}$ under pressure, was synthesized ${ }^{11}$ following a material specific theoretical suggestion ${ }^{11,213}$.

When theory-assisted material design becomes reality, theory will be able to play new additional roles. Theory can help guide experiment in selecting which compositions among the thousands of possibilities would have 
the highest probability of forming a new material, and thus be fast-tracked for exploration over less promising compositions. In return, experiment can provide tests of theoretical predictions of structure and properties, while new advances in experimental techniques, where the intermediate products can be monitored in real time within the reaction vessel, will shed light into how material synthesis actually takes place. This will be a golden age for experiment - theory interactions, which has potential to take the field of correlated-electron systems to a new level.

\section{ACKNOWLEDGEMENTS}

We are grateful to our theory (Zhiping Yin, Turan Birol, C. Weber and Jihoon Shim) and experimental (Martha Greenblatt, Meigan Aronson, Ni Ni, Cedomir Petrovic and Emilia Morosan) colleagues for numerous collaborations which shaped our understanding of the material design challenge. We are grateful to Karin Rabe, Kathi Stadler, Piers Coleman and Vladan Stevanovic for useful comments on the manuscript. This work was suported by the US Department of Energy, Office of Science Basic Energy Science as a part of the Computational Materials Science Program.

\section{Appendices}

\section{A. EMPIRICAL CORRECTIONS}

We present a few of the empirical correction schemes used in literature below. All three schemes that were presented corrected GGA with GGA+U for certain elements in specific configurations. There is not complete agreement between the methods on which compounds to apply $\mathrm{U}$ to, or what its value should be. Whereas FERE, building on top of Lany's ${ }^{214}$ observation, fitted the U to prefer the correct stoichiometry between oxides with different stoichiometries, Materials Project fitted the U's on binary oxide formation enthlapies, arriving at different values. Whereas FERE applies U's universally for the set of chosen elements (although it is fitted once on oxides), Materials Project and OQMD apply GGA+U only for compounds containing oxygen or flouride. The values of chosen $\mathrm{U}$ (or $U_{\text {eff }}=U-J$ in Dudarev's method ${ }^{63}$ ) in all scheme range between $3-4 \mathrm{eV}$ in all schemes (other than one value).

In the fitted elemental-phase reference energy (FERE) scheme ${ }^{14}$, experimental formation energies $\Delta H^{\exp }$ are used to determine the best "energies" of the elemental phases. These fitted elemental-phase reference energies $E^{\mathrm{FERE}}$ are constructed to minimize the systematic error in the formation energies of the training set of compounds, and can then be used to predict the formation energies of new compounds. The procedure requires the tabulation of the experimental formation energies for 252 binary compounds $\mathrm{A}_{m} \mathrm{~B}_{n}$ along with GGA+U calculations for each of the compounds to obtain the total energies $E^{\mathrm{GGA}+\mathrm{U}}$. From this data, the elemental energies $E^{\text {FERE }}$ of the 50 elements which span the set of binaries are fitted by solving the linear least-squares problem:

$$
\begin{aligned}
\Delta H^{\exp }\left(\mathrm{A}_{m} \mathrm{~B}_{n}\right) & =E^{\mathrm{GGA}+\mathrm{U}}\left(\mathrm{A}_{m} \mathrm{~B}_{n}\right) \\
& -m E^{\mathrm{FERE}}(A)-n E^{\mathrm{FERE}}(B) .
\end{aligned}
$$

As emphasized by the authors, the energies $E^{\mathrm{FERE}}$ are not meant to improve the absolute total energies for the elemental phases, but rather constructed to optimize the systematic cancellation of errors with the GGA+U total energies. A final detail is the choice of $U$ in the GGA+U calculations. As detailed in Ref. 14, three values are used: $3 \mathrm{eV}$ for most transition metals, $5 \mathrm{eV}$ for $\mathrm{Cu}$ and $\mathrm{Ag}$, and 0 for the remaining elements. The scheme performs reasonably well: the mean absolute error of the binaries is $260 \mathrm{meV} /$ atom when computed using $\mathrm{GGA}+\mathrm{U}$, and drops to $54 \mathrm{meV} /$ atom in the FERE scheme. These improvements carry over when the fitted elemental energies are used to compute the formation energies of a test set of 55 ternaries, with the mean absolute error lying at $48 \mathrm{meV}$ /atom.

In the framework of Open Quantum Materials Database (OQMD, Ref. 4), energies of 1,670 reactions were collected, together with the corresponding GGA or GGA+U results. Unlike the FERE scheme ${ }^{14}$, they applied GGA+U only to a selected set of "correlated" elements when they are in oxides $(\mathrm{V}, \mathrm{Cr}, \mathrm{Mn}, \mathrm{Fe}, \mathrm{Co}, \mathrm{Ni}$, $\mathrm{Cu}, \mathrm{Th}, \mathrm{U}, \mathrm{Np}, \mathrm{Pu}$ ). Fitting elemental chemical potential in experimental formation energies, similarly to above Eq. (32), they compare two different empiricallybased correction schemes. In the 'fit-partial' scheme, they fit elemental energies only for those elements where the DFT energy of the $T=0 \mathrm{~K}$ ground state is not an accurate reference for room-temperature and ambient pressure (STP) formation energies. These elements include room-temperature diatomic gases $(\mathrm{H}, \mathrm{N}, \mathrm{O}, \mathrm{F}$, $\mathrm{Cl}$ ), room temperature liquids $(\mathrm{Br}, \mathrm{Hg})$, molecular solids $(\mathrm{P}, \mathrm{S}, \mathrm{I})$, elements with phase transformation between 0 $\mathrm{K}$ and room temperature ( $\mathrm{Na}, \mathrm{Ti}, \mathrm{Sn})$, and the "correlated" elements listed above. Their 'fit-all' scheme on the other hand, allows all elemental chemical potentials to be fitted. Interestingly they also find that there is considerable error within the two different experimental data sets in their database. It would be interesting to understand the source of this experimental discrepancy.

In the Materials Project, an elaborate scheme of physically-motivated energy shifts are used to respectively correct the formation energy of gases, compounds containing electronegative anions and energetic contributions due to correlations at transition metal sites ${ }^{215,216}$. The corrections in this scheme are classified as follows:

1. Gas Correction - formation-energies for gaseous elements are used as fitting variables for experimental data, similarly to the other approaches. Therefore the energies for $\mathrm{N}_{2}, \mathrm{~F}_{2}, \mathrm{Cl}_{2}$ and $\mathrm{H}_{2}$ are tabulated. 
2. Anion Correction - DFT tends to overbind the $\mathrm{O}_{2}$ molecule. It requires too much energy to dissociate $\mathrm{O}_{2}$ molecule in oxidation reactions, which results in underestimating the oxidation energy. It gives a constant energy shift compared to experimental data $^{215}$. Hence, the DFT total energy for any oxide or sulfide compound is corrected by the following negative shift, which accounts for DFT overbinding of anions:

$$
\Delta E=E \cdot N
$$

where $\mathrm{E}$ is the correction energy, and $\mathrm{N}$ is the number of $\mathrm{O}$ or $\mathrm{S}$ in the composition. If both oxygen and sulfur are present in the compound, both corrections are applied. The correction energy $\mathrm{E}$ is tabulated for the ionic state of the anion: oxide $\left(\mathrm{O}^{2-}\right)$, peroxide $\left(\mathrm{O}_{2}^{2-}\right)$, superoxide $\left(\mathrm{O}_{2}^{-}\right)$, ozonide $\left(\mathrm{O}_{3}^{-}\right)$, and sulfide $\left(\mathrm{S}^{2-}\right)$.

3. Correction for correlations $-\mathrm{GGA}+\mathrm{U}$ is applied for a selected set of transition metal compounds containing oxygen or fluorine atoms (transition metal oxides or transition metal fluorides) ${ }^{107,216}$. The value of $\mathrm{U}$ was fixed empirically for the following set of elements: Co, Cr, Fe, Mn, Mo, Ni, V, W.
4. Correction for $G G A+U$ vs. GGA compatibility The DFT energy for any oxide or fluoride compound is corrected if the run is a GGA+U calculation. The correction is applied to any fluoride or oxide containing one of the following transition metals: $\{\mathrm{V}, \mathrm{Cr}, \mathrm{Mn}, \mathrm{Fe}, \mathrm{Co}, \mathrm{Ni}, \mathrm{Mo}, \mathrm{W}\}$. Note $\mathrm{Ti}$ and $\mathrm{Cu}$ are absent. The corrections are of the form:

$$
\Delta E=\sum_{i} E_{i} \cdot N_{i}
$$

where $i$ runs over the transition metals, $N_{i}$ is the number of atoms of the transition metal present in the compound, and $E_{i}$ is the correction energy is tabulated.

While the corrections are semi-empirical, they perform well from a practical standpoint, allowing formation energies to be accurately determined to within 25 $50 \mathrm{meV} /$ atom as compared to experimental thermodynamic benchmarks and successfully reproduce Gibbs phase diagrams ${ }^{14,215,216}$. In the paper we used the Materials Project software to construct convex-hulls and extract energies-above-hull ${ }^{141,216}$. Therefore the corrections that we apply are based on their scheme.
${ }^{1}$ L. H. Greene, H. Z. Arham, C. R. Hunt, and W. K. Park, J Supercond Nov Magn 25, 2121 (2012).

${ }^{2}$ K. Lejaeghere, G. Bihlmayer, T. Björkman, P. Blaha, S. Blügel, V. Blum, D. Caliste, I. E. Castelli, S. J. Clark, A. Dal Corso, S. de Gironcoli, T. Deutsch, J. K. Dewhurst, I. Di Marco, C. Draxl, M. Dułak, O. Eriksson, J. A. Flores-Livas, K. F. Garrity, L. Genovese, P. Giannozzi, M. Giantomassi, S. Goedecker, X. Gonze, O. Grånäs, E. K. U. Gross, A. Gulans, F. Gygi, D. R. Hamann, P. J. Hasnip, N. A. W. Holzwarth, D. Iuşan, D. B. Jochym, F. Jollet, D. Jones, G. Kresse, K. Koepernik, E. Küçükbenli, Y. O. Kvashnin, I. L. M. Locht, S. Lubeck, M. Marsman, N. Marzari, U. Nitzsche, L. Nordström, T. Ozaki, L. Paulatto, C. J. Pickard, W. Poelmans, M. I. J. Probert, K. Refson, M. Richter, G.-M. Rignanese, S. Saha, M. Scheffler, M. Schlipf, K. Schwarz, S. Sharma, F. Tavazza, P. Thunström, A. Tkatchenko, M. Torrent, D. Vanderbilt, M. J. van Setten, V. Van Speybroeck, J. M. Wills, J. R. Yates, G.-X. Zhang, and S. Cottenier, Science 351, aad3000 (2016).

3 A. Jain, S. P. Ong, G. Hautier, W. Chen, W. D. Richards, S. Dacek, S. Cholia, D. Gunter, D. Skinner, G. Ceder, and K. a. Persson, APL Materials 1, 011002 (2013).

${ }^{4}$ S. Kirklin, J. E. Saal, B. Meredig, A. Thompson, J. W. Doak, M. Aykol, S. Rühl, and C. Wolverton, npj Computational Materials 1, 15010 (2015).

5 W. Setyawan, R. M. Gaume, S. Lam, R. S. Feigelson, and S. Curtarolo, ACS Comb. Sci. 13, 382 (2011).

6 D. Neugebauer, J, Kormann F, Grabowski, B, Hickel T, Raabe, in Harnessing Mater. Genome Accel. Mater. Dev. via Comput. Exp. Tools, 2013 (2012).

7 C. J. Fennie, Phys. Rev. Lett. 100, 167203 (2008).
${ }^{8}$ R. Gautier, X. Zhang, L. Hu, L. Yu, Y. Lin, T. O. L. Sunde, D. Chon, K. R. Poeppelmeier, and A. Zunger, Nature Chemistry 7, 308 (2015).

9 D. J. Fredeman, P. H. Tobash, M. A. Torrez, J. D. Thompson, E. D. Bauer, F. Ronning, W. W. Tipton, S. P. Rudin, and R. G. Hennig, Phys. Rev. B 83, 224102 (2011).

10 N. P. Armitage, E. J. Mele, and A. Vishwanath, Reviews of Modern Physics 90 (2018), https://doi.org/10.1103/RevModPhys.90.015001, arXiv:1705.01111.

11 D. Duan, Y. Liu, F. Tian, D. Li, X. Huang, Z. Zhao, H. Yu, B. Liu, W. Tian, and T. Cui, Sci. Rep. 4, 6968 (2014).

12 A. P. Drozdov, M. I. Eremets, I. A. Troyan, V. Ksenofontov, and S. I. Shylin, Nature 525, 73 (2015).

13 W. Kohn and L. J. Sham, Phys. Rev. 140, A1133 (1965).

${ }^{14}$ V. Stevanović, S. Lany, X. Zhang, and A. Zunger, Phys. Rev. B 85, 115104 (2012).

15 DMFT at 25: Infinite Dimensions, Modeling and Simulation, Vol. 4, Autumn School on Correlated Electrons, Jlich (Germany), 15 Sep 2014 - 19 Sep 2014 (Forschungszentrum Jlich Zentralbibliothek, Verlag, Jlich, 2014).

16 M. S. Hybertsen and S. G. Louie, Phys. Rev. Lett. 55, 1418 (1985).

17 S. G. Louie, "First-principles theory of electron excitation energies in solids, surfaces, and defects," in Topics in Computational Materials Science, edited by C. Fong (World Scientific, Singapore, 1997) pp. 96,.

18 I. Shavitt and R. Bartlett, Many-Body Methods in Chemistry and Physics: MBPT and Coupled-Cluster Theory, Cambridge Molecular Science (Cambridge University Press, 2009). 
19 J. M. Tomczak, M. van Schilfgaarde, and G. Kotliar, Phys. Rev. Lett. 109, 237010 (2012).

${ }^{20}$ M. V. Sadovskii, I. A. Nekrasov, E. Z. Kuchinskii, T. Pruschke, and V. I. Anisimov, Phys. Rev. B - Condens. Matter Mater. Phys. 72, 1 (2005), arXiv:0508585 [condmat]

${ }^{21}$ L. Hedin, Phys. Rev. 139, A796 (1965).

22 B. Holm and U. von Barth, Phys. Rev. B 57, 2108 (1998).

23 A. Stan, N. E. Dahlen, and R. van Leeuwen, Europhys. Lett. 76, 298 (2006).

24 A. Kutepov, S. Y. Savrasov, and G. Kotliar, Phys. Rev. B 80, 041103(R) (2009).

25 S. V. Faleev, M. van Schilfgaarde, and T. Kotani, Phys. Rev. Lett. 93, 126406 (2004).

26 A. Kutepov, K. Haule, S. Y. Savrasov, and G. Kotliar, Phys. Rev. B 85, 155129 (2012).

27 A. L. Kutepov, V. S. Oudovenko, and G. Kotliar, Comput. Phys. Commun. 219, 407 (2017).

28 G. Kotliar and D. Vollhardt, Physics Today 57, 53 (2004).

29 A. Georges and G. Kotliar, Phys. Rev. B 45, 6479 (1992).

30 W. Metzner and D. Vollhardt, Phys. Rev. Lett. 62, 324 (1989).

31 E. Gull, A. J. Millis, A. I. Lichtenstein, A. N. Rubtsov, M. Troyer, and P. Werner, Rev. Mod. Phys. 83, 349 (2011).

32 V. I. Anisimov, A. I. Poteryaev, M. A. Korotin, A. O. Anokhin, and G. Kotliar, J. Phys. Condens. Matter. 9, 7359 (1997).

33 A. I. Lichtenstein and M. I. Katsnelson, Phys. Rev. B 57, 6884 (1998).

34 S. Y. Savrasov and G. Kotliar, Phys. Rev. B 69, 245101 (2004).

35 S. Y. Savrasov, G. Kotliar, and E. Abrahams, Nature 410, 793 (2001).

36 R. Chitra and G. Kotliar, Phys. Rev. B 63, 115110 (2001).

37 R. Chitra and G. Kotliar, Phys. Rev. B 62, 12715 (2000).

38 X. Dai, S. Y. Savrasov, G. Kotliar, A. Migliori, H. Ledbetter, and E. Abrahams, Science 300, 953 (2003).

39 I. Paul and G. Kotliar, Eur. Phys. J. B 51, 189 (2006).

40 J. Lee and K. Haule, Phys. Rev. B 91, 155144 (2015).

41 B. Amadon, F. Lechermann, A. Georges, F. Jollet, T. O. Wehling, and A. I. Lichtenstein, Phys. Rev. B 77, 205112 (2008).

42 B. Amadon, J. Phys. Condens. Matter. 24, 075604 (2012).

43 H. Park, A. J. Millis, and C. A. Marianetti, Phys. Rev. B 90, 235103 (2014).

44 O. Parcollet, M. Ferrero, T. Ayral, H. Hafermann, I. Krivenko, L. Messio, and P. Seth, Comput. Phys. Commun. 196, 398 (2015).

45 "Amulet: http://amulet-code.org/," .

46 "Lisa: http://dmft.rutgers.edu/lisa/,"

47 "Lmtart: http://mindlab.physics.ucdavis.edu/materialresearch /scientific/index_lmtart.htm," .

48 K. Haule, C. H. Yee, and K. Kim, Phys. Rev. B 81, 195107 (2010).

49 "Rutgers dft+edmft: http://hauleweb.rutgers.edu/tutorials/,"

50 O. Grånäs, I. D. Marco, P. Thunströma, L. Nordström, O. Eriksson, T. Björkman, and J. M. Wills, Comput. Mater. Sci. 55, 295 (2012).

${ }^{51}$ K. Haule, Phys. Rev. Lett. 115, 196403 (2015).

52 C.-O. Almbladh, U. von Barth, and R. van Leeuwen, Int. J. Mod. Phys. B 13, 535 (1999).
53 Z. P. Yin, A. Kutepov, and G. Kotliar, Phys. Rev. X 3, 021011 (2013).

${ }^{54}$ D. Jacob, K. Haule, and G. Kotliar, EPL 84, 57009 (2008).

55 S. Biermann, F. Aryasetiawan, and A. Georges, Phys. Rev. Lett. 90, 086402 (2003).

${ }^{56}$ G. Kotliar, S. Y. Savrasov, K. Haule, V. S. Oudovenko, O. Parcollet, and C. A. Marianetti, Rev. Mod. Phys. 78, 865 (2006).

57 P. Sun and G. Kotliar, Phys. Rev. B 66, 085120 (2002).

58 P. Sun and G. Kotliar, Phys. Rev. Lett. 92, 196402 (2004).

59 G. Rohringer, H. Hafermann, A. Toschi, A. A. Katanin, A. E. Antipov, M. I. Katsnelson, A. I. Lichtenstein, A. N. Rubtsov, and K. Held, Rev. Mod. Phys. 90, 025003 (2018).

60 S. Choi, A. Kutepov, K. Haule, M. van Schilfgaarde, and G. Kotliar, npj Quantum Materials 1, 16001 (2016).

61 J. M. Tomczak, M. Casula, T. Miyake, F. Aryasetiawan, and S. Biermann, EPL 100, 67001 (2012).

62 V. I. Anisimov, J. Zaanen, and O. K. Andersen, Phys. Rev. B 44, 943 (1991).

63 S. L. Dudarev, G. A. Botton, S. Y. Savrasov, C. J. Humphreys, and A. P. Sutton, Phys. Rev. B 57, 1505 (1998).

64 A. I. Liechtenstein, V. I. Anisimov, and J. Zaanen, Phys. Rev. B 52, R5467(R) (1995).

65 V. I. Anisimov, F. Aryasetiawan, and A. I. Lichtenstein, J. Phys. Condens. Matter 9, 767 (1997).

66 A. G. Petukhov, I. I. Mazin, L. Chioncei, and A. I. Lichtenstein, Phys. Rev. B 67, 153106 (2003).

67 M. T. Czyźyk and G. A. Sawatzky, Phys. Rev. B 49, 14211 (1994).

68 B. Himmetoglu, A. Floris, S. de Gironcoli, and M. Cococcioni, Int. J. Quantum Chem. 114, 14 (2014), arXiv:1309.3355.

69 G. Kotliar and A. E. Ruckenstein, Phys. Rev. Lett. 57, 1362 (1986).

70 T. Li, P. Wölfle, and P. J. Hirschfeld, Phys. Rev. B 40, 6817 (1989).

71 R. Frésard and P. Wölfle, Int. J. Mod. Phys. B 06, 685 (1992).

72 R. Frésard and G. Kotliar, Phys. Rev. B 56, 12909 (1997).

${ }^{73}$ F. Lechermann, A. Georges, G. Kotliar, and O. Parcollet, Phys. Rev. B 76, 155102 (2007).

74 R. Raimondi and C. Castellani, Phys. Rev. B 48, 11453(R) (1993).

75 N. Lanatà, Y. Yao, C.-Z. Wang, K.-M. Ho, and G. Kotliar, Phys. Rev. X 5, 011008 (2015).

76 N. Lanatà, Y. Yao, X. Deng, V. Dobrosavljević, and G. Kotliar, Phys. Rev. Lett. 118, 126401 (2017).

77 J. Bünemann and F. Gebhard, Phys. Rev. B 76, 193104 (2007).

78 X. Deng, X. Dai, and Z. Fang, EPL 83, 37008 (2008).

79 K. M. Ho, J. Schmalian, and C. Zhuang, Phys. Rev. B 77, 073101 (2008)

80 S. Y. Savrasov, V. Oudovenko, K. Haule, D. Villani, and G. Kotliar, Phys. Rev. B 71, 115117 (2005).

81 C. Piefke and F. Lechermann, Phys. Rev. B 97 (2017), https://doi.org/10.1103/PhysRevB.97.125154, arXiv:1708.03191.

82 G. Baym and L. P. Kadanoff, Phys. Rev. 124, 287 (1961).

83 G. Baym, Phys. Rev. 127, 1391 (1962).

${ }^{84}$ F. Krien, E. G. C. P. van Loon, H. Hafermann, J. Otsuki, M. I. Katsnelson, and A. I. Lichtenstein, Phys. Rev. B 
96, 075155 (2017)

85 G. Biroli, O. Parcollet, and G. Kotliar, Phys. Rev. B 69, 205108 (2004).

${ }^{86}$ D. Karlsson and R. van Leeuwen, Phys. Rev. B 94, 125124 (2016).

87 G. Stefanucci, Y. Pavlyukh, A.-M. Uimonen, and R. van Leeuwen, Phys. Rev. B 90, 115134 (2014).

88 A. Kutepov, K. Haule, S. Y. Savrasov, and G. Kotliar, Phys. Rev. B 82, 045105 (2010).

89 N. E. Zein, S. Y. Savrasov, and G. Kotliar, Phys. Rev. Lett. 96, 226403 (2006).

90 F. Nilsson, L. Boehnke, P. Werner, and F. Aryasetiawan, Phys. Rev. Materials 1, 043803 (2017).

91 T. Ayral, T.-H. Lee, and G. Kotliar, Phys. Rev. B 96, 235139 (2017).

92 S. M. Woodley and R. Catlow, Nat. Mater. 7, 937 (2008).

93 J. Pannetier, J. Bassas-Alsina, J. Rodriguez-Carvajal, and V. Caignaert, Nature 346, 343 (1990).

94 S. Kirkpatrick, C. D. G. Jr., and M. P. Vecchi, Science 220, 671 (1983).

95 S. M. Woodley, P. D. Battle, J. D. Gale, and C. R. A. Catlow, Phys. Chem. Chem. Phys. 1, 2535 (1999).

96 A. R. Oganov and C. W. Glass, J. Chem. Phys. 124, 244704 (2006).

97 G. Trimarchi and A. Zunger, Phys. Rev. B 75, 104113 (2007).

98 S. Curtarolo, D. Morgan, K. Persson, J. Rodgers, and G. Ceder, Phys. Rev. Lett. 91, 135503 (2003).

99 C. C. Fischer, K. J. Tibbetts, D. Morgan, and G. Ceder, Nat. Mater. 5, 641 (2006).

100 A. Laio and M. Parrinello, Proc. Natl. Acad. Sci. U.S.A. 99, $12562(2002)$.

101 A. Barducci, M. Bonomi, and M. Parrinello, Comput. Mol. Sci. 1, 826 (2011).

102 D. J. Wales and J. P. K. Doye, J. Chem. Phys. A 101, 5111 (1997).

103 S. Goedecker, J. Chem. Phys. 120, 9911 (2004).

104 C. J. Pickard and R. J. Needs, J. Phys. Condens. Matter. 23, 053201 (2011).

105 V. Stevanović, Phys. Rev. Lett. 116, 075503 (2016).

106 A. O. Lyakhov, A. R. Oganov, H. T. Stokes, and Q. Zhu, Computer Physics Communications 184, 1172 (2013).

107 G. Hautier, S. P. Ong, A. Jain, C. J. Moore, and G. Ceder, Phys. Rev. B 85, 155208 (2012).

108 The SQL query sent to OQMDv1.1 was: select

d.composition_id, '\#',

d.delta_e as formation_energy,

e.delta_e as dft_formation_energy,

d.source as expt_source,

e.source as dft_source

from

expt_formation_energies as d,

expt_formation_energies as e

where

d.dft $=0$ and e.dft $=1$ and

e.composition_id $=$ d.composition_id.

109 A. Watson and T. Markus, Subvolume C Ternary Steel Syst. Phase Diagrams Phase Transit. Data, Part 2 Ternary Syst. from $\mathrm{Cr}-\mathrm{Mn}-\mathrm{N}$ to $\mathrm{Ni}$-Si-Ti Ser. LandoltBörnstein Numer. Data Funct. Relationships Sci. Technol. - New Ser. Part 19C2 (2015).

110 P. Nash, "Thermodynamic database," (2013).
111 E. Jones, T. Oliphant, P. Peterson, et al., "SciPy: Open source scientific tools for Python," (2001-).

112 A. Narayan, A. Bhutani, S. Rubeck, J. N. Eckstein, D. P. Shoemaker, and L. K. Wagner, Phys. Rev. B 94, 045105 (2016).

113 W. Sun, S. T. Dacek, S. P. Ong, G. Hautier, A. Jain, W. D. Richards, A. C. Gamst, K. A. Persson, and G. Ceder, Science Advances 2, e1600225 (2016).

114 P. J. Ryan, J. W. Kim, T. Birol, P. Thompson, J. H. Lee, X. Ke, P. S. Normile, E. Karapetrova, P. Schiffer, S. D. Brown, C. J. Fennie, and D. G. Schlom, Nat. Commun. 4, 1 (2013).

115 L. Havlák, J. Fábry, M. Henriques, and M. Dušek, Acta Crystallogr. Sect. C Struct. Chem. 71, 623 (2015).

116 X. Deng, K. Haule, and G. Kotliar, Phys. Rev. Lett. 111, 176404 (2013).

117 Q. Yin, A. Kutepov, K. Haule, G. Kotliar, S. Y. Savrasov, and W. E. Pickett, Phys. Rev. B 84, 195111 (2011).

118 D. Butler, Nature 429, 238 (2004).

119 P. Söderlind, G. Kotliar, K. Haule, P. M. Oppeneer, and D. Guillaumont, MRS Bull. 35, 883 (2010).

120 Y. Yun and P. M. Oppeneer, MRS Bull. 36, 178 (2011).

121 J. Zaanen, G. A. Sawatzky, and J. W. Allen, Phys. Rev. Lett. 55, 418 (1985).

122 N. E. Bickers, D. J. Scalapino, and R. T. Scalettar, Int. J. Mod. Phys. B 01, 687 (1987).

123 G. Kotliar and J. Liu, Phys. Rev. B 38, 5142 (1988).

124 F. C. Zhang, C. Gros, T. M. Rice, and H. Shiba, Supercond. Sci. Technol. 1, 36 (1988), arXiv:0311604 [condmat].

125 M. Capone and G. Kotliar, Phys. Rev. B 74, 1 (2006), arXiv:0603227 [cond-mat].

126 C. Weber, C. Yee, K. Haule, and G. Kotliar, EPL 100 , 37001 (2012).

127 E. Pavarini, T. Saha-Dasgupta, O. Jepsen, and O. K. Andersen, Phys. Rev. Lett. 87 (2001), 10.1103/PhysRevLett.87.047003, arXiv:0406357 [cond-mat].

128 Y. Ohta, T. Tohyama, and S. Maekawa, Phys. Rev. B 43, 2968 (1991).

129 R. Raimondi, J. H. Jefferson, and L. F. Feiner, Phys. Rev. B 53, 8774 (1996).

130 H. Sakakibara, H. Usui, K. Kuroki, R. Arita, and H. Aoki, Phys. Rev. Lett. 105, 057003 (2010).

131 N. Marzari and D. Vanderbilt, Phys. Rev. B 56, 12847 (1997).

132 I. Souza, N. Marzari, and D. Vanderbilt, Phys. Rev. B 65, 035109 (2001).

133 A. A. Mostofi, J. R. Yates, Y.-S. Lee, I. Souza, D. Vanderbilt, and N. Marzari, Comput. Phys. Commun. 178, 685 (2008).

134 C. H. Yee and G. Kotliar, Phys. Rev. B - Condens. Matter Mater. Phys. 89 (2014), 10.1103/PhysRevB.89.094517, arXiv:1305.0322.

135 V. I. Anisimov, M. A. Korotin, A. S. Mylnikova, A. V. Kozhevnikov, D. M. Korotin, and J. Lorenzana, Phys. Rev. B 70, 172501 (2004).

136 H. He, C.-H. Yee, D. E. McNally, J. W. Simonson, S. Zellman, M. Klemm, P. Kamenov, G. Geschwind, A. Zebro, S. Ghose, J. Bai, E. Dooryhee, G. Kotliar, and M. C. Aronson, Proc. Natl. Acad. Sci. (2018), 10.1073/pnas. 1800284115.

137 W. Ruan, C. Hu, J. Zhao, P. Cai, Y. Peng, C. Ye, R. Yu, X. Li, Z. Hao, C. Jin, X. Zhou, Z.-Y. Weng, and Y. Wang, Science Bulletin 61, 1826 (2016). 
138 R. Molinder, T. P. Comyn, N. Hondow, J. E. Parkerc, and V. Duponta, Energy Environ. Sci. 5, 8958 (2012).

139 D. P. Shoemaker, Y.-J. Hu, D. Y. Chung, G. J. Halder, P. J. Chupas, L. Soderholm, J. F. Mitchell, and M. G. Kanatzidis, Proc. Natl. Acad. Sci. USA 111, 10922 (2014).

140 C.-H. Yee, T. Birol, and G. Kotliar, EPL 111, 17002 (2015).

141 S. P. Ong, L. Wang, B. Kang, and G. Ceder, Chemistry of Materials 20, 1798 (2008).

142 M. De Raychaudhury, E. Pavarini, and O. K. Andersen, Phys. Rev. Lett. 99, 126402 (2007).

143 C. E. Matt, D. Sutter, A. M. Cook, Y. Sassa, M. Mansson, O. Tjernberg, L. Das, M. Horio, D. Destraz, C. G. Fatuzzo, K. Hauser, M. Shi, M. Kobayashi, V. Strocov, P. Dudin, M. Hoesch, S. Pyon, T. Takayama, H. Takagi, O. J. Lipscombe, S. M. Hayden, T. Kurosawa, N. Momono, M. Oda, T. Neupert, and J. Chang, Nature Communications 9, 1 (2018), arXiv:1707.08491.

144 J. A. Slezak, J. Lee, M. Wang, K. McElroy, K. Fujita, B. M. Andersen, P. J. Hirschfeld, H. Eisaki, S. Uchida, and J. C. Davis, Proc. Natl. Acad. Sci. 105, 3203 (2008).

145 C. Weber, K. Haule, and G. Kotliar, Phys. Rev. B 82, 125107 (2010).

146 L. Li, X. Deng, Z. Wang, Y. Liu, M. Abeykoon, E. Dooryhee, A. Tomic, Y. Huang, J. B. Warren, E. S. Bozin, S. J. L. Billinge, Y. Sun, Y. Zhu, G. Kotliar, and C. Petrovic, npj Quantum Mater. 2, 11 (2017).

147 W. E. Pickett, Physica B: Condens. Matter. 296, 112 (2001).

148 A. Sleight, J. Gillson, and P. Bierstedt, Solid State Communications 17, 27 (1975).

149 R. J. Cava, B. Batlogg, J. J. Krajewski, R. Farrow, L. W. Rupp, A. E. White, K. Short, W. F. Peck, and T. Kometani, Nature 332, 814 (1988).

150 V. Meregalli and S. Y. Savrasov, Phys. Rev. B 57, 14453 (1998).

151 R. Nourafkan, F. Marsiglio, and G. Kotliar, Phys. Rev. Lett. 109, 017001 (2012).

152 M. Retuerto, T. Emge, J. Hadermann, P. W. Stephens, M. R. Li, Z. P. Yin, M. Croft, A. Ignatov, S. J. Zhang, Z. Yuan, C. Jin, J. W. Simonson, M. C. Aronson, A. Pan, D. N. Basov, G. Kotliar, and M. Greenblatt, Chemistry of Materials 25, 4071 (2013).

153 Z. P. Yin and G. Kotliar, EPL 101, 27002 (2013).

154 S. P. Ong, A. Jain, G. Hautier, B. Kang, and G. Ceder, Electrochemistry Communications 12, 427 (2010).

155 S. P. Ong, W. D. Richards, A. Jain, G. Hautier, M. Kocher, S. Cholia, D. Gunter, V. L. Chevrier, K. A. Persson, and G. Ceder, Computational Materials Science 68, 314 (2013)

156 M. Retuerto, Z. Yin, T. J. Emge, P. W. Stephens, M.-r. Li, T. Sarkar, M. C. Croft, A. Ignatov, Z. Yuan, S. J. Zhang, C. Jin, R. Paria Sena, J. Hadermann, G. Kotliar, and M. Greenblatt, Inorganic Chemistry 54, 1066 (2015).

157 A. Zunger, Appl. Phys. Lett. 83, 57 (2003).

158 S. Jiang, C. Liu, H. Cao, T. Birol, J. M. Allred, W. Tian, L. Liu, K. Cho, M. J. Krogstad, J. Ma, K. M. Taddei, M. A. Tanatar, M. Hoesch, R. Prozorov, S. Rosenkranz, Y. J. Uemura, G. Kotliar, and N. Ni, Phys. Rev. B 93, 054522 (2016).

159 Y. Kamihara, T. Watanabe, M. Hirano, and H. Hosono, J. Am. Chem. Soc. 130, 3296 (2008).
160 I. I. Mazin, D. J. Singh, M. D. Johannes, and M. H. Du, Phys. Rev. Lett. 101, 057003 (2008).

161 K. Kuroki, S. Onari, R. Arita, H. Usui, Y. Tanaka, H. Kontani, and H. Aoki, Phys. Rev. Lett. 101, 087004 (2008).

162 S. Graser, G. R. Boyd, C. Cao, H.-P. Cheng, P. J. Hirschfeld, and D. J. Scalapino, Phys. Rev. B 77, 180514(R) (2008).

163 A. V. Chubukov, D. V. Efremov, and I. Eremin, Phys. Rev. B 78, 134512 (2008).

164 M. Berciu, I. Elfimov, and G. A. Sawatzky, Phys. Rev. B 79, 214507 (2009).

165 S. Onari and H. Kontani, Phys. Rev. Lett. 103, 177001 (2009).

166 J. H. Shim, K. Haule, and G. Kotliar, Phys. Rev. B 79, 060501(R) (2009).

167 J. K. Wang, L. L. Zhao, Q. Yin, G. Kotliar, M. S. Kim, M. C. Aronson, and E. Morosan, Phys. Rev. B 84, 064428 (2011).

168 J. Park, G. Lee, F. Wolff-Fabris, Y. Y. Koh, E. J. Eom, Y. K. Kim, M. A. Farhan, Y. J. Jo, C. Kim, J. H. Shim, and J. S. Kim, Phys. Rev. Lett. 107, 126402 (2011).

169 G. Lee, M. A. Farhan, J. S. Kim, and J. H. Shim, Phys. Rev. B 87, 245104 (2013).

${ }^{170}$ Y. J. Jo, J. Park, G. Lee, M. J. Eom, E. S. Choi, J. H. Shim, W. Kang, and J. S. Kim, Phys. Rev. Lett. 113, 156602 (2014).

171 L.-L. Jia, Z.-H. Liu, Y.-P. Cai, T. Qian, X.-P. Wang, H. Miao, P. Richard, Y.-G. Zhao, Y. Li, D.-M. Wang, J.-B. He, M. Shi, G.-F. Chen, H. Ding, and S.-C. Wang, Phys. Rev. B 90, 035133 (2014).

172 Y. Feng, Z. Wang, C. Chen, Y. Shi, Z. Xie, H. Yi, A. Liang, S. He, J. He, Y. Peng, X. Liu, Y. Liu, L. Zhao, G. Liu, X. Dong, J. Zhang, C. Chen, Z. Xu, X. Dai, Z. Fang, and X. J. Zhou, Sci. Rep. 4, 5385 (2014).

173 K. Wang and C. Petrovic, Phys. Rev. B 86, 155213 (2012).

174 K. Wang, D. Graf, and C. Petrovic, Phys. Rev. B 87, 235101 (2013).

175 X. Shi, P. Richard, K. Wang, M. Liu, C. E. Matt, N. Xu, R. S. Dhaka, Z. Ristic, T. Qian, Y.-F. Yang, C. Petrovic, M. Shi, and H. Ding, Phys. Rev. B 93, 081105(R) (2016).

176 N. Katayama, K. Kudo, S. Onari, T. Mizukami, K. Sugawara, Y. Kitahama, K. Iba, K. Fujimura, N. Nishimoto, M. Nohara, and H. Sawa, J. Phys. Soc. Jpn. 82, 123702 (2013).

177 H. Yakita, H. Ogino, T. Okada, A. Yamamoto, K. Kishito, T. Tohei, Y. Ikuhara, Y. Gotoh, H. Fujihisa, K. Kataoka, H. Eisaki, and J. ichi Shimoyama, J. Am. Chem. Soc. 136, 846 (2014).

178 C.-J. Kang, T. Birol, and G. Kotliar, Phys. Rev. B 95, 014511 (2017).

179 J. W. Harter, H. Chu, S. Jiang, N. Ni, and D. Hsieh, Phys. Rev. B 93, 104506 (2016).

180 Y. Mizuguchi, Y. Hara, K. Deguchi, S. Tsuda, T. Yamaguchi, K. Takeda, H. Kotegawa, H. Tou, and Y. Takano, Supercond. Sci. Technol. 23, 054013 (2010).

181 X. Wu, C. Le, Y. Liang, S. Qin, H. Fan, and J. Hu, Phys. Rev. B 89, 205102 (2014).

182 X. Wu, S. Qin, Y. Liang, C. Le, H. Fan, and J. Hu, Phys. Rev. B 91, 081111(R) (2015).

183 Y. Yasui, H. Sasaki, M. Sato, M. Ohashi, Y. Sekine, C. Murayama, and N. Môri, J. Phys. Soc. Jpn. 68, 1313 (1999). 
184 E. J. T. Salter, J. N. Blandy, and S. J. Clarke, Inorg. Chem. 55, 1697 (2016).

185 V. M. Zainullina and M. a. Korotin, Phys. Solid State 53, 978 (2011).

186 V. M. Zainullina, N. A. Skorikov, and M. A. Korotin, Phys. Solid State 54, 1864 (2012).

187 P. Blaha, K. Schwarz, G. K. H. Madsen, D. Kavasnicka, and J. Luitz, Wien2k (Karlheinz Schwarz, Tech. Universität Wien, Vienna, 2001).

188 S. Qin, Y. Li, Q. Zhang, C. Le, and J. Hu, Frontiers in Physics 13 (2018), https://doi.org/10.1007/s11467-0180745-7, arXiv:1702.08304.

189 H. He and M. Aaronson, "private communication," (2017).

190 C.-J. Kang and G. Kotliar, Phys. Rev. Materials 2, 034604 (2018).

191 M. Shishkin, M. Marsman, and G. Kresse, Phys. Rev. Lett. 99, 14 (2007).

192 A. L. Kutepov, Phys. Rev. B 95, 195120 (2017).

193 C. Franchini, A. Sanna, M. Marsman, and G. Kresse, Phys. Rev. B 81, 085213 (2010).

194 W. Kang and M. S. Hybertsen, Phys. Rev. B 82, 195108 (2010).

195 C. H. P. Wen, H. C. Xu, Q. Yao, R. Peng, X. H. Niu, Q. Y. Chen, Z. T. Liu, D. W. Shen, Q. Song, X. Lou, Y. F. Fang, X. S. Liu, Y. H. Song, Y. J. Jiao, T. F. Duan, H. H. Wen, P. Dudin, G. Kotliar, Z. P. Yin, and D. L. Feng, Physical Review Letters 121 (2018), https://doi.org/10.1103/PhysRevLett.121.117002, arXiv:1802.10507.

196 B. Fultz, Prog. Mater. Sci. 55, 247 (2010).

197 K. Haule and T. Birol, Phys. Rev. Lett. 115, 256402 (2015).
198 K. Haule and G. L. Pascut, Physical Review B 94, 195146 (2016), arXiv:1602.02819.

199 X. Deng, L. Wang, X. Dai, and Z. Fang, Phys. Rev. B 79, $075114(2009)$.

200 Y. X. Yao, J. Schmalian, C. Z. Wang, K. M. Ho, and G. Kotliar, Phys. Rev. B 84, 245112 (2011).

201 N. Lanata, X. Deng, and G. Kotliar, Phys. Rev. B 92 , 081108(R) (2015).

202 W.-S. Wang, X.-M. He, D. Wang, Q.-H. Wang, Z. D. Wang, and F. C. Zhang, Phys. Rev. B 82, 125105 (2010).

203 M. Sandri, M. Capone, and M. Fabrizio, Phys. Rev. B 87, 205108 (2013).

204 P. Liu, M. Kaltak, J. Klimeš, and G. Kresse, Phys. Rev. B 94, 165109 (2016).

205 H. Peng and S. Lany, Phys. Rev. B 87, 174113 (2013).

206 J. A. Schiller, L. K. Wagner, and E. Ertekin, Phys. Rev. B 92, 235209 (2015).

207 T.-H. Lee, Y.-X. Yao, V. Stevanović, V. Dobrosavljević, and N. Lanatà, arXiv:1710.08586 (2017).

208 E. B. Isaacs and C. Wolverton, Phys. Rev. Materials 2, 063801 (2018).

209 J. Harl and G. Kresse, Phys. Rev. Lett. 103, 056401 (2009).

210 "Materials genome initiative: https://www.mgi.gov/," .

211 D. G. Schlom, L.-Q. Chen, X. Pan, A. Schmehl, and M. A. Zurbuchen, Journal of the American Ceramic Society 91, 2429 (2008).

212 "The nomad repository http://nomad-repository.eu/," .

213 Y. Li, J. Hao, H. Liu, Y. Li, and Y. Ma, J. Chem. Phys. 140, 174712 (2014).

214 S. Lany, Phys. Rev. B 78, 245207 (2008).

215 L. Wang, T. Maxisch, and G. Ceder, Phys. Rev. B 73, 195107 (2006).

216 A. Jain, G. Hautier, S. P. Ong, C. J. Moore, C. C. Fischer, K. A. Persson, and G. Ceder, Phys. Rev. B 84, 045115 (2011). 